\title{
Neutrino flux prediction at MiniBooNE
}

A. A. Aguilar-Arevalo, ${ }^{5}$ C. E. Anderson, ${ }^{16}$ A. O. Bazarko, ${ }^{13}$ S. J. Brice,${ }^{7}$ B. C. Brown, ${ }^{7}$ L. Bugel,${ }^{5}$ J. Cao, ${ }^{12, *}$ L. Coney,${ }^{5,}$ J. M. Conrad, ${ }^{5}$ D. C. Cox, ${ }^{9}$ A. Curioni, ${ }^{16}$ Z. Djurcic, ${ }^{5}$ D. A. Finley, ${ }^{7}$ B. T. Fleming,${ }^{7,16}$ R. Ford, ${ }^{7}$ F. G. Garcia, ${ }^{7}$ G. T. Garvey, ${ }^{10}$ C. Green, ${ }^{7,10}$ J. A. Green, ${ }^{9,10}$ T. L. Hart, ${ }^{4,}$ E. Hawker,,${ }^{3,10,8}$ R. Imlay, ${ }^{11}$ R. A. Johnson, ${ }^{3}$ G. Karagiorgi, ${ }^{5}$ P. Kasper, ${ }^{7}$ T. Katori, ${ }^{9}$ T. Kobilarcik, ${ }^{7}$ I. Kourbanis, ${ }^{7}$ S. Koutsoliotas, ${ }^{2}$ E. M. Laird, ${ }^{13}$ S. K. Linden, ${ }^{16}$ J. M. Link, ${ }^{5,15}$ Y. Liu, ${ }^{12}$ Y. Liu, ${ }^{1}$ W. C. Louis, ${ }^{10}$ K. B. M. Mahn, ${ }^{5}$ W. Marsh, ${ }^{7}$ P. S. Martin, ${ }^{7}$ G. McGregor, ${ }^{10}$ W. Metcalf, ${ }^{11}$ P. D. Meyers, ${ }^{13}$ F. Mills, ${ }^{7}$ G. B. Mills,${ }^{10}$ J. Monroe,${ }^{5, l}$ C. D. Moore,${ }^{7}$ R. H. Nelson, ${ }^{4}$ V. T. Nguyen, ${ }^{5}$ P. Nienaber,${ }^{14}$ J. A. Nowak, ${ }^{11}$ S. Ouedraogo, ${ }^{11}$ R. B. Patterson, ${ }^{13, \pi}$ D. Perevalov, ${ }^{1}$ C. C. Polly, ${ }^{9}$ E. Prebys, ${ }^{7}$ J. L. Raaf, ${ }^{3, * *}$ H. Ray, ${ }^{8,10}$ B. P. Roe, ${ }^{12}$ A. D. Russell, ${ }^{7}$ V. Sandberg, ${ }^{10}$ R. Schirato, ${ }^{10}$ D. Schmitz, ${ }^{5}$ M. H. Shaevitz,${ }^{5}$ F. C. Shoemaker, ${ }^{13}$ D. Smith, ${ }^{6}$ M. Soderberg, ${ }^{16}$ M. Sorel, ${ }^{5, \dagger}$ P. Spentzouris, ${ }^{7}$ I. Stancu, ${ }^{1}$ R. J. Stefanski, ${ }^{7}$ M. Sung, ${ }^{11}$ H. A. Tanaka, ${ }^{13,+*}$ R. Tayloe, ${ }^{9}$ M. Tzanov, ${ }^{4}$ R. Van de Water, ${ }^{10}$ M. O. Wascko, ${ }^{11, \S}$ D. H. White, ${ }^{10}$ M. J. Wilking, ${ }^{4}$ H. J. Yang, ${ }^{12}$ G. P. Zeller, ${ }^{5,10}$ and E. D. Zimmerman ${ }^{4}$

\section{(MiniBooNE Collaboration)}

\author{
${ }^{1}$ University of Alabama; Tuscaloosa, Alabama 35487, USA \\ ${ }^{2}$ Bucknell University; Lewisburg, Pennsylvania 17837, USA \\ ${ }^{3}$ University of Cincinnati; Cincinnati, Ohio 45221, USA \\ ${ }^{4}$ University of Colorado; Boulder, Colorado 80309, USA \\ ${ }^{5}$ Columbia University; New York, New York 10027, USA \\ ${ }^{6}$ Embry-Riddle Aeronautical University; Prescott, Arizona 86301, USA \\ ${ }^{7}$ Fermi National Accelerator Laboratory; Batavia, Illinois 60510, USA \\ ${ }^{8}$ University of Florida; Gainesville, Florida 32611, USA \\ ${ }^{9}$ Indiana University; Bloomington, Indiana 47405, USA \\ ${ }^{10}$ Los Alamos National Laboratory; Los Alamos, New Mexico 87545, USA \\ ${ }^{11}$ Louisiana State University; Baton Rouge, Louisiana 70803, USA \\ ${ }^{12}$ University of Michigan; Ann Arbor, Michigan 48109, USA \\ ${ }^{13}$ Princeton University; Princeton, New Jersey 08544, USA \\ ${ }^{14}$ Saint Mary's University of Minnesota; Winona, Minnesota 55987, USA \\ ${ }^{15}$ Virginia Polytechnic Institute \& State University; Blacksburg, Virginia 24061, USA \\ ${ }^{16}$ Yale University; New Haven, Connecticut 06520, USA \\ (Received 21 June 2008; published 15 April 2009)
}

\begin{abstract}
The booster neutrino experiment (MiniBooNE) searches for $\nu_{\mu} \rightarrow \nu_{e}$ oscillations using the $\mathcal{O}(1 \mathrm{GeV})$ neutrino beam produced by the booster synchrotron at the Fermi National Accelerator Laboratory). The booster delivers protons with $8 \mathrm{GeV}$ kinetic energy $(8.89 \mathrm{GeV} / c$ momentum) to a beryllium target, producing neutrinos from the decay of secondary particles in the beam line. We describe the Monte Carlo simulation methods used to estimate the flux of neutrinos from the beam line incident on the MiniBooNE detector for both polarities of the focusing horn. The simulation uses the Geant 4 framework for propagating particles, accounting for electromagnetic processes and hadronic interactions in the beam line materials, as well as the decay of particles. The absolute double differential cross sections of pion and kaon production in the simulation have been tuned to match external measurements, as have the hadronic cross sections for nucleons and pions. The statistical precision of the flux predictions is enhanced through reweighting and resampling techniques. Systematic errors in the flux estimation have been determined by varying parameters within their uncertainties, accounting for correlations where appropriate.
\end{abstract}

\footnotetext{
*Present Address: Institute of High-Energy Physics; Beijing 100049, China

${ }^{\dagger}$ Present Address: Hope College; Holland, MI 49423, USA

${ }^{\ddagger}$ Present Address: Illinois Institute of Technology; Chicago, IL 60616, USA

${ }^{\S}$ Present Address: Illinois Mathematics and Science Academy; Aurora IL 60506, USA

"Present Address: Massachusetts Institute of Technology; Cambridge, MA 02139, USA

IIPresent Address: California Institute of Technology; Pasadena, CA 91125, USA

**Present Address: Boston University; Boston, MA 02215, USA

${ }^{\dagger}$ Present Address: IFIC, Universidad de Valencia and CSIC; 46071 Valencia, Spain

${ }^{\ddagger}$ Present Address: University of British Columbia, Vancouver, BC V6T 1Z1, Canada

${ }^{\S}$ Present Address: Imperial College; London SW7 2AZ, United Kingdom, USA
} 


\section{INTRODUCTION}

The booster neutrino experiment (MiniBooNE) at Fermilab searches for the oscillation of muon neutrinos $\left(\nu_{\mu}\right)$ to electron neutrinos $\left(\nu_{e}\right)$ indicated by the LSND experiment $[1,2]$. The neutrino beam is produced by the booster neutrino beam line (BNB), where protons with $8 \mathrm{GeV}$ kinetic energy $(8.89 \mathrm{GeV} / c$ momentum) are extracted from the Fermilab booster synchrotron and directed towards a beryllium target. Secondary mesons produced by the interaction of the protons on the target decay to produce a neutrino beam with an average energy of $\sim 800 \mathrm{MeV}$. Neutrino interactions are observed in a 6.1-meter-radius, spherical detector situated 541 meters from the center of the target. The detector is composed of 800 metric tons of mineral oil that serves as both the target for neutrino interactions and the medium in which charged particles produced in neutrino interactions radiate Cherenkov and scintillation photons. The photons are detected on an array of 1520 photomultipliers, and the resulting spatial and temporal patterns of light are used to identify and reconstruct the interactions. Understanding both the spectrum and composition of the neutrino beam is critical to the neutrino oscillation analysis, which searches for an excess of $\nu_{e}$ events over a background of both nonoscillation sources of $\nu_{e}$ in the beam line and misidentified $\nu_{\mu}$ interactions.

The neutrino oscillation analysis at MiniBooNE utilizes observed data to constrain the uncertainties in the expected event rates of certain key processes. These constraints typically reduce the uncertainties that would result from a direct estimation using solely the predicted neutrino flux and cross sections. Within the $\nu_{\mu} \rightarrow \nu_{e}$ oscillation analysis, the observed rate of $\nu_{\mu}$ charged-current quasielastic events and neutral current $\pi^{0}$ events are used directly in the estimation of the number and spectrum of background and expected neutrino oscillation events. The Monte Carlobased flux prediction described here is one input to this process. In this article, we focus on the flux prediction itself, which is based on external data, without regard to the observed neutrino rates at MiniBooNE. Predictions for both polarities of the focusing horn are presented. Detailed comparisons of the observed and predicted neutrino event rates, as well as descriptions of the use of the predicted neutrino fluxes in various analyses, are described in publications relating to the analysis of the neutrino data itself, e.g., Refs. [2-5].

\section{THE BOOSTER NEUTRINO BEAM LINE}

The booster neutrino beam line (BNB) produces neutrinos using $8.89 \mathrm{GeV} / c$ momentum protons from the boos- ter synchrotron that are incident on a beryllium target. The layout of the BNB is shown in Fig. 1. The target is embedded within a pulsed electromagnet (the "horn") that produces a toroidal magnetic field to focus positive secondary particles and defocus negative secondary particles emerging from proton-beryllium interactions. These secondary particles enter a 50-meter-long decay region, resulting in a neutrino-enhanced beam. The polarity of the horn can be reversed to focus negative secondary particles and produce an antineutrino-enhanced beam. The axis of the beam, defined by the center of the decay pipe, is displaced vertically from the center of the MiniBooNE detector by 1.9 meters.

The particle production is dominated by pions, though there is significant kaon production as well. Neutrinos also result from the decay of muons whose primary source is the decay of pions produced in the target. This results in a significant flux of $\nu_{e} / \bar{\nu}_{e}$ in neutrino/antineutrino mode, while the corresponding flux of $\bar{\nu}_{\mu} / \nu_{\mu}$ is small compared to the $\nu_{\mu} / \bar{\nu}_{\mu}$ which result directly from the decay of the pions. A beam stop at the end of the decay region absorbs particles apart from the neutrinos. The predicted composition of the neutrino beam is described in Sec. VI. A detailed description of the BNB can be found in the Technical Design Report for the BNB [6]. This section describes the beam line geometry and components relevant for the neutrino flux prediction.

\section{A. FNAL Booster and Proton extraction}

The Fermi National Accelerator Laboratory (FNAL) booster is a 474-meter-circumference synchrotron operating at $15 \mathrm{~Hz}$. Protons from the Fermilab LINAC are injected at $400 \mathrm{MeV}$ and accelerated to $8 \mathrm{GeV}$ kinetic energy $(8.89 \mathrm{GeV} / c$ momentum). The booster has a harmonic number of 84 , of which 81 buckets are filled. The beam is extracted into the BNB using a fast-rising kicker that extracts all of the particles in a single turn. The resulting structure is a series of 81 bunches of protons each $\sim 2$ ns wide and 19 ns apart.

Upon leaving the booster, the proton beam is transported through a lattice of focusing and defocusing quadrupole (FODO) and dipole magnets. A switch magnet steers the beam to the main injector or to the BNB. The BNB is also a FODO that terminates with a triplet that focuses the beam on the target. The design and measured optics of BNB are in agreement $[7,8]$.

The maximum allowable average repetition rate for delivery of protons to the $\mathrm{BNB}$ is $5 \mathrm{~Hz}$ (with a maximum of 11 pulses in a row at $15 \mathrm{~Hz}$ ) and $5 \times 10^{12}$ protons-perpulse. The $5 \mathrm{~Hz}$ limit is set by the design of the horn (described below) and its power supply. As of January 


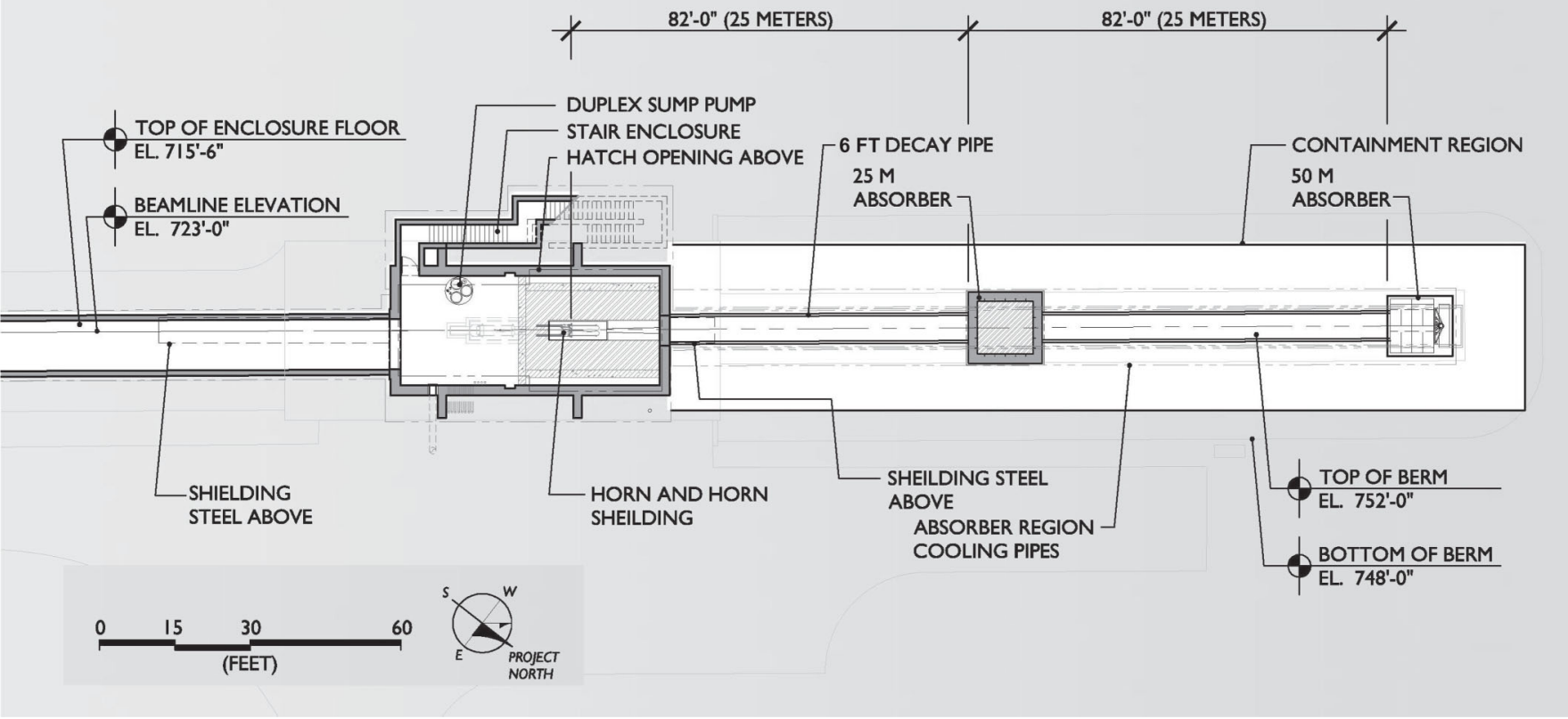

FIG. 1 (color online). Overall layout of the BNB. The primary proton beam, extracted from the booster, enters the target hall from the left. Upon exiting the target hall, particles encounter a 50-meter-long decay region, terminating in the beam stop on the right.

2008, over $10^{21}$ protons have been delivered to the BNB, with a typical up time of greater than $90 \%$ during normal operations. The neutrino oscillation results in neutrino mode were published using $5.6 \times 10^{20}$ protons-on-target delivered prior to 2006 , when the polarity of the horn was reversed to collect antineutrino mode data [9].

\section{B. Target}

The target consists of seven identical cylindrical slugs of beryllium arranged to produce a cylinder $71.1 \mathrm{~cm}$ long and $0.51 \mathrm{~cm}$ in radius. The target is contained within a beryllium sleeve $0.9 \mathrm{~cm}$ thick with an inner radius of $1.37 \mathrm{~cm}$. Each target slug is supported within the sleeve by three "fins" (also beryllium) which extend radially out from the target to the sleeve. The volume of air within the sleeve is circulated to provide cooling for the target when the beam line is in operation. The target and associated assembly are shown in Fig. 2, where the top figure shows an "exploded" view of the various components (with the downstream end of the target on the right), and the bottom shows the components in assembled form. The choice of beryllium as the target material was motivated by residual radioactivity issues in the event that the target assembly needed to be replaced, as well as energy loss considerations that allow the air-cooling system to be sufficient.

Upstream of the target, the primary proton beam is monitored using four systems: two toroids measuring its intensity (protons-per-pulse), beam position monitors (BPM) and a multiwire chamber determining the beam width and position, and a resistive wall monitor (RWM) measuring both the time and intensity of the beam spills. The vacuum of the beam pipe extends to about 5 feet upstream of the target, minimizing upstream proton interactions.

The toroids are continuously calibrated at $5 \mathrm{~Hz}$ with their absolute calibrations verified twice a year. The calibrations have shown minimal deviation $(<0.5 \%)$. The proton flux measured in the two toroids agree to within $2 \%$, compatible with the expected systematic uncertainties. The BPMs are split-plate devices that measure the difference of charge induced on two plates. By measuring the change in beam position at several locations without intervening optics, the BPMs are found to be accurate to $0.1 \mathrm{~mm}$ (standard deviation). The multiwire is a wire chamber with 48 horizontal and 48 vertical wires and $0.5 \mathrm{~mm}$ pitch. The profile of the beam is measured using the secondary emission induced by the beam on the wires.

The RWM is located upstream of the target to monitor the time and intensity of the proton pulses prior to striking the target. While the data from the RWM did not directly enter the $\nu_{\mu} \rightarrow \nu_{e}$ analysis, it allowed many useful cross checks, such as those shown in Fig. 3. The left figure shows a comparison of the production times of neutrinos observed in the MiniBooNE detector estimated based on the vertex and time reconstructed by the detector and subtracting the time-of-flight. This time is then compared to the nearest bucket as measured by the RWM. The distribution indicates that neutrino events can be matched not only to pulses from the booster, but to a specific bucket within the pulse. The tails of the distribution result from the resolution of the 

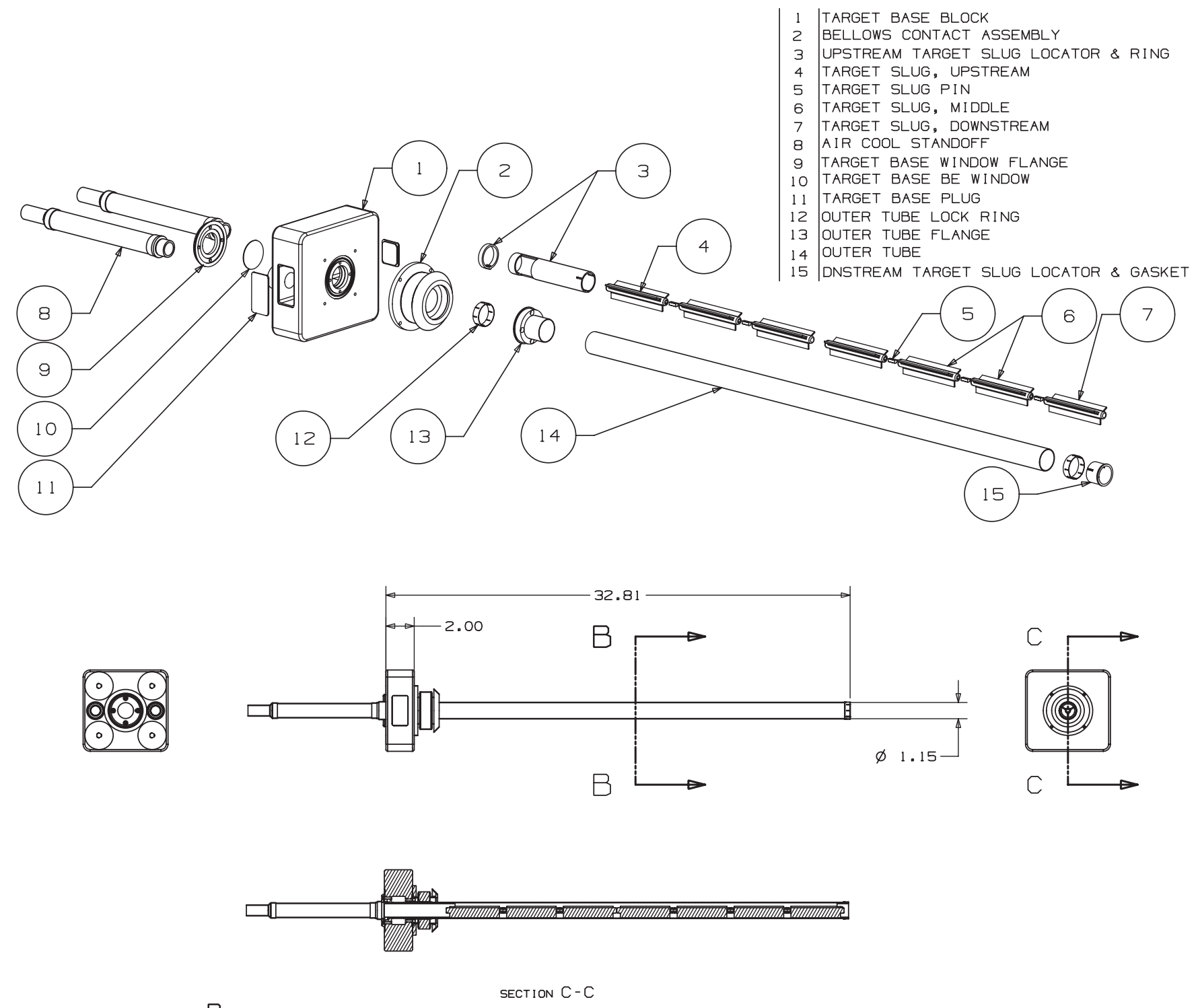

SECTION B

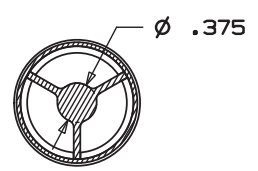

FIG. 2. The MiniBooNE target assembly. The top shows an exploded view of the components, while the bottom shows the assembled configuration. The proton beam enters from the left in both figures, striking the finned beryllium slugs. Dimensions are in inches.

vertex reconstruction of the neutrino event in the detector, which is needed to determine the time of the event and correct for the time-of-flight. The right plot demonstrates the synchronization of the horn pulse (described in Sec. IIC) with the delivery of the beam as measured by the RWM.

\section{Horn electromagnet}

The horn, shown in Fig. 4, is a pulsed toroidal electromagnet composed of an aluminum alloy (6061 T6). The current in the horn is a $143 \mu$ s-long pulse with a nominal peak of $170 \mathrm{kA}$ coinciding with the arrival of the proton beam at the target. The actual operating values are typically $174 \mathrm{kA}$ in both neutrino and antineutrino mode with $\sim 1 \mathrm{kA}$ variations. In neutrino mode, the flow of current (in the positive sense) runs along the inner conductor (containing the target), which folds outwards at a length of $185 \mathrm{~cm}$ to return via the outer conducting cylinder of the horn at $30 \mathrm{~cm}$ radius. Within the horn cavity, defined by the volume between the outer and inner conducting cylinders, the pulse creates a magnetic field that falls as $1 / R$, where $R$ is the distance from the cylindrical symmetry axis of the 

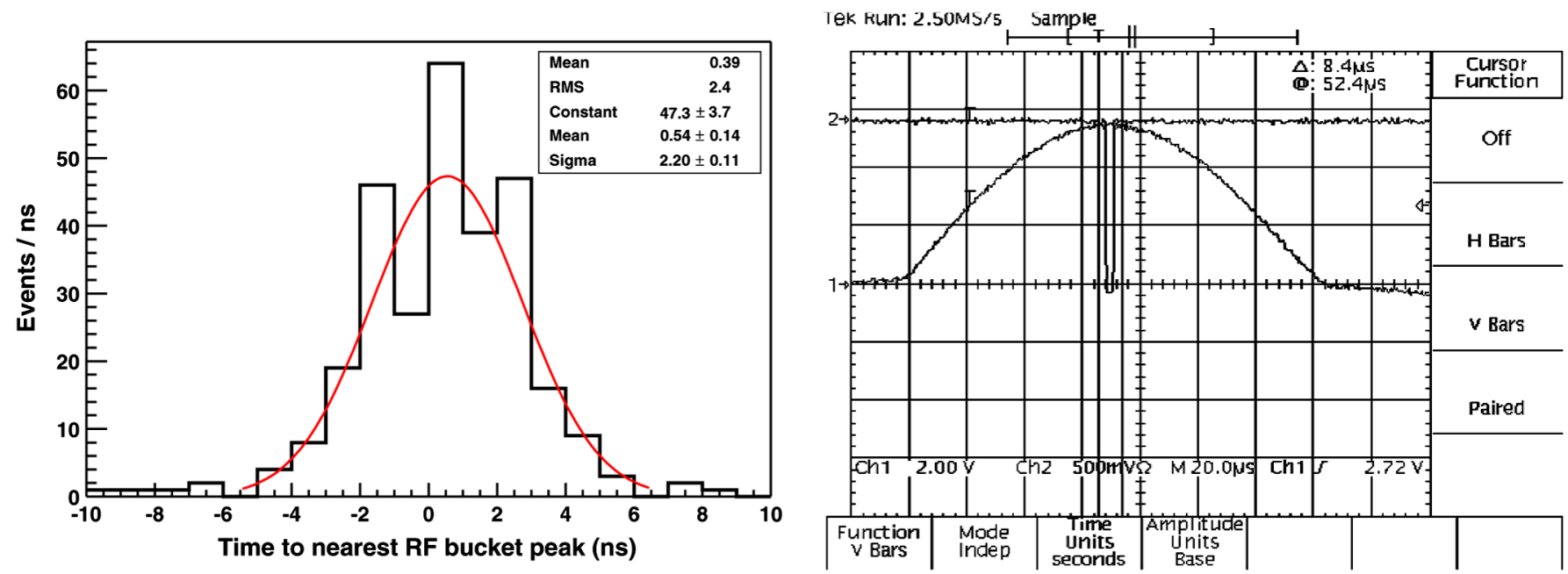

FIG. 3 (color online). Left: Neutrino event times relative to the nearest RF bucket (measured by the RWM) corrected for expected time-of-flight. Right: An oscilloscope trace showing the coincidence of the beam delivery with the horn pulse. The top trace (labeled " 2 " on the left) is a discriminated signal from the resistive wall monitor (RWM), indicating the arrival of the beam pulse. The bottom trace (labeled " 1 " on the left) is the horn pulse. The horizontal divisions are $20 \mu$ s each.

horn. The largest field values of 1.5 Tesla are obtained where the inner conductor is narrowest $(2.2 \mathrm{~cm}$ radius). The effects of time-varying fields within the cavity of the horn are found to be negligible. The expected field properties of the horn have been verified by measuring the current induced in a wire coil inserted into the portals of the horn. Figure 5 shows the measured $R$ dependence of the azimuthal magnetic field compared with the expected $1 / R$ dependence. The "skin effect", in which the time-varying currents traveling on the surface of the conductor penetrate into the conductor, results in electromagnetic fields within the conductor itself.

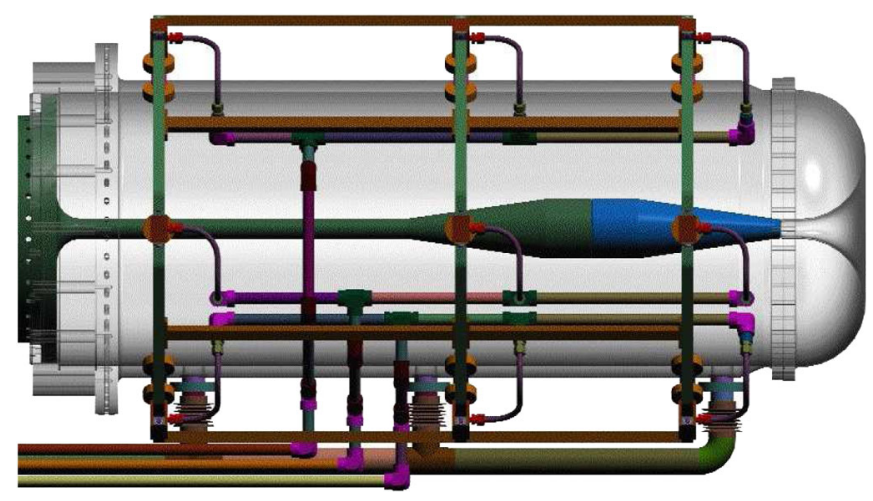

FIG. 4 (color online). The MiniBooNE pulsed horn system. The outer conductor (gray) is transparent to show the inner conductor components running along the center (dark green and blue). The target assembly is inserted into the inner conductor from the left side. In neutrino-focusing mode, the (positive) current flows from left-to-right along the inner conductor, returning along the outer conductor. The plumbing associated with the water cooling system is also shown.
During operation, the horn is cooled by a closed water system which sprays water onto the inner conductor via portholes in the outer cylinder. The target assembly is rigidly fixed to the upstream face of the horn, although the target is electrically isolated from its current path. At the time of writing, two horns have been in operation in the BNB. The first operated for $96 \times 10^{6}$ pulses before failing,

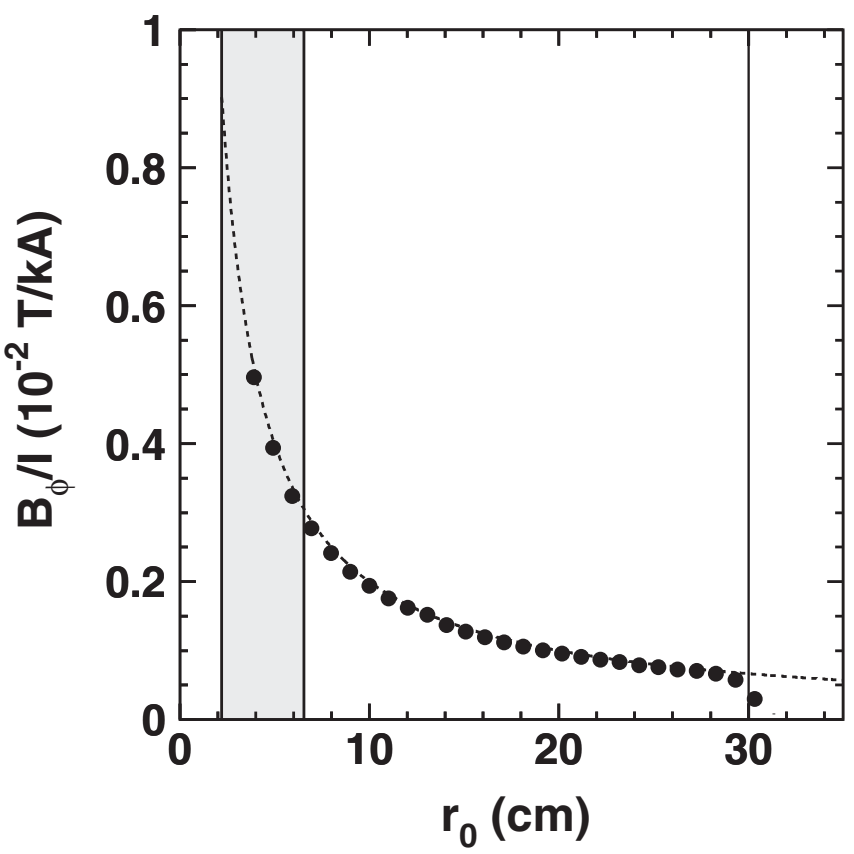

FIG. 5. Measurements of the azimuthal magnetic field within the horn. The points show the measured magnetic field, while the line shows the expected $1 / R$ dependence. The black lines indicate the minimum and maximum radii of the inner conductor. 
while the second is still in operation as of this writing after over $130 \times 10^{6}$ pulses [10].

\section{Collimator}

A concrete collimator is located downstream of the target/horn assembly and serves as the entrance into the decay region. The collimator absorbs particles that would not otherwise contribute to the neutrino flux and is $214 \mathrm{~cm}$ long, with an upstream aperture of $30 \mathrm{~cm}$ radius that grows to $35.5 \mathrm{~cm}$ on the downstream end. By absorbing these particles, the collimator reduces radiation elsewhere in the beam line. The upstream end of the collimator is located $259 \mathrm{~cm}$ from the upstream face of the target. Simulations indicate that the collimator does not limit the neutrino flux at the MiniBooNE detector.

\section{E. Decay region and absorber}

The air-filled cylindrical decay region following the collimator is 3 feet in radius and extends for 45 meters, terminated by the beam stop 50 meters from the upstream face of the target. Survey data indicate that the constructed decay region is 49.87 meters, including the collimator region. The wall of the decay region is a corrugated steel pipe surrounded by packed dolomite gravel $\left(\mathrm{CaMg}\left(\mathrm{CO}_{2}\right)_{3}\right.$, $\left.\rho=2.24 \mathrm{~g} / \mathrm{cm}^{3}\right)$. The beam stop itself is made of steel and concrete, within which is an array of gas proportional counters that detects muons penetrating the beam stop.

To allow potential systematic studies, a set of ten steel absorbing plates are positioned above the decay pipe at 25 meters. When lowered into the decay region, the steel absorbers reduce the effective decay path from 50 to 25 meters. This has the effect of reducing the overall flux, but preferentially reducing the decay of the longerlived muons, a major source of nonoscillation $\nu_{e}$ background. The $25 \mathrm{~m}$ absorber was not deployed during the neutrino running for the $\nu_{\mu} \rightarrow \nu_{e}$ oscillation analysis.

\section{F. Little Muon Counter}

The Little Muon Counter (LMC) is an off-axis spectrometer that measures the rate and spectrum of muons produced at a $7^{\circ}$ angle to the beam axis in the decay pipe pointing back to the alcove for the $25 \mathrm{~m}$ absorber. The detector consists of a $40 \mathrm{ft}$ drift pipe extending from the decay region at $7^{\circ}$ leading to an enclosure. The kinematics of the two-body pion and kaon decay are such that kaons produce a momentum distribution peaked at $1.8 \mathrm{GeV} / c$ at this angle, whereas pions produce muons at lower momentum. Muons sent down the drift pipe to the enclosure encounter an iron collimator with a tungsten core that further restricts the angular acceptance of the counter and reduces backgrounds. Following the collimator, the muon momentum is determined by a spectrometer consisting of a dipole magnet and planes of scintillating fiber trackers. Finally, a range stack consisting of alternating scintillator and tungsten layers allow high-energy muons to be distinguished from pions and other particles based on the number of tungsten planes penetrated by the particle. Further details on the LMC can be found in Ref. [11].

\section{GEANT4-BASED MONTE CARLO SIMULATION}

The properties of the MiniBooNE neutrino flux are determined using a Geant4-based Monte Carlo simulation [12]. The simulation can be divided roughly into five steps:

(i) The definition of the beam line geometry, specified by the shape, location, and composition of the components of the $\mathrm{BNB}$, through which the primary protons and all other particles propagate (Sec. III A).

(ii) The generation of the primary protons according to the expected beam optics properties upstream of the target (Sec. III C).

(iii) The simulation of particles produced in the primary $p$-Be interactions, including the elastic and quasielastic scattering of protons in the target. Custom tables for the production of protons, neutrons, $\pi^{ \pm}$, $K^{ \pm}$and $K^{0}$ in these interactions have been developed to accommodate production models based on external data as described in Sec. III E.

(iv) The propagation of the particles using the Geant 4 framework, accounting for energy loss and electromagnetic and hadronic processes that alter the kinematics of the particles as described in Sec. IIID. Hadronic interactions and decay processes may also annihilate the particle in the tracking process and create new particles to be tracked. Within the horn, the effect of the expected magnetic field on the trajectory of the particles is accounted for (Sec. III B).

(v) The identification of decay processes that result in neutrinos. The simulation of the decays is handled by a custom decay model, described in Sec. III F, outside of the Geant 4 framework. The decay model reflects the latest branching fraction measurements and simulates polarization effects and kinematic distributions resulting from decay form factors. A number of techniques to enhance the statistical precision of the flux prediction are employed (Sec. III G).

\section{A. Geant4 description of the BNB geometry:}

The Geant 4 Monte Carlo geometry consists of the last 50 meters of the booster beam line, the target hall, and the $50 \mathrm{~m}$ meson decay volume. The geometry description is defined to match the actual constructed beam line as closely as possible; differences from the specifications are noted here. Since the generation of primary protons in the simulation starts immediately upstream of the target (see Sec. III C), the geometry description of the beam line leading to the target is simplified. Each section is simulated 


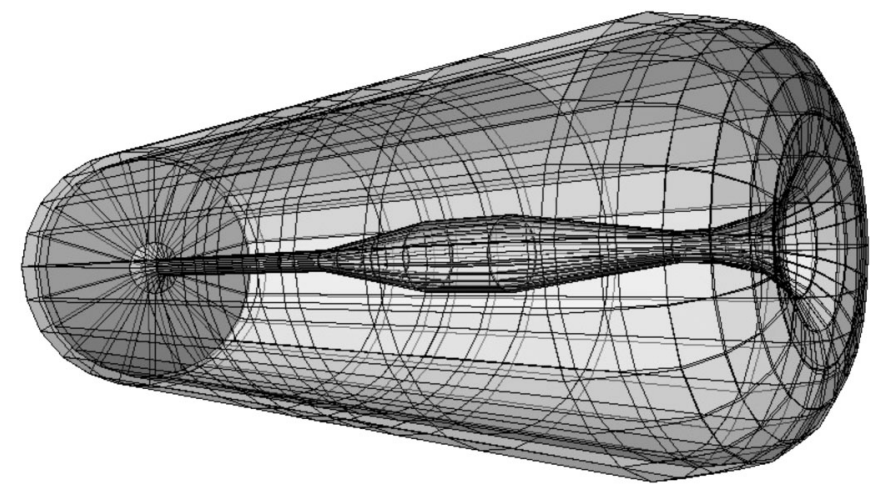

FIG. 6. The MiniBooNE horn as simulated in the Geant4 beam Monte Carlo.

with concrete walls surrounded by a uniform bed of dolomite. The entire structure is filled with air at standard temperature and pressure.

The simulated target hall contains the target, horn, and secondary beam collimator. In addition to the concrete walls, the target hall is lined with 1.28 meters of steel shielding. The seven slugs of the target and the target sleeve, together with the fins which support them within the sleeve, compose the simulated target geometry. The horn is constructed using an aluminum Geant polycone that specifies the inner and outer radius at 14 different points along the direction of the beam. A polycone of air is placed inside of the aluminum polycone to set the thickness of the inner and outer conductors. A graphical representation of the Monte Carlo horn is shown in Fig. 6.

The meson decay region is simulated as two 20-meterlong decay pipes separated by the $25 \mathrm{~m}$ absorber enclosure, followed by the beam stop. The decay pipes are made of concrete with an inner diameter of 6 feet and an outer diameter of 10 feet. In contrast, the actual decay pipe is corrugated steel and surrounded by dolomite; this simplification is not expected to affect the flux prediction.

\section{B. Horn magnetic field}

The $1 / R$ magnetic field expected from the current path within the horn is simulated in the volume corresponding to the cavity of the horn. The strength of the field corresponds to a $174 \mathrm{kA}$ current running along the inner conductor (reversed when simulating anti-neutrino mode). In addition, the permeation of the magnetic field into the inner conducting cylinder of the horn from the skin effect (described in Sec. VII) is included in the simulation. The predicted trajectories for charged particles in the magnetic field in the Geant 4 simulation have been checked in an external study using the DRKNYS routine from CERNLIB [13], an independently implemented numerical method.

\section{Generation of the primary proton beam}

The primary protons are simulated individually, since no correlated effects between the protons in a bunch are expected. The properties of the proton beam, such as the position and profile, have been simulated using TRANSPORT [14] and verified by upstream beam monitors [8]. The protons are generated $1 \mathrm{~cm}$ upstream of the target with the transverse $(x, y)$ positions drawn from random Gaussian distributions with $0 \mathrm{~mm}$ mean and $1.51 \mathrm{~mm}$ and $0.75 \mathrm{~mm}$ widths, respectively. Likewise, the angular deviations of the proton direction from the $z$ direction, $\theta_{x}$ and $\theta_{y}$, are drawn from Gaussian distributions with $0 \mathrm{mrad}$ mean and $0.66 \mathrm{mrad}$ and $0.40 \mathrm{mrad}$ width, respectively. The number of protons that undergo inelastic interactions in the target (as opposed to scattering out) is studied in Sec. VII A. In particular, while the default configuration describes a diverging beam, the TRANSPORT simulations indicate that the protons are expected to be convergent on the target, with a "waist" of zero divergence at the center of the target. The simulated beam configuration is such that $99.8 \%$ of the protons are on a trajectory to intersect the target. The studies in Sec. VII A indicate that reasonable perturbations to the model, including the expected focusing configuration, do not affect the predicted neutrino flux by more than $1 \%$.

\section{Particle tracking and propagation}

Particles, whether they are primary protons or particles produced in the simulation, are tracked and propagated within the Geant 4 framework with full accounting for electromagnetic and hadronic interactions and decays. Within each medium, the Coulomb scattering and energy loss are calculated in each step of the tracking, and the particle trajectory and energy updated accordingly. The energy loss and deflection angles predicted by the framework have been checked in a comparison with the BetheBloch formalism and the Highland formula [10]. The rate of hadronic interactions for protons, neutrons, and charged pions on beryllium and aluminum are governed by customized cross section tables (see Sec. IV) that determine the rate of elastic and inelastic scattering within the target and horn. The outgoing final state configurations of these interactions are handled by the default Geant 4 elastic and inelastic scattering algorithms, with the exception of the primary $p$-Be interactions.

For other particle/nucleus combinations, the default cross section tables in Geant4 are used. Extensive checks have been performed to ensure that the rate of hadronic interactions, both elastic and inelastic, are consistent with cross sections assigned to these processes. The final state configurations of neutrinos produced in decays are handled outside of the Geant4 framework as described in Sec. III G.

\section{E. Primary proton interactions}

For the vast majority of primary protons, the first beam line component encountered is the target. Since $p$-Be interactions are the primary source of secondary mesons, a dedicated model (described in Sec. V) tuned to external 
data is used to describe the particle production in proton interactions on the target slugs, fins and sleeve. Elastic scattering is handled by existing Geant 4 models, while nucleon and pion quasielastic scattering use a dedicated model based on free hadron-nucleus scattering data. Because of the divergence of the primary beam and scattering, it is possible for a primary proton to interact outside of the target (usually the aluminum of the horn or the concrete of the decay region). For these cases, the particle production is handled by the default Geant 4 hadronic model.

For the primary $p$-Be interactions, secondary particles of seven types $\left(\pi^{ \pm}, K^{ \pm}, K^{0}, p, n\right)$ are generated according to custom production tables describing the double differential cross sections for the production of each secondary species as a function of $p_{z}$ and $p_{T}$, the components of momentum along and transverse to the primary proton direction, respectively. The total production cross section for a given species, obtained by integrating the double differential cross section, determines the average multiplicity of the species in each primary $p$-Be reaction (hadronic interactions excluding elastic and quasielastic scattering) when divided by the reaction cross section. In each such reaction, the multiplicity for each species in drawn from a Poisson distribution based on the average multiplicity, and the kinematics drawn from the table of double differential cross sections. The reaction cross sections are described in Sec. IV, while the specification of the double differential cross section tables is described in Sec. V.

\section{F. Particle decays}

Neutrinos reaching the MiniBooNE detector are produced in the decays of charged pions, charged and neutral kaons, and muons. The particle lifetimes, decay modes and associated branching ratios, and kinematic distributions of the neutrinos produced in the decays assumed in the simulation affect neutrino flux predictions, and are discussed here. The neutrino parent lifetimes and branching ratios used in the simulation are given in Table $\mathrm{I}$, for $\pi^{+}, K^{+}, K_{L}^{0}$,

TABLE I. Particle lifetimes, and neutrino-producing decay modes and branching ratios considered in the simulation.

\begin{tabular}{lccc}
\hline \hline Particle & Lifetime (ns) & Decay mode & Branching ratio (\%) \\
\hline$\pi^{+}$ & \multirow{2}{*}{26.03} & $\mu^{+}+\nu_{\mu}$ & 99.9877 \\
& & $e^{+}+\nu_{e}$ & 0.0123 \\
$K^{+}$ & \multirow{2}{*}{12.385} & $\mu^{+}+\nu_{\mu}$ & 63.44 \\
& & $\pi^{0}+e^{+}+\nu_{e}$ & 4.98 \\
& & $\pi^{0}+\mu^{+}+\nu_{\mu}$ & 3.32 \\
$K_{L}^{0}$ & \multirow{2}{*}{51.6} & $\pi^{-}+e^{+}+\nu_{e}$ & 20.333 \\
& & $\pi^{+}+e^{-}+\bar{\nu}_{e}$ & 20.197 \\
& & $\pi^{-}+\mu^{+}+\nu_{\mu}$ & 13.551 \\
$\mu^{+}$ & \multirow{2}{*}{2197.03} & $\pi^{+}+\mu^{-}+\bar{\nu}_{\mu}$ & 13.469 \\
\hline \hline
\end{tabular}

and $\mu^{+}$neutrino parents. The corresponding decays of negatively-charged particles are also simulated.

In the two-body decays of charged pseudoscalar mesons $M^{+} \rightarrow l^{+}+\nu_{l}$, where $M=\pi, K$ and $l=e, \mu$, neutrinos are produced in the meson rest frame with fixed energy $E_{\nu}=\left(m_{M}^{2}-m_{l}^{2}\right) /\left(2 m_{M}\right)$ and isotropic angular distribution.

For kaon semileptonic decays, $K \rightarrow \pi+l+\nu_{l}$ (" $K_{l 3}$ "), neutrinos are produced with isotropic angular distribution in the kaon rest frame. For the neutrino energy distributions, different parametrizations are used for the $K_{l 3}^{ \pm}$and $K_{l 3}^{0}$ form factors depending on whether electron or muon neutrinos are produced in the decay. In both cases, we assume that only vector currents contribute, and that time-reversal invariance holds.

For $K_{e 3}^{ \pm}$and $K_{e 3}^{0}$ decays, the electron neutrino energy distribution in the kaon rest frame is given by [15]:

$$
\frac{d N}{d E_{\nu}} \propto \int_{E_{e,-}}^{E_{e,+}} d E_{e}\left(2 E_{e} E_{\nu}-m_{k} E_{\pi}^{\prime}\right)\left|f_{+}^{e}(t)\right|^{2}
$$

where $E_{e}$ is the electron energy, $f_{+}$is a form factor depending on the square of the four-momentum transfer to the pion, $t=\left(p_{K}-p_{\pi}\right)^{2}=m_{K}^{2}+m_{\pi}^{2}-2 m_{K} E_{\pi}$, $E_{\pi}^{\prime}$ is given by:

$$
E_{\pi}^{\prime} \equiv \frac{m_{K}^{2}+m_{\pi}^{2}-m_{e}^{2}}{2 m_{K}}-E_{\pi}
$$

and $E_{e, \pm}$ are integration limits on the electron energy:

$$
E_{e,-}=\frac{m_{K}^{2}-m_{\pi}^{2}}{2 m_{k}}-E_{\nu}, \quad E_{e,+}=\frac{1}{2}\left(m_{k}-\frac{m_{\pi}^{2}}{m_{k}-2 E_{\nu}}\right)
$$

We assume a linear dependence of the form factor $f_{+}^{e}$ on $t$ :

$$
f_{+}^{e}(t)=f_{+}^{e}(0)\left(1+\lambda_{+}^{e} t / m_{\pi}^{2}\right)
$$

For both $K_{e 3}^{+}$and $K_{e 3}^{0}$ decays, the coefficient $\lambda_{+}^{e}$ for the linear expansion of the form factor used is $2.82 \times 10^{-2}$ [16].

For $K_{\mu 3}^{ \pm}$and $K_{\mu 3}^{0}$ decays, the muon neutrino energy distribution in the kaon rest frame is given by [15]:

$$
\frac{d N}{d E_{\nu}} \propto \int_{E_{\mu,-}}^{E_{\mu,+}} d E_{\mu}\left|f_{+}^{\mu}(t)\right|^{2}\left[A+B \xi(t)+C \xi(t)^{2}\right],
$$

where:

$$
\begin{gathered}
A \equiv m_{K}\left(2 E_{\mu} E_{\nu}-m_{K} E_{\pi}^{\prime}\right)+m_{\mu}^{2}\left(E_{\pi}^{\prime} / 4-E_{\nu}\right), \\
B \equiv m_{\mu}^{2}\left(E_{\nu}-E_{\pi}^{\prime} / 2\right), \quad C \equiv m_{\mu}^{2} E_{\pi}^{\prime} / 4 .
\end{gathered}
$$

The quantities $E_{\pi}^{\prime}, E_{\mu,-}, E_{\mu,+}, f_{+}^{\mu}(t)$ appearing in Eqs. (5) and (6) are defined as in the $K_{e 3}$ case (see 
Eqs. (2)-(4)) substituting $e \rightarrow \mu$. In the simulation, we take $\xi(t) \simeq \xi(0)=-0.19[16]$.

Concerning electron (anti-)neutrinos from $\mu^{ \pm}$decays, we neglect terms proportional to the electron mass and assume the following neutrino energy and angular distribution [17]:

$$
\frac{d N}{d x d \Omega_{\nu}}=\frac{12 x^{2}}{4 \pi}(1-x)\left[1 \mp P_{z} \cos \left(\theta_{\nu}\right)\right],
$$

where $\cos \left(\theta_{\nu}\right)$ is the neutrino emission angle with respect to the beam direction $z, P_{z}$ is the projection along $z$ of the muon polarization vector in the muon rest frame, and $x=$ $2 E_{\nu} / m_{\mu}$, with $0<x<1$. The muon polarization vector is estimated on an event-by-event basis. For $\pi^{+} \rightarrow \mu^{+} \rightarrow \nu_{e}$ decays, the muon polarization in the muon rest frame is calculated from the known muon polarization in the pion rest frame, and boosting the polarization vector into the muon rest frame. The muon polarization for muons proceeding from $K^{ \pm}$decays is computed in the same way, with the simplifying assumption that all $K^{ \pm}$decays proceed via the $K_{\mu 2}^{ \pm}$decay mode.

\section{G. Statistical enhancements}

Running the Geant 4 beam line simulation and recording the outgoing neutrinos proton-by-proton would not provide enough neutrinos at the MiniBooNE detector to allow for a precise determination of the flux across the entire phase space of interest. As a result, several modifications are made to enhance the beam Monte Carlo simulation statistics.

A large statistical enhancement is gained by "redecaying" the parent particle of the neutrino. For each neutrino produced in the beam Monte Carlo, the particle decay which produced the neutrino is performed 1000 times. Each redecay is performed at the same location, but the kinematics of the decay are randomly redrawn each time from the decay distributions, resulting in different momenta for the daughter particles in each draw.

A similar technique is used to boost the statistics of neutrinos from muon decay. Most muons produced in the secondary beam do not decay before stopping in the beam stop or the walls of the decay region due to their long lifetime. To better estimate the muon decay-in-flight component of the neutrino flux, each time a muon is produced in the simulation, 19 identical copies are created and independently propagated through the simulation. To account for the resulting overproduction of neutrinos, the weight for each muon-decay neutrino is correspondingly reduced by a factor of 20 .

Another weighting technique is used to determine the high-energy neutrino flux. The MiniBooNE neutrino energy spectrum peaks between 500 and $600 \mathrm{MeV}$ with a long high-energy tail extending past $6 \mathrm{GeV}$. Since fewer neutrinos are produced at these higher energies, statistical fluctuations are much larger, increasing the uncertainty in the shape of the high-energy tail. This problem is made worse by the redecay procedure described above since high-energy parent particles tend to decay to high-energy, forward-going neutrinos, which resulting in a significant fraction of the 1000 redecays producing neutrinos pointed at the detector, all with similar energies. To reduce the statistical uncertainty in the prediction of the high-energy tail, the meson production cross sections for protonberyllium interactions are multiplied by an exponential function in longitudinal meson momentum. Each event is deweighted by its corresponding cross section enhancement to preserve the correct neutrino/proton ratio. This provides an artificially large production of neutrinos at high energies with small event weights, thus reducing the statistical uncertainty in the high-energy tail of the predicted neutrino flux.

The neutrino flux at the MiniBooNE detector is determined by projecting the path of the neutrino to the plane containing the center of the detector, 541 meters from the face of the target. Neutrinos which are on a path to pass through the detector (within $610.6 \mathrm{~cm}$ of the center of the detector at this plane, accounting for the vertical displacement) are recorded in the flux distributions used for Monte Carlo event simulation in the detector. For the simulation of neutrino interactions outside the detector in the concrete walls of the detector hall or in the dirt beyond, a larger radius of $1400 \mathrm{~cm}$ is used to determine the flux distributions.

\section{HADRONIC INTERACTIONS IN THE BEAM LINE}

Hadronic cross sections play an important role in determining the properties of the neutrinos produced in the BNB. Most notably, hadronic cross sections on beryllium and aluminum, the materials composing the target and horn, respectively, govern the rate of primary proton interactions on the target, as well as the rate of absorption of pions in the target and the horn. The breakdown of the proton cross sections between elastic, quasielastic and other forms of interactions govern the fraction of protons that scatter out of the target before interacting. The analogous breakdown of the cross section for pions is particularly important at high momentum, where forward-going pions may intersect a considerable amount of material in the target or the horn before entering the decay region.

The cross sections fall into three categories: elastic (coherent) scattering, inelastic scattering, and quasielastic scattering. In the first, the incident hadron scatters coherently from the nucleus as a whole. The rest of the total hadronic cross section is due to inelastic processes. A subset of these processes involve hadron scattering with the nucleons within the nucleus in a manner analogous to the elastic scattering of hadrons off free nucleons; this is referred to as quasielastic scattering. The remainder of the 
TABLE II. Origin of hadron-beryllium cross sections used in the Geant4 simulation. "Glauber" indicates that the Glauber model calculations described in Sec. IVA are used. These have been cross checked by $n$-Be $\sigma_{\text {TOT }}$ data. "Data" indicates that existing measurements are directly parametrized. "Shadow" refers to the calculation of $\sigma_{\mathrm{QEL}}$ using the shadowed multiple scattering model described in Sec. IV B.

\begin{tabular}{lccc}
\hline \hline & $p$-(Be/Al) & $n$-(Be/Al) & $\pi^{ \pm}-(\mathrm{Be} / \mathrm{Al})$ \\
\hline$\sigma_{\text {TOT }}$ & Glauber & Glauber & Data $(p<0.6 / 0.8 \mathrm{GeV} / c)$ \\
& Data & Glauber $(p>0.6 / 0.8 \mathrm{GeV} / c)$ \\
$\sigma_{\text {INE }}$ & Shadow & (same as $p$-Be/Al) & Data \\
$\sigma_{\mathrm{QEL}}$ & Shadow & Data $(p<0.5 \mathrm{GeV} / c)$ \\
& & Shadow $(p>0.5 \mathrm{GeV} / c)$ \\
\hline \hline
\end{tabular}

inelastic cross section includes the particle production processes discussed in Sec. V. The relevant momentum range in the flux prediction are at and below the primary proton momentum $(8.89 \mathrm{GeV} / c)$ for nucleons. The corresponding momentum range for the pions produced by these protons is $0-6 \mathrm{GeV} / c$

Wherever possible, measured cross sections have been used in the simulation. In some cases, the measured and calculated cross sections are extrapolated to cross sections for other particles that are related by isospin. Measurements exist primarily for total hadronic cross sections and inelastic cross sections, from which the elastic cross section can be inferred. Theoretical guidance is needed primarily for the total hadronic cross sections for pionnucleus scattering and quasielastic scattering. Table II summarizes the source of nucleon and pion cross sections. Details of the parametrizations used to describe the momentum dependence of each cross section are given in Sec. IV C.

\section{A. Total and elastic scattering}

The elastic scattering cross sections for protons, neutrons and charged pions have been obtained by calculating the total hadronic cross section $\sigma_{\text {TOT }}$ using the Glauber model [18] and subtracting the measured inelastic cross sections described in Sec. IV B assuming $\sigma_{\mathrm{TOT}}=\sigma_{\mathrm{INE}}+$ $\sigma_{\mathrm{ELA}}$. Direct measurements of $\sigma_{\mathrm{TOT}}$ for hadron-nucleus interactions in the relevant energy range exist only for neutrons and for pions in the $\Delta(1232)$ resonance region. Wherever possible, we compare the calculated results with the existing measurements to check their validity. No direct measurements of the total elastic cross section $\left(\sigma_{\mathrm{ELA}}\right)$ exist in this momentum range.

The calculation of $\sigma_{\text {TOT }}$ follows the work described in Ref. [19], where hadron-nucleus elastic scattering is modeled as the coherent sum of scattering amplitudes from hadron-nucleon scattering. The amplitude for forward elastic scattering is calculated allowing $\sigma_{\mathrm{TOT}}$ to be obtained via the optical theorem. At each incident hadron energy, these amplitudes are summarized by three parameters, namely, the total cross section for hadron-nucleon scattering $\left(\sigma_{n}\right)$, the ratio of the real and imaginary parts of the forward scattering amplitude $(\alpha)$, and the differential cross section in $t=\left|q^{2}\right|$, the square of the four-momentum transfer. The latter is parametrized as an exponential distribution in $t$. All together, the hadron-nucleon scattering amplitude can be expressed as:

$$
f(q)=\frac{(i+\alpha) k \sigma_{n}}{4 \pi} e^{\beta t / 2},
$$

where $k$ is the wave number of the incident hadron. This form identically satisfies the optical theorem.

The Glauber model for elastic scattering represents the nucleus as a collection of nucleons distributed in a spherically symmetric state with radial distributions given by the independent harmonic oscillator form (for beryllium) or the Woods-Saxon form (for aluminum) [20]. The scattering amplitude for a given configuration of nucleons is obtained by considering the phase shift due to the individual hadronnucleon scattering amplitudes. The total scattering amplitude for the nucleus is calculated by averaging over all nucleon configurations weighted by the nucleon density distribution. The total cross section $\sigma_{\mathrm{TOT}}$ is obtained by applying the optical theorem to the resulting forward scattering amplitude. As mentioned above, $\sigma_{\text {ELA }}$ at a particular incident hadron momentum is calculated via the relation $\sigma_{\mathrm{TOT}}=\sigma_{\mathrm{ELA}}+\sigma_{\mathrm{INE}}$ using the values of $\sigma_{\mathrm{INE}}$ described below. While it is in principle possible to obtain $\sigma_{\mathrm{ELA}}$ from the Glauber model by obtaining the elastic cross section as a function of $q^{2}$ and integrating, the assumptions of the model are most valid in the forward direction. An extraction of $\sigma_{\mathrm{ELA}}$ using the model requires integrating the differential cross section outside of this region.

The hadron-nucleon scattering parameters $\sigma_{n}, \alpha$ and $\beta$ are obtained from the literature. In particular, $\sigma_{n}$ and $\alpha$ for $p-p, p-n, \pi^{+}-p$ and $\pi^{-}-p$ elastic scattering have been compiled by the Particle Data Group (PDG) [21]. The compiled data on $\sigma_{n}$ and $\alpha$ and our parametrization of their momentum dependences are shown in Figs. 7 and 8. In addition to the PDG compilation, a compilation of $\alpha$ measurements by CERN [22], as well as the measurements of Foley et al. [23], have been included in the parametri- 

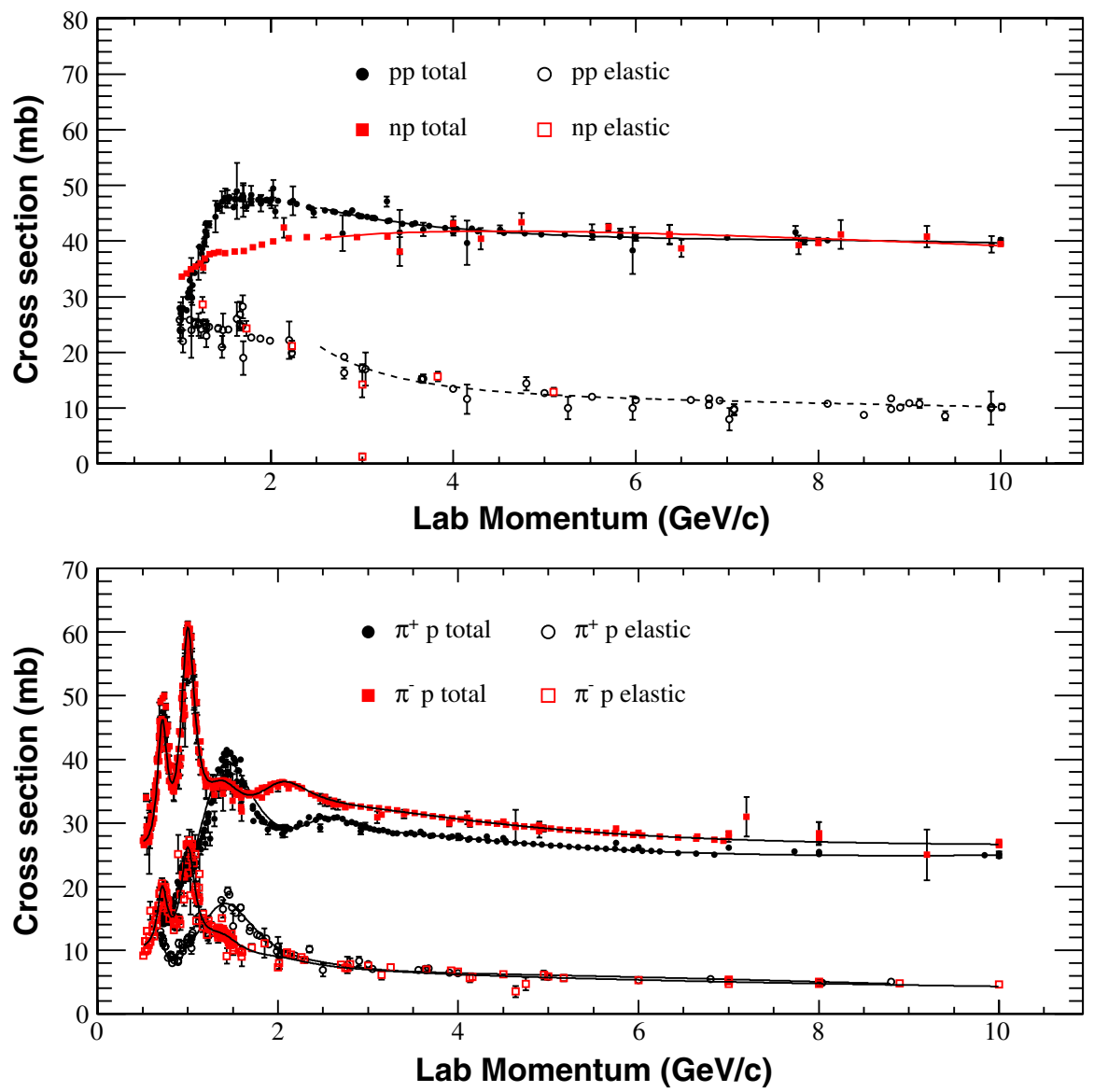

FIG. 7 (color online). Total and elastic hadron-proton cross sections (Top: proton/neutron, Bottom: $\pi^{+} / \pi^{-}$) compiled by the Particle Data Group and the COMPAS collaboration [21], with the parametrizations used in the Glauber model calculations.

zation. This latter data are at momenta above the region of interest $([7-10] \mathrm{GeV} / c)$ but are nonetheless useful in determining the momentum evolution of $\alpha$ for $\pi^{ \pm}-p$ scattering. The measurements at momenta less than $3.5 \mathrm{GeV} / c$ come entirely from the CERN compilation. The PDG compilation of $\alpha$ in nucleon-nucleon scattering adequately covers the range of interest for the flux prediction.

Unfortunately, the $\beta$ measurements for hadron-nucleon elastic scattering have not been compiled by the PDG. We have taken data from a number of experiments (for $p-p$ [24-27], for $n-p[28,29]$, and for $\left.\pi^{ \pm}-p[22,24,30]\right)$

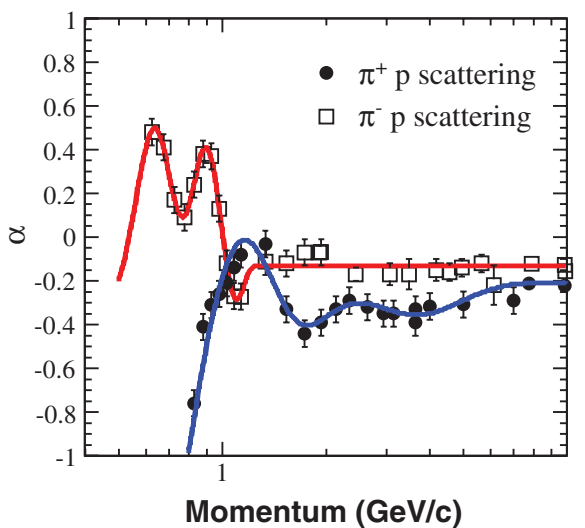

FIG. 8 (color). Measured values of $\alpha$ the real-to-imaginary ratio of the forward scattering amplitude for $p-p$ and $n-p$ scattering (left) and $\pi^{+}-p$ and $\pi^{-}-p$ scattering (right) with parametrizations. The parametrizations used in the Glauber model calculation are shown. 

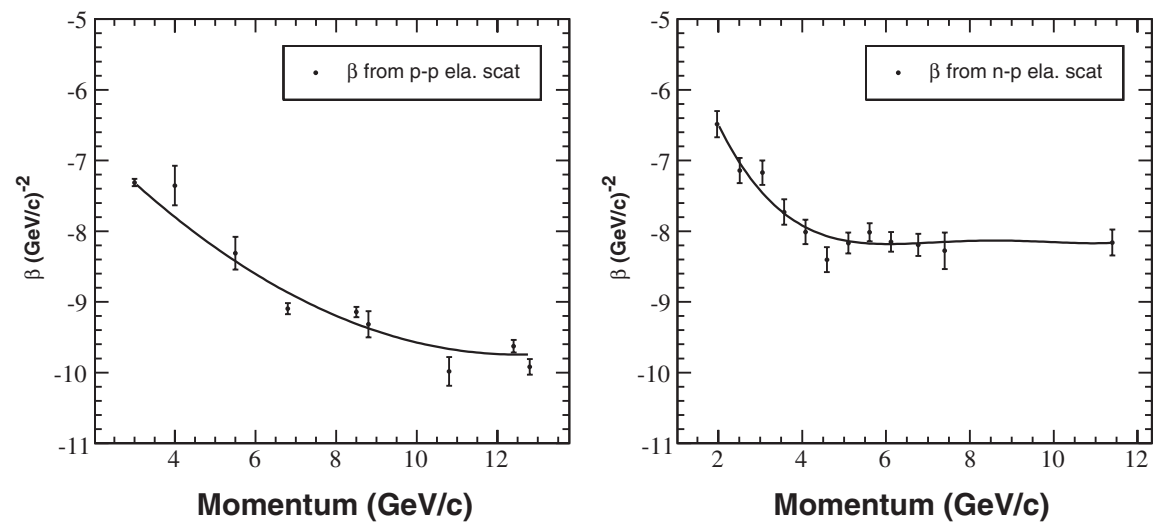

FIG. 9. $\beta$ parameters for $p-p$ (left) and $n-p$ (right) scattering obtained from fits to the data with the parametrizations used in the Glauber model calculation.
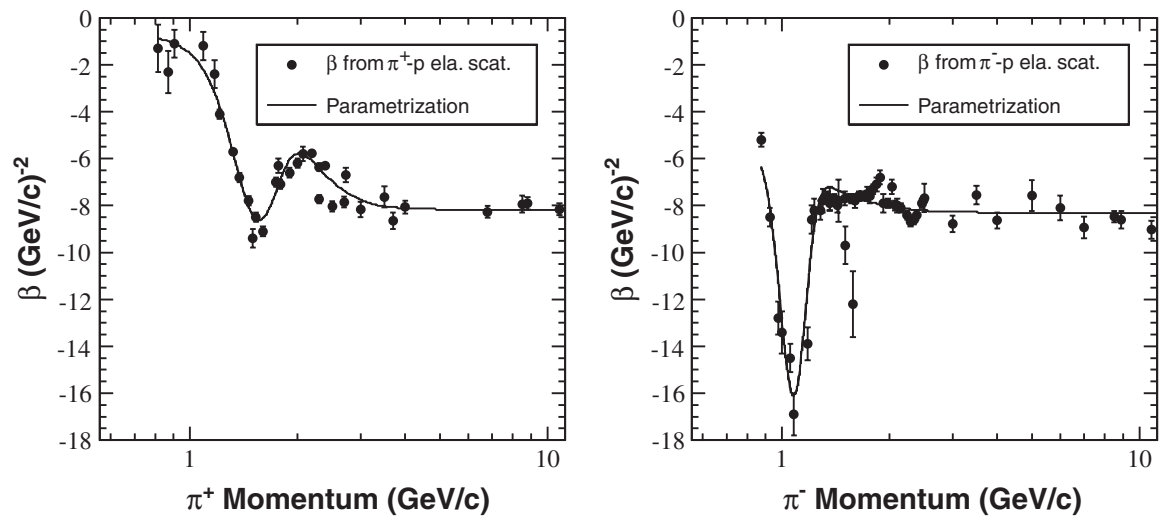

FIG. 10. Compilation of measured $\beta$ parameters for $\pi^{+}-p$ elastic scattering (left) and $\pi^{-}-p$ elastic scattering (right) versus incident pion momentum with the parametrizations used in the Glauber model calculation. The measured values of $\beta$ include the Lasinski compilation [31] as well as our own fits to the $q^{2}$ distributions measured in Refs. [22,24,30].
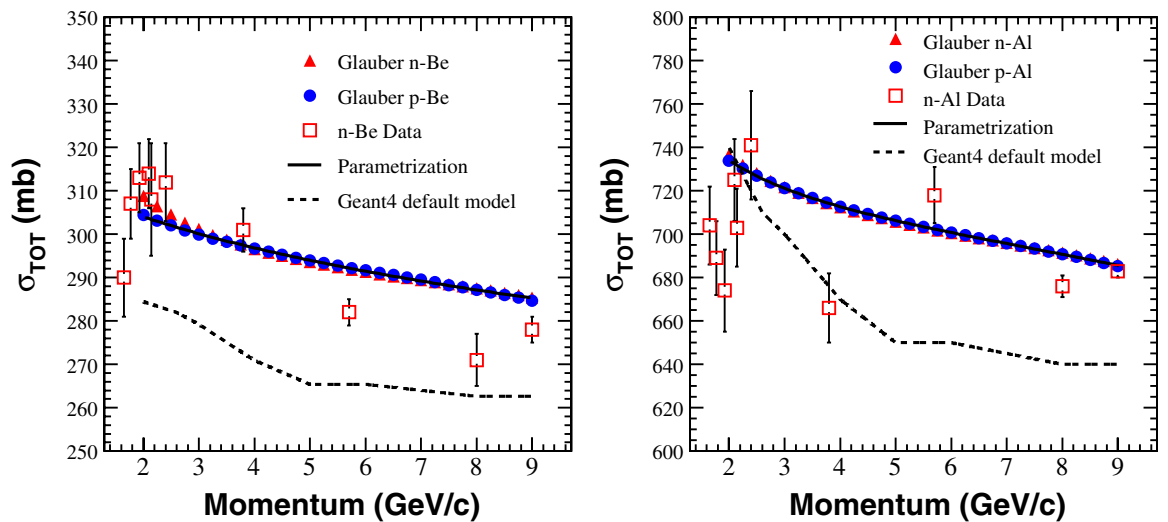

FIG. 11 (color online). Total hadronic cross sections calculated using the Glauber model and the optical theorem for beryllium (left) and aluminum (right) targets. The calculated results for neutrons (protons) are shown as triangles (circles). The parametrization used in the flux prediction is shown as the solid line, while the default Geant4 parametrization is shown as a dashed line. The measured values of $\sigma_{\mathrm{TOT}}$ for $n$-Be/Al from Refs. [62-66] are shown as squares.

and used the reported $t$ distributions to extract $\beta$. Further, a compilation by Lasinski et al. [31] is used to supplement our own compilation for $\pi^{ \pm}-p$ scattering at low momentum. The compiled $\beta$ values and the parametrized momen- tum dependences are shown in Fig. 9 for $p-p$ and $n-p$ scattering, and Fig. 10 for $\pi^{ \pm}-p$ scattering.

The resulting total cross sections for nucleon-nucleus scattering (beryllium and aluminum) are shown in Fig. 11. 

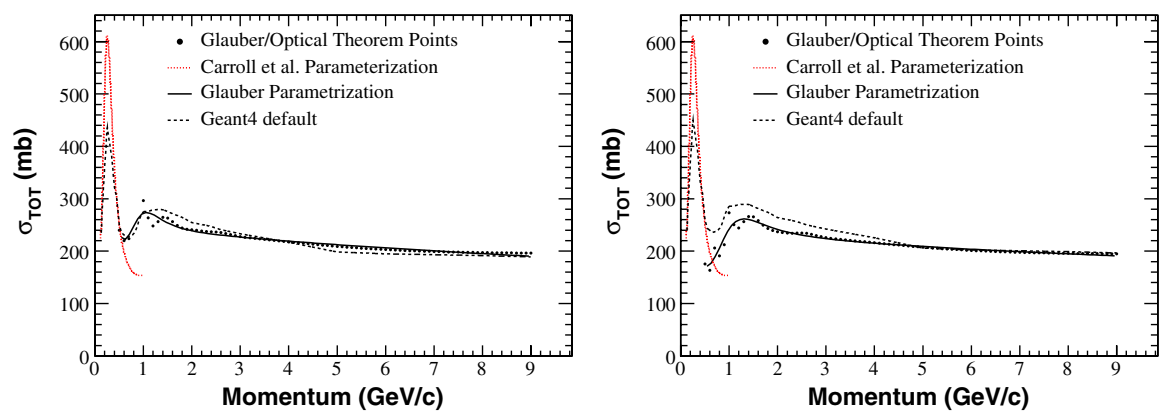

FIG. 12 (color online). Total hadronic cross sections for $\pi^{ \pm}$-Be calculated using the Glauber model (points) for $\pi^{+}$(left) and $\pi^{-}$ (right). The Breit-Wigner parametrization based on the Carroll data [32] on the $\Delta(1232)$ resonance is shown as a dotted line, while the parametrization of the Glauber model points is shown as a solid line. The Geant 4 default model is shown as a dashed line.
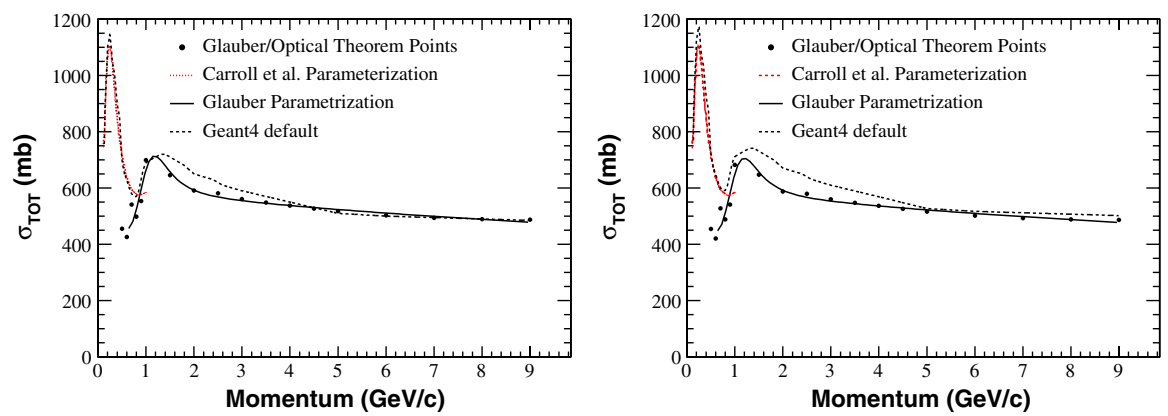

FIG. 13 (color online). Total hadronic cross sections for $\pi^{ \pm}-\mathrm{Al}$ calculated using the Glauber model (points) for $\pi^{+}$(left) and $\pi^{-}$ (right). The Breit-Wigner parametrization based on the Carroll data [32] on the $\Delta(1232)$ resonance is shown as a dotted line, while the parametrization of the Glauber model points is shown as a solid line. The Geant 4 default model is shown as a dashed line.

The calculated values of $\sigma_{\text {TOT }}$ are compared with measurements of $\sigma_{\text {TOT }}$ for $n$-Be data. The model predictions agree with the data to within several percent, and indicate that $\sigma_{\text {TOT }}$ for proton-nucleus and neutron-nucleus interactions are very similar except at the lowest energies, as expected from isospin symmetry. The success of the model in reproducing $\sigma_{\text {TOT }}$ neutron-nucleus is taken as an indication that the model can be used for proton-nucleus and pion-nucleus interactions, where such a check with data is not possible. The spread in values between the data and the model is considered a source of systematic uncertainty.

The $\sigma_{\text {Tот }}$ values obtained for pion-nucleus interactions are shown in Fig. 12 for $\pi^{ \pm}$-Be interactions and Fig. 13 for $\pi^{ \pm}-\mathrm{Al}$ interactions. The calculated points are parametrized by the black curve. At low momentum $(<600 \mathrm{MeV} / c$ for beryllium, $<800 \mathrm{MeV} / c$ for aluminum), where the $\Delta$ resonance dominates the cross section, parametrizations based on $\sigma_{\text {TOT }}$ measurements by Carroll et al. are used [32]. While not used in the flux prediction, the $\sigma_{\text {TOт }}$ values used in the GHEISHA model (the Geant4 default) are shown as a dashed black line for comparison.

\section{B. Inelastic and quasielastic processes}

In the case of inelastic scattering $\left(\sigma_{\text {INE }}\right)$, a much larger set of cross section measurements exists eliminating the need for theoretical models. The entire momentum range of interest for nucleon-nucleus inelastic scattering and a large subset of the momentum range for pion-nucleus inelastic scattering has been measured.

The available measurements of $\sigma_{\mathrm{INE}}$ for $p$-Be and $p$-Al interactions in the relevant momentum range are shown in Fig. 14. The Gachurin et al. data [33] spans the low momentum region, while the Bobchenko et al.[34] data covers the high momentum region up to $9 \mathrm{GeV} / c$. Together, they cover the entire momentum range of interest for MiniBooNE. The parametrization used to model the momentum dependence is shown as a solid black line. Likewise, $\sigma_{\text {INE }}$ for $\pi^{ \pm}$-nucleus interactions are shown in Fig. 15. The Ashery et al. [35] measurements are used around the $\Delta$ resonance, while the Allardyce et al. [36], Gachurin et al. [33], and Bobchenko et al. [34] data are used at higher momentum. The low momentum data do not include beryllium; for these points, the cross sections are extrapolated using the cross sections measured on different elements at the same momentum. The measured cross sections are parametrized as $A^{n}$, where $n$ has been determined from the $A$-dependence of the measured cross section at each momentum from Ref. [35]. Typical values of $n$ range from 0.6 to 0.8 . The resulting function is used to infer the cross section at $A=9$.

A subset of the inelastic interactions results from quasielastic scattering, where hadrons scatter off the individual 

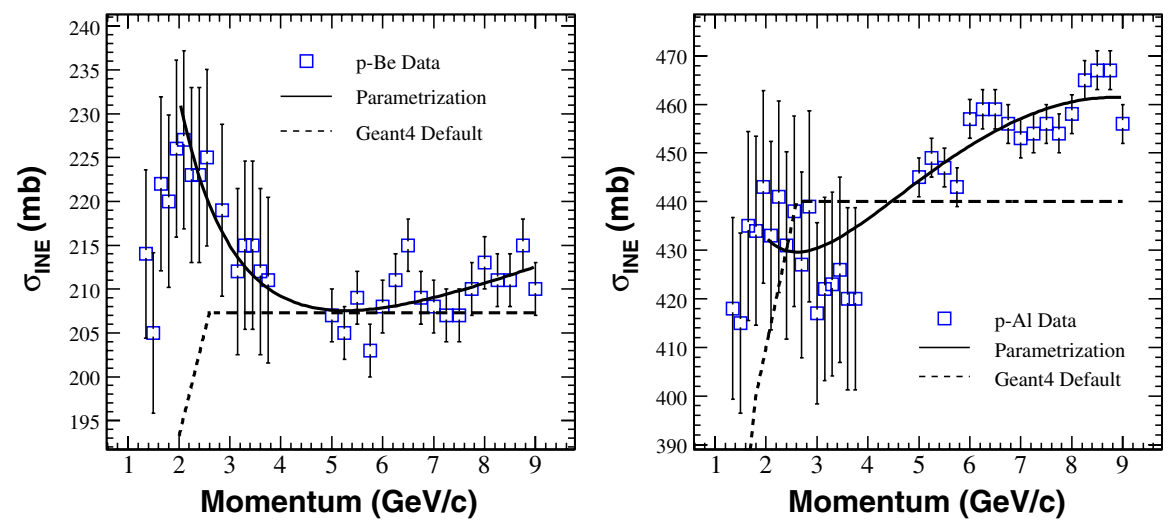

FIG. 14 (color online). $\quad$-Be (left) and p-Al (right) inelastic cross sections measured from Gachurin et al. [33] (1-4 GeV/c) and Bobchenko et al. [34] (5-9 GeV/c). The solid line is the parametrization used in the flux prediction, while the dashed line shows the Geant4 default parametrization.

nucleons in the nucleus in a manner analogous to hadron elastic scattering off free nucleons. The rate of this process relative to other forms of inelastic scattering is important since it allows the incoming hadron to emerge from inelastic scattering with its initial momentum largely intact, whereas it would otherwise be effectively absorbed or significantly reduced in momentum.
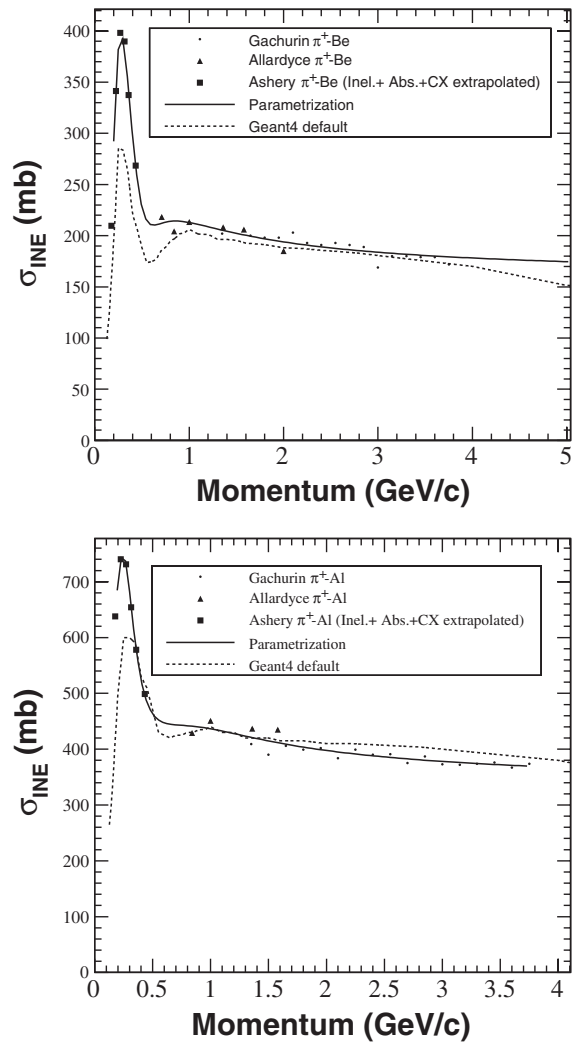

Unfortunately, the available measurements of $\sigma_{\mathrm{QEL}}$ are sparse, with only a few measurements for pions at low momentum. As a result, we must appeal to a theoretical calculation for this part of the inelastic cross section. This can be effected via the shadowed multiple scattering expansion, in which $\sigma_{\mathrm{QEL}}$ is calculated as the incoherent sum of the cross section for hadrons to scatter off the nucleons in the nucleus, accounting for the attenuation of the hadron
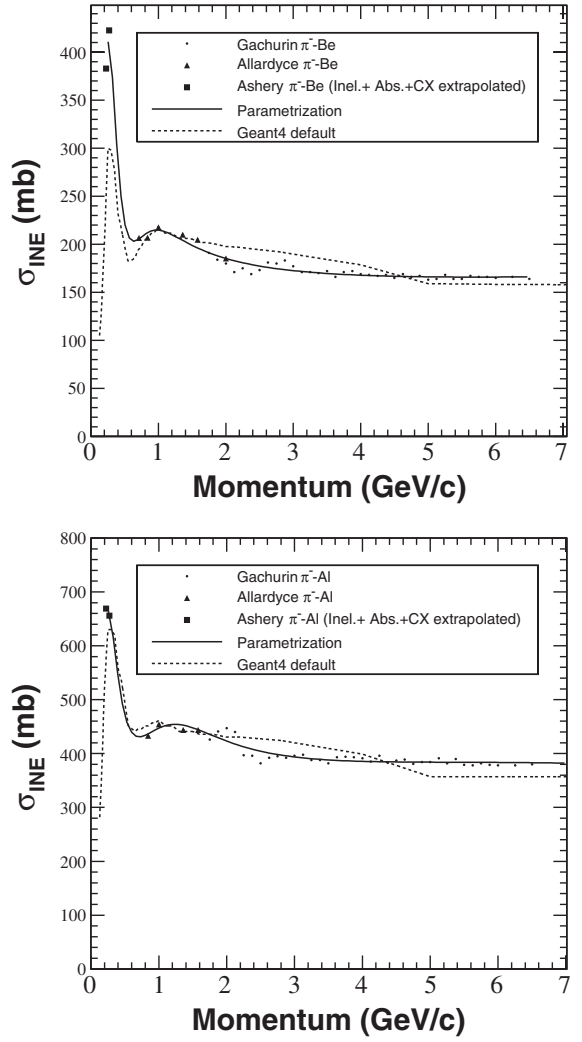

FIG. 15. Inelastic cross sections for $\pi^{+}$-Be (top left), $\pi^{-}$-Be (top right), $\pi^{+}$-Al (bottom left) and $\pi^{-}$-Al (bottom right) as measured in Refs. [35] (squares), [36] (triangles), and [34] (circles). The solid lines are the parametrizations used in the flux prediction, while the dashed lines are the default Geant 4 parametrizations. 

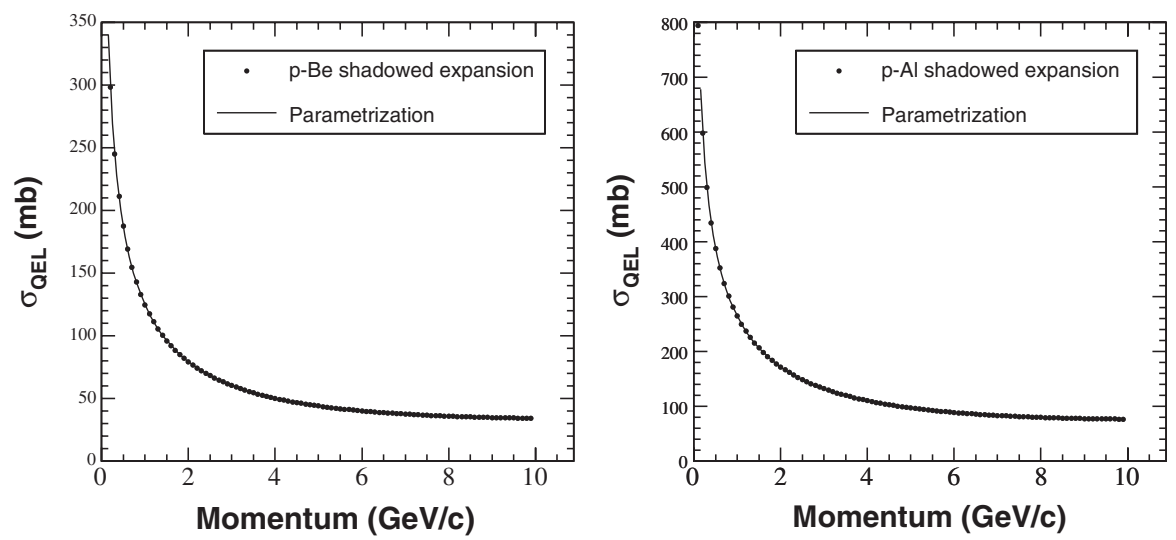

FIG. 16. Calculated values of $\sigma_{\mathrm{QEL}}$ for $p$-Be (left) and $p$-Al (right) interactions along with the parametrization used in the flux prediction.

wave function as it traverses the nucleus [18]. The cross section for multiple scattering of the hadron within the same nucleus can also be calculated in this formalism. This is found to be a small fraction of the single-scatter cross section in our case.

The calculated values of $\sigma_{\mathrm{QEL}}$ for nucleon-nucleus quasielastic scattering are shown in Fig. 16, while the values for pion-nucleus scattering from Ref. [35] are shown in
Fig. 17. The latter figure includes measurements of $\sigma_{\mathrm{QEL}}$ for $\pi^{ \pm}$-nucleus interactions around the $\Delta$ resonance. The calculated values, along with the measurements, have been incorporated into the parametrizations of the momentum dependence of $\sigma_{\mathrm{QEL}}$ for each of the hadron/nucleus combinations. As before, these measurements do not exist for beryllium and have been extrapolated assuming an $A^{n}$ dependence, where $n$ has been determined from the
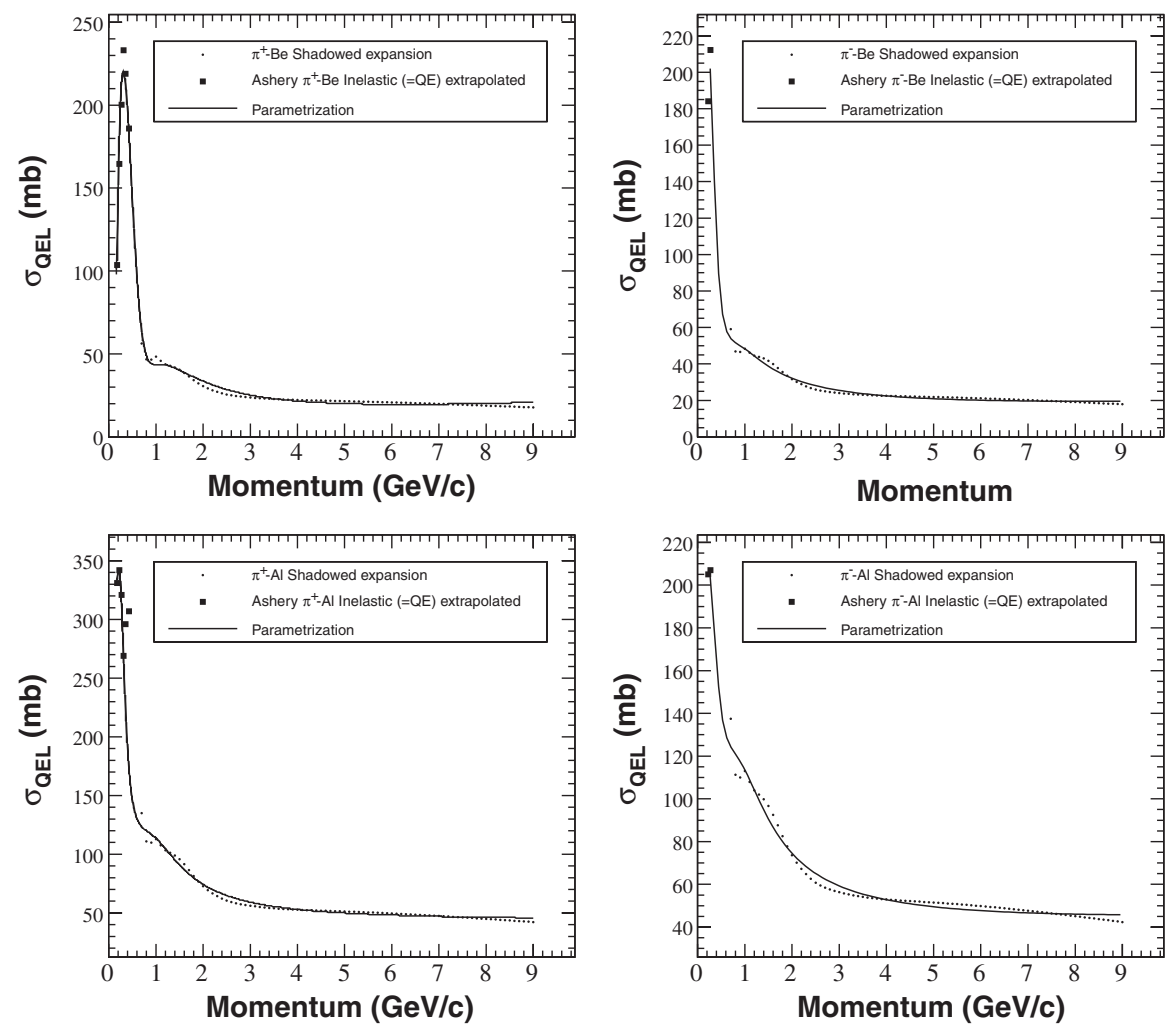

FIG. 17. Quasielastic cross sections for $\pi^{+}$-Be (top left), $\pi^{-}$-Be (top right), $\pi^{+}$-Al (bottom left) and $\pi^{-}$-Al (bottom right) as measured in Refs. [35] (squares) and calculated using the shadowed scattering model (circles). The solid black lines are the parametrizations used in the flux prediction. 
TABLE III. Parameter values for proton and neutron on beryllium and aluminum cross sections using Eq. (9).

\begin{tabular}{lccccc}
\hline \hline & $A$ & $B$ & $n$ & \multicolumn{1}{c}{$C$} & $D$ \\
\hline$(p / n)-\mathrm{Be}$ & & & & & \\
$\sigma_{\mathrm{TOT}}$ & 307.8 & 0.897 & 0.003 & -2.598 & -4.973 \\
$\sigma_{\mathrm{INE}}$ & 186.7 & 104.3 & -1.039 & 10.38 & -15.83 \\
$\sigma_{\mathrm{QEL}}$ & 164.8 & -40.09 & 0.408 & 21.40 & -61.45 \\
$(p / n)-\mathrm{Al}$ & & & & & \\
$\sigma_{\mathrm{TOT}}$ & 760.3 & -0.056 & 2.485 & 6.173 & -41.60 \\
$\sigma_{\mathrm{INE}}$ & 470.9 & -0.259 & 2.429 & 48.86 & -87.19 \\
$\sigma_{\mathrm{QEL}}$ & 255.7 & 8.792 & 0.0024 & 32.24 & -155.9 \\
\hline \hline
\end{tabular}

A-dependence of the measured cross section at each momentum from Ref. [35]. The resulting values of $n$ range from 0.3 to 1.0 , varying with incident pion momentum.

\section{Explicit forms for the cross section parametrizations:}

In summarizing the momentum dependence of the nucleon and pion cross sections, we have made use of the following form:

$$
\sigma=A+B \times p^{n}+C \times \ln ^{2} p+D \times \ln p,
$$

where $p$ is the momentum of the incident particle in $\mathrm{GeV} / c$. While this form is inspired by Regge theory [37], it is used as a purely empirical description of the cross section. No physical significance is attributed to the parameters apart from the ability of the parametrization to describe the measured or calculated cross sections with the appropriate parameters. The parameters used in the flux prediction for proton and neutron hadronic cross sections on beryllium and aluminum are given in Table III.

For $\sigma_{\mathrm{INE}}$ and $\sigma_{\mathrm{QEL}}$ in pion beryllium/aluminum scattering, a more complicated form is needed in order to describe the peak in the cross section near the $\Delta$ resonances:

$$
\begin{aligned}
\sigma= & N_{R}\left|\frac{-m(p) \Gamma_{R}}{M_{R}^{2}-m(p)^{2}+i m(p) \Gamma_{R}}\right|^{2} \\
& +\left[1+\tanh \left(\theta_{s}\left(p-\theta_{0}\right)\right)\right] \times\left(A+B p^{n}+C \ln ^{2} p\right)
\end{aligned}
$$

where $p$ is the momentum of the incident particle in $\mathrm{GeV} / c$. The first term describes a Breit-Wigner resonance, where $m(p)$ is the invariant mass of the pion/target nucleon system in $\mathrm{GeV} / c^{2}$ assuming that the target is a nucleon at rest. The second term is a simplified version of Eq. (9) with a threshold behavior described by a hyperbolic tangent function. The threshold function allows the second term to dominate at pion momenta above the $\Delta(1232)$ resonance. Here also, the approach is purely empirical; the parameters, including the resonance terms are extracted in such a way to reproduce as closely as possible the measurements, without assigning any physical significance to any of the parameters. In particular, the various $\Delta$ resonances are not modeled individually. The parameters used in the flux simulation using Eq. (10) are given in Table IV.

TABLE IV. Parameter values for $\pi^{ \pm}$-(Be/Al) hadronic cross sections. For $\sigma_{\text {INE }}$ and $\sigma_{\mathrm{QEL}}$, Eq.

\begin{tabular}{|c|c|c|c|c|c|c|c|c|c|}
\hline & $\theta_{0}$ & $\theta_{s}$ & A & $B$ & $n$ & $C$ & $N_{R}$ & $M_{R}$ & $\Gamma_{R}$ \\
\hline \multicolumn{10}{|l|}{$\pi^{+}-\mathrm{Be}$} \\
\hline$\sigma_{\mathrm{TOT}}$ & 0.814 & 3.418 & 237.6 & 111.3 & -4.186 & -9.792 & - & - & - \\
\hline$\sigma_{\mathrm{INE}}$ & 0.400 & 5.142 & 162.3 & -99.79 & -2.407 & -0.423 & 850.3 & 1.201 & 0.375 \\
\hline $\begin{array}{l}\sigma_{\mathrm{QEL}} \\
\pi^{+}-\mathrm{Al}\end{array}$ & 0.635 & 3.784 & -2.38 & -81.84 & -2.702 & 3.173 & 379.9 & 1.201 & 0.558 \\
\hline$\sigma_{\mathrm{TOT}}$ & 0.931 & 3.186 & 569.1 & 511.3 & -3.79 & -18.50 & - & - & - \\
\hline$\sigma_{\mathrm{INE}}$ & 0.295 & 2.307 & 1537.4 & -1109.4 & 0.057 & 14.40 & 510.7 & 1.189 & 0.185 \\
\hline$\sigma_{\mathrm{QEL}}$ & 0.698 & 2.134 & 40.38 & 89.20 & -1.575 & 0.335 & 229.4 & 1.189 & 0.187 \\
\hline & $\theta_{0}$ & $\theta_{s}$ & $A$ & $B$ & $n$ & $C$ & $N_{R}$ & $M_{R}$ & $\Gamma_{R}$ \\
\hline \multicolumn{10}{|l|}{$\pi^{-}-\mathrm{Be}$} \\
\hline$\sigma_{\mathrm{TOT}}$ & 0.814 & 3.418 & 237.6 & 111.3 & -4.186 & -9.792 & - & - & - \\
\hline$\sigma_{\mathrm{INE}}$ & 0.600 & 2.874 & 92.66 & 112.2 & -0.486 & 7.500 & 371.5 & 1.201 & 0.233 \\
\hline $\begin{array}{l}\sigma_{\mathrm{QEL}} \\
\pi^{-}-\mathrm{Al}\end{array}$ & 0.626 & 2.504 & -1.559 & 46.41 & -0.633 & 1.874 & 189.0 & 1.201 & 0.185 \\
\hline$\sigma_{\mathrm{TOT}}$ & 0.931 & 3.186 & 569.1 & 511.3 & -3.79 & -18.50 & - & - & - \\
\hline$\sigma_{\mathrm{INE}}$ & 0.706 & 1.685 & 997.8 & -457.8 & 0.611 & 233.4 & 446.8 & 1.189 & 0.305 \\
\hline$\sigma_{\mathrm{QEL}}$ & 0.633 & 2.199 & 32.52 & 85.15 & -1.225 & 1.383 & 129.1 & 1.189 & 0.305 \\
\hline
\end{tabular}
(10) is used. For $\sigma_{\mathrm{TOT}}$, the parametrization of Carroll et al. [32] is used at low momentum, while Eq. (9) with a threshold term is used at high momentum. 
As mentioned in Sec. IVA, the parametrization of Carroll et al. [32] is used for the total cross sections of $\pi^{ \pm}$scattering on beryllium and aluminum for momenta up to $600 \mathrm{MeV} / c$ in the former case and $800 \mathrm{MeV} / c$ in the latter. At higher momentum, the second term of Eq. (10) is used with the parameters shown in Table IV.

\section{SECONDARY PARTICLE PRODUCTION CROSS SECTIONS}

The primary source of the neutrino flux at MiniBooNE is the decay of secondary particles produced in $p$-Be interactions. The knowledge of the neutrino flux thus critically depends on the understanding of the meson production in $p$-Be interactions. Most of the $\nu_{\mu}$ flux at the MiniBooNE detector comes from $\pi^{+} \rightarrow \mu^{+}+\nu_{\mu}$ decays, while the $\nu_{e}$ flux is dominated by three-body decays of kaons $\left(K^{+}\right.$and $K_{L}^{0}$ ) and the decay of muons (primarily produced in the decay of pions). The tables in the Monte Carlo simulation describing the double differential cross sections which specify the multiplicity and kinematic properties of the protons, neutrons, $\pi^{ \pm}, K^{ \pm}$and $K_{L}^{0}$ produced in $p$-Be interactions at $8.89 \mathrm{GeV} / c$ are based on hadron production measurements with similar kinematic configurations wherever possible. In the case of $\pi^{ \pm}, K^{+}$and $K^{0}$ production, the double differential cross sections are summarized as parametrizations. The parametrizations are evaluated at each point within the table to determine the corresponding cross sections. For protons, neutrons, and $K^{-}$, the cross sections are based on a model of hadronic interactions.

\section{A. Pion production measurements}

The cross section tables for $\pi^{ \pm}$production in $p$-Be interactions are based on parametrizations of measurements taken by the HARP [38] and BNL E910 [39] experiments. The HARP experiment measured the $\pi^{ \pm}$ differential production cross section for $p$-Be interactions using replicas of the MiniBooNE beryllium target at the same incident proton momentum of $8.89 \mathrm{GeV} / c$. However, since the analysis of the data from the replica targets is not complete, the data used in modeling the pion production is from the thin target run, where a 5\% interaction length beryllium target was measured. The pion tracks are binned in total pion momentum $p_{\pi}$ ranging from 0.75 to $6.5 \mathrm{GeV} / c$ and angle $\theta_{\pi}$ with respect to the incident proton direction from 30 to $210 \mathrm{mrad}$. The measurements from the experiment represent the average differential cross section over the bin. A complete covariance matrix is also reported to account for bin-to-bin correlations in the uncertainties. The quoted normalization uncertainty is $\sigma_{\text {HARP }}=2 \%$.

The left plot of Fig. 18 shows the kinematic distribution (in terms of $\theta_{\pi}$ and $p_{\pi}$ ) from the Monte Carlo simulation of pions produced in the target that decay to produce neutrinos in the MiniBooNE detector. The black square indicates the kinematic range covered by the HARP measurements.

The BNL E910 experiment measured the $\pi^{ \pm}$differential cross section for $p$-Be interactions at three different energies of the incident protons $(6.4,12.3,17.5$ and $17.6 \mathrm{GeV} / c)$. Data is binned in pion momentum $p_{\pi}$ ranging from 0.4 to $5.6 \mathrm{GeV} / c$ and angle $\theta_{\pi}$ from 18 to $400 \mathrm{mrad}$. The extended coverage of the E910 measurements in the forward angular region provides further constraints of the pion production in this kinematic region. A covariance matrix was not reported for these measurements, hence we use a diagonal bin-to-bin covariance matrix. The quoted normalization error is $\sigma_{\mathrm{E} 910}=5 \%$.

The $\pi^{+}$production cross sections (momentum distribution of pion production in bins of production angle) from the two experiments are shown in Figs. 19 (HARP $8.89 \mathrm{GeV} / c), 20(\mathrm{E} 9106.4 \mathrm{GeV} / c)$, and 21 (E910 $12.3 \mathrm{GeV} / c$ ). The corresponding $\pi^{-}$production measurements are shown in Figs. 22-24. While a significant body

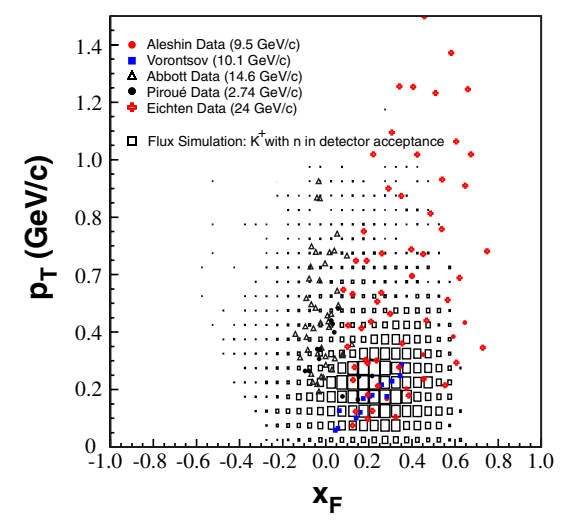

FIG. 18 (color online). Left: Production angle $\theta$ vs momentum $p$ for the $\pi^{+}$in the flux simulation that contribute to the $\nu_{\mu}$ flux at the MiniBooNE detector. The color scale indicates the relative cross section for $\pi^{+}$production in each bin of angle and momentum. The black box marks the kinematic range covered by the HARP measurements [38]. Right: Transverse momentum $p_{T}$ vs the Feynmanscaling variable $x_{F}$ for the $K^{+}$in the flux simulation that contribute to the neutrino flux at the MiniBooNE detector (black squares). The colored points indicate the kinematic regions measured by $p$-Be $K^{+}$production measurements [4]. 


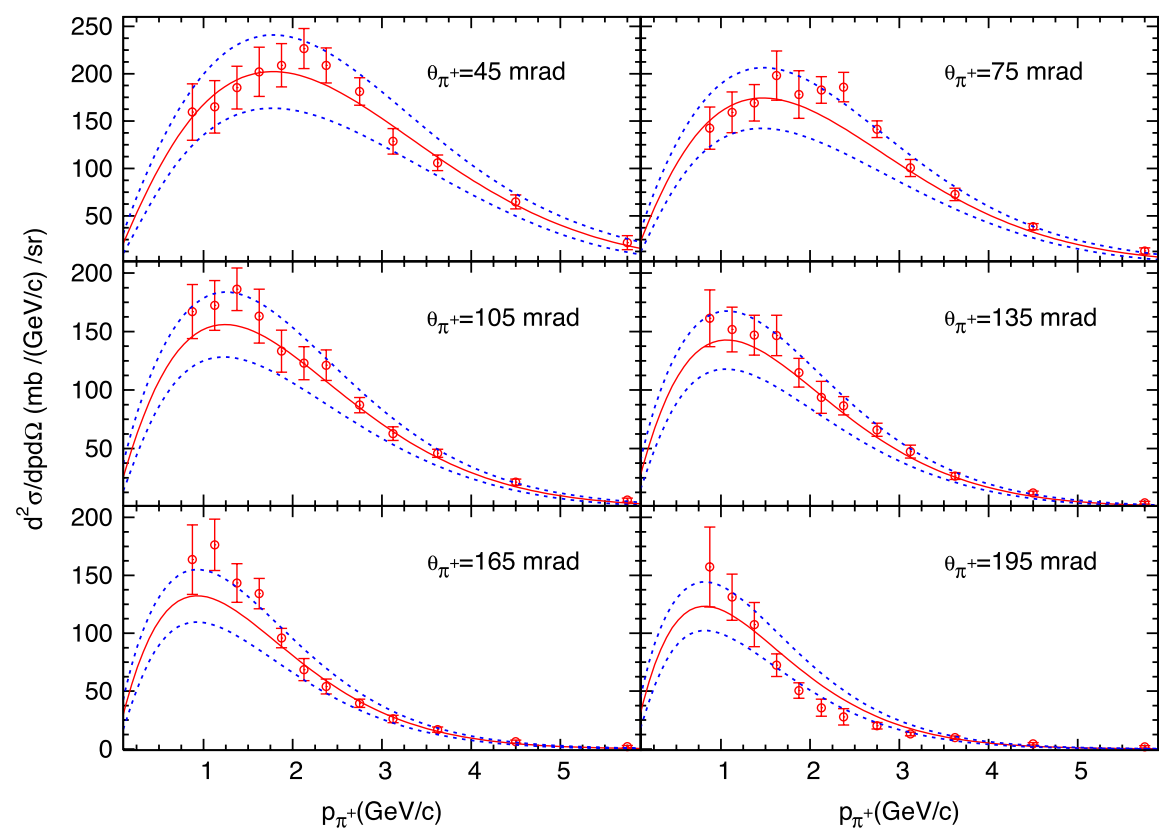

FIG. 19 (color online). Comparison of HARP $\pi^{+}$production cross section data [38] (circles) versus $p_{\pi}$ in bins of $\theta_{\pi}$ from $8.89 \mathrm{GeV} / c p$-Be interactions and best-fit $\mathrm{SW}$ model (solid lines). The dashed lines represent the uncertainty band resulting from varying the parameters within their correlated uncertainties, as described in the text.

of historical $p$-Be pion production data exists ([40-44]), the measurements are removed from the primary beam momentum in the BNB, have insufficient kinematic coverage, or have inconsistencies that led to the exclusive use of the latest data from E910 and HARP. The E910 $17.5 \mathrm{GeV} / c$ and $17.6 \mathrm{GeV} / c$ data is also not used for the first reason.

\section{B. Sanford-Wang fit to the pion production data}

Following the K2K experiment [45], the parametrization of Sanford and Wang (SW) [46] is used to describe the $\pi^{ \pm}$ differential production cross section across different incident primary beam momenta. The SW parametrization for the production cross section of a given meson species is given by

$$
\begin{aligned}
\frac{d^{2} \sigma}{d p d \Omega}(p, \theta)= & c_{1} p^{c_{2}}\left(1-\frac{p}{p_{B}-c_{9}}\right) \\
& \times \exp \left(-c_{3} \frac{p^{c_{4}}}{p_{B}^{c_{5}}}-c_{6} \theta\left(p-c_{7} p_{B} \cos ^{c_{8}} \theta\right)\right),
\end{aligned}
$$

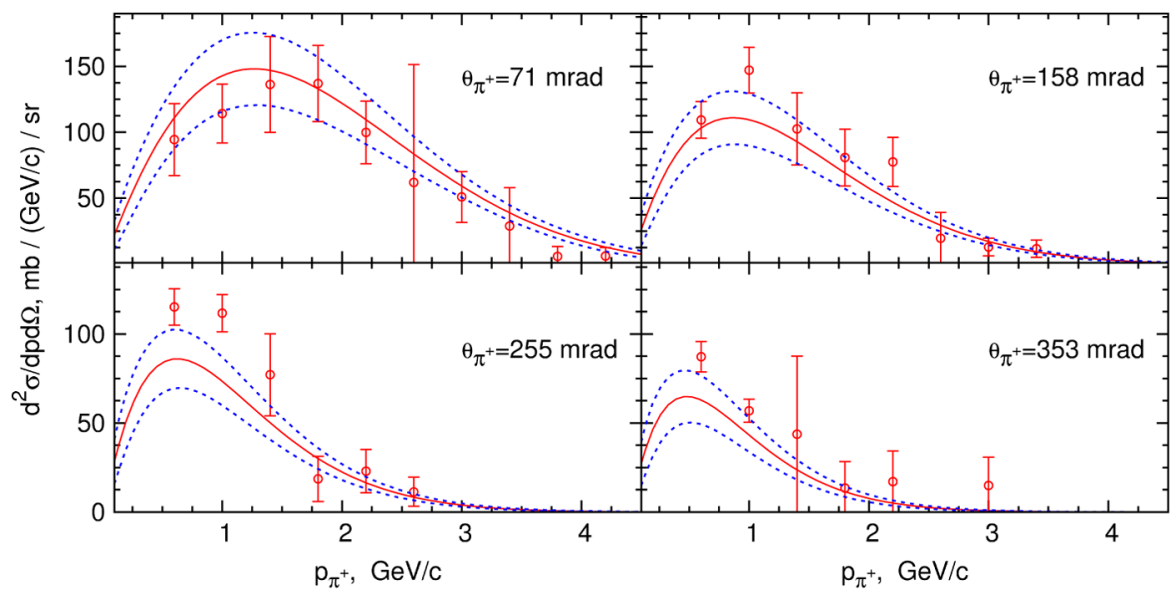

FIG. 20 (color online). Comparison of E910 $\pi^{+}$production cross section data [39] (circles) versus $p_{\pi}$ in bins of $\theta_{\pi}$ from $6.4 \mathrm{GeV} / c$ $p$-Be interactions and best-fit SW model (solid lines). The dashed lines represent the uncertainty band for $68 \%$ confidence level for 7 fit parameters. 


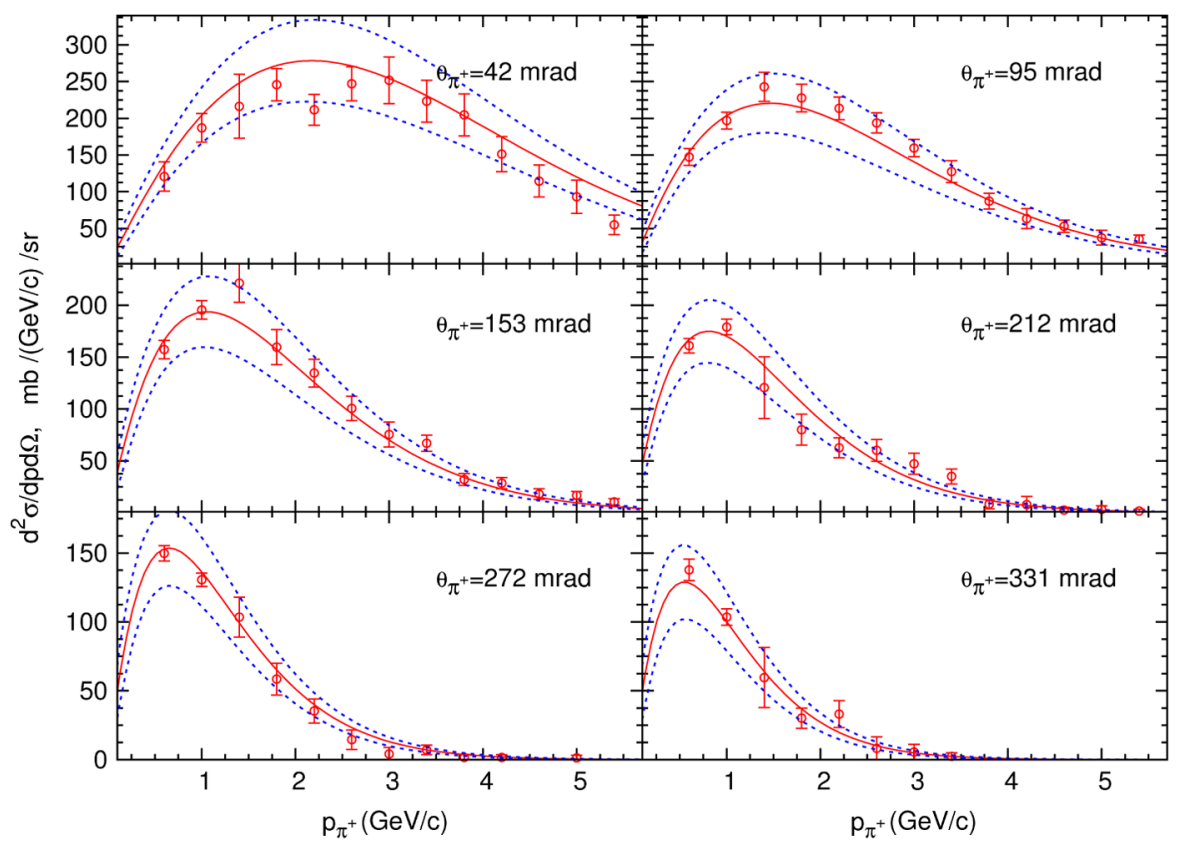

FIG. 21 (color online). Comparison of E910 $\pi^{+}$production cross section data [39] (circles) versus $p_{\pi}$ in bins of $\theta_{\pi}$ from $12.3 \mathrm{GeV} / c p$-Be interactions and best-fit SW model (solid lines). The dashed lines represent the uncertainty band resulting from varying the parameters within their correlated uncertainties, as described in the text.

where $\frac{d^{2} \sigma}{d p d \Omega}$ is the double differential cross section, $p$ is the total momentum of the meson in $\mathrm{GeV} / c, \theta$ is the angle of the meson with respect to the incident proton in radians, $p_{B}$ is the momentum of the incident proton in $\mathrm{GeV} / c$, and $c_{1}, \ldots, c_{9}$ are parameters to be determined in the fit to the production data. For the fits to the pion production data, $c_{9}$ is set to unity; for the kaon production fits (see below), it is a free parameter. The parametrization allows one to relate production data at different incident proton energies, to smoothly interpolate the behavior of the cross section

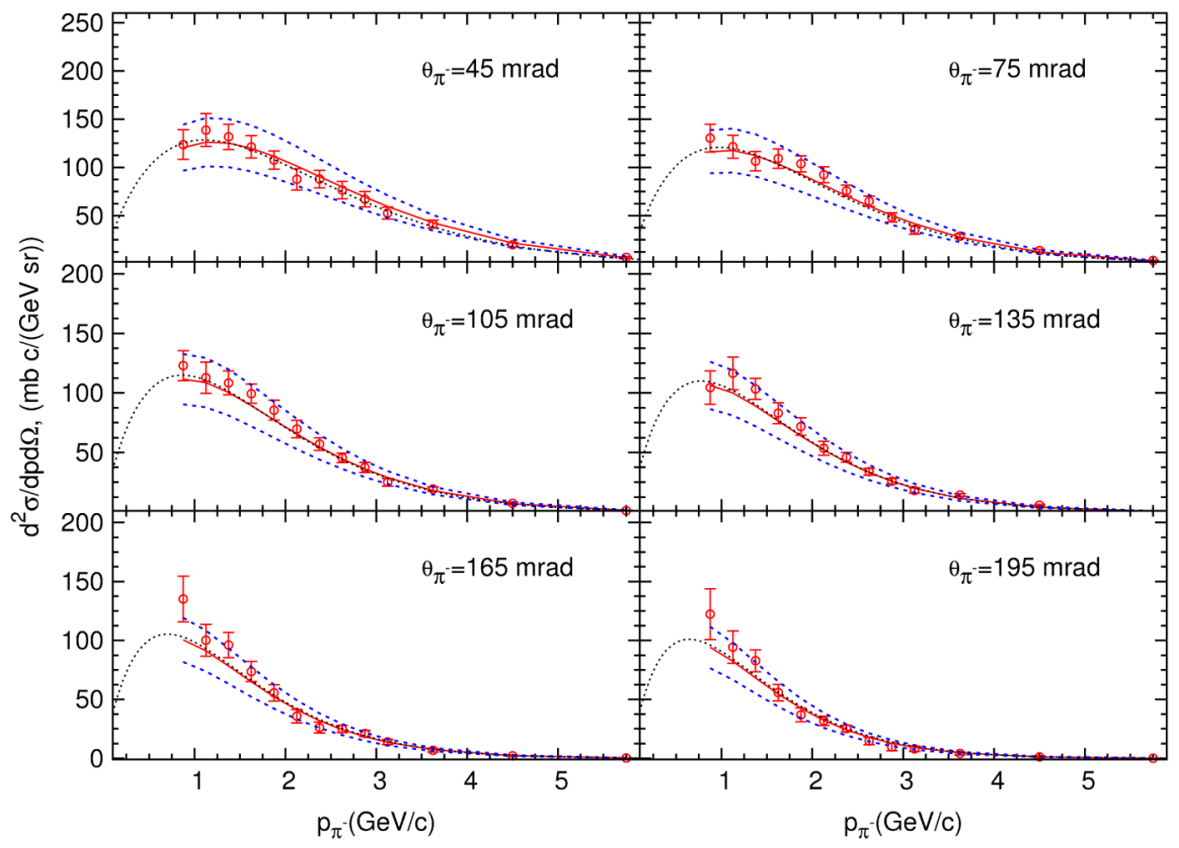

FIG. 22 (color online). Comparison of HARP $\pi^{-}$production cross section data [47] (circles) versus $p_{\pi}$ in bins of $\theta_{\pi}$ from $8.89 \mathrm{GeV} / c p$-Be interactions and best-fit SW model (solid lines). The dashed lines represent the uncertainty band resulting from varying the parameters within their correlated uncertainties, as described in the text. 


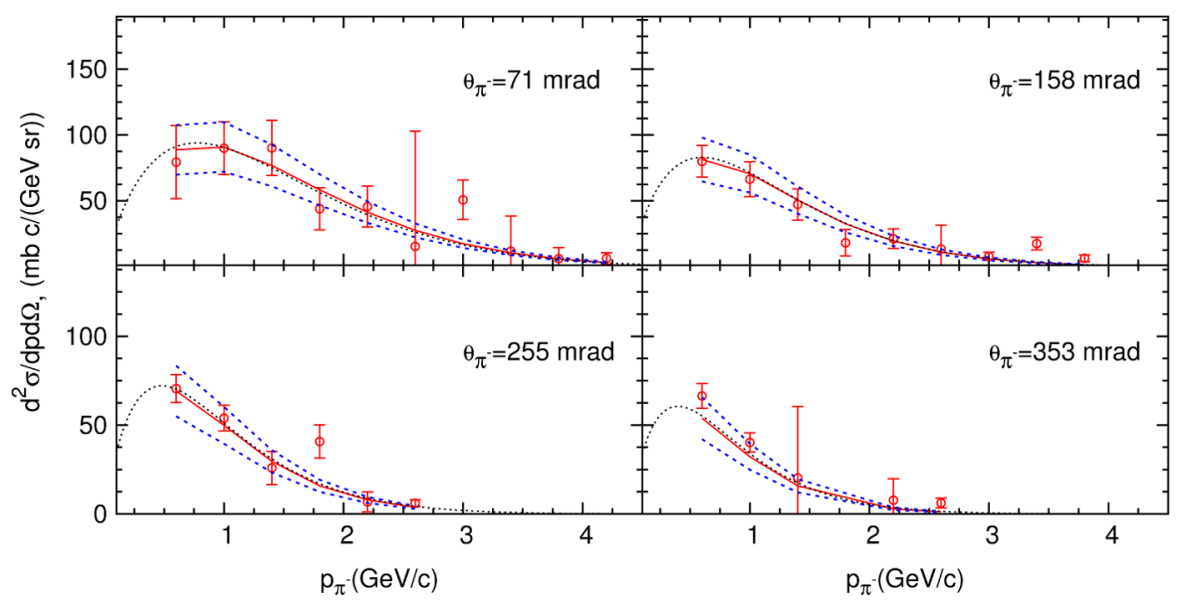

FIG. 23 (color online). Comparison of E910 $\pi^{-}$production cross section data [39] (circles) versus $p_{\pi}$ in bins of $\theta_{\pi}$ from $6.4 \mathrm{GeV} / c$ $p$-Be interactions and best-fit SW model (solid lines). The dashed lines represent the uncertainty band resulting from varying the parameters within their correlated uncertainties, as described in the text.

between measured points, and to extrapolate into regions where production data do not exist. Because of the strong correlation between the $c_{3}, c_{4}$, and $c_{5}$ parameters, the value of $c_{3}$ is fixed to unity for the $\pi^{+}$production fit. For $\pi^{-}$, the $c_{3}$ parameter is initially floating, but then fixed to its initial best-fit value when the fit is iterated. The correlation results from the limited range of proton momentum covered by the measurements $(6.4,8.9$ and $12.3 \mathrm{GeV} / c)$. As a result, the data has limited ability to constrain the cross section dependence on the proton momentum. The predicted pion production properties, however, are not affected by this indeterminacy.

The values of the parameters $c_{i}$ are determined from a fit to the $\pi^{ \pm}$production cross section data by minimizing the following $\chi^{2}$ function

$$
\begin{aligned}
\chi^{2}= & \sum_{k}\left[\left(\sum_{i, j}\left(D_{i, k}-N_{k} T_{i}\right) \mathbf{V}_{i j, k}^{-1}\left(D_{j, k}-N_{k} T_{j}\right)\right)\right. \\
& \left.+\frac{\left(N_{k}-1\right)^{2}}{\sigma_{k}^{2}}\right]
\end{aligned}
$$

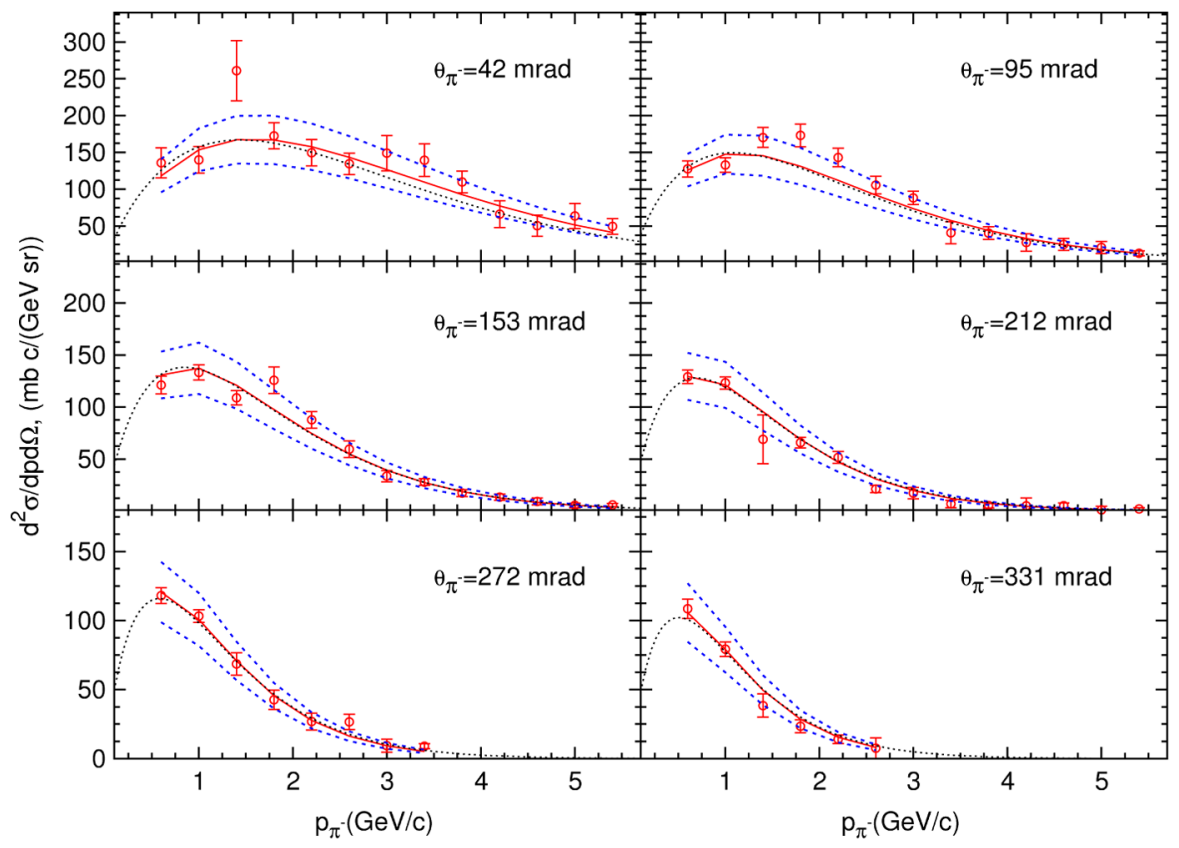

FIG. 24 (color online). Comparison of E910 $\pi^{-}$production cross section data [39] (circles) versus $p_{\pi}$ in bins of $\theta_{\pi}$ from $12.3 \mathrm{GeV} / c p$-Be interactions and best-fit SW model (solid line). The dashed lines represent the uncertainty band resulting from varying the parameters within their correlated uncertainties. 
TABLE V. Extracted Sanford-Wang parameters $c_{1-8}$ (first row), covariance matrix (upper right triangle including diagonals terms), and correlation coefficients (lower left triangle), for $\pi^{+}$secondary production in $p$-Be interactions. There are no entries in the covariance matrix for parameter $c_{3}$, which is fixed in the fit due to its large correlation with $c_{5}$.

\begin{tabular}{lcccccccc}
\hline \hline & $c_{1}$ & $c_{2}$ & $c_{3}$ & $c_{4}$ & $c_{5}$ & $c_{6}$ & $c_{7}$ & $c_{8}$ \\
Value & 220.7 & 1.080 & 1.000 & 1.978 & 1.32 & 5.572 & 0.0868 & 9.686 \\
\hline$c_{1}$ & 1707.2 & 1.146 & - & -17.646 & -15.968 & -8.81 & -0.7347 \\
$c_{2}$ & 0.139 & 0.0396 & - & -0.1072 & -0.0993 & 0.0325 & 0.0007 & -60.816 \\
$c_{3}$ & - & - & - & - & - & - & -0.0777 \\
$c_{4}$ & -0.554 & -0.699 & - & 0.5945 & 0.5049 & 0.0655 & 0.0025 \\
$c_{5}$ & -0.582 & -0.751 & - & 0.986 & 0.4411 & 0.0568 & 0.0025 \\
$c_{6}$ & -0.469 & 0.359 & - & 0.187 & 0.188 & 0.2066 & 0.0047 & 0.198 \\
$c_{7}$ & -0.795 & 0.157 & - & 0.145 & 0.168 & 0.462 & 0.0005 & 0.1031 \\
$c_{8}$ & -0.368 & -0.098 & - & 0.064 & 0.085 & 0.057 & 0.0641 \\
\hline \hline
\end{tabular}

where $D_{i, k}$ is the $i$-th data point for the $k$-th data set, $T_{i}$ is the value of the SW function for the kinematic parameters for that data point, $\mathbf{V}_{i j, k}$ is the bin-to-bin covariance matrix for the $k$-th data set, $N_{k}$ is relative normalization fit parameter for data set $k$ and $\sigma_{k}$ is the quoted normalization uncertainty for data set $k$. There are three data sets used in the fit, namely, HARP and E910 6.4 and $12.3 \mathrm{GeV} / c$. The normalization uncertainties for data sets at different beam momenta from the same experiment are the same and varied in common across these data sets during the fit procedure.

Since the data represent the average differential cross section over a range of angle and momentum ("bin"), bincentering corrections must be applied to the fit function. The bin-centering corrections are model-dependent since one must assume how the production cross section varies within a bin. Here, the SW parametrization is used to evaluate the bin-centering correction

$$
\mathrm{BCC}_{i j}=\frac{\mathrm{SW}\left(p_{i}^{c}, \theta_{j}^{c}\right)\left(\Delta p_{i} \Delta \cos \theta_{j}\right)}{\int_{p_{i}}^{p_{i+1}} \int_{\theta_{j}}^{\theta_{j+1}} \mathrm{SW}(p, \theta) \sin \theta d p d \theta},
$$

where $\left(p_{i}^{c}, \theta_{j}^{c}\right)$ is the center of bin $(i, j)$ in the $(p, \theta)$ space,
$\operatorname{SW}\left(p_{i}^{c}, \theta_{j}^{c}\right)$ is the double differential cross section returned by Eq. (11), and $\Delta p_{i}$ and $\Delta \cos \theta_{j}$ are the bin widths. The MINUIT fits are iterated with bin-centering corrections until convergence is achieved. Since we are concerned for the most part with pion production at $8.89 \mathrm{GeV} / c$ proton momentum, the dependence on the proton momentum is not important in predicting neutrino fluxes.

For the fit to the $\pi^{+}$data, the minimized $\chi^{2} /$ degree of freedom (DOF) using the reported experimental uncertainties is 1.8. To obtain parameter uncertainties, the fit is performed with the covariance matrices scaled by this factor, resulting in an effective $\chi^{2}$ of unity. The fit parameters are shown in the first row of Table V. The error matrix, shown below the parameters in Table $\mathrm{V}$, is obtained by varying the parameters in such a way that the resulting variations in the SW function cover the spread in the data points. This corresponds to an envelope of parameter variations in which the resulting $\chi^{2}$ is within 8.14 of the minimum determined by the fit. While this corresponds to a $68 \%$ confidence level parameter envelope for $7 \mathrm{pa}$ rameters, the $\chi^{2}$ difference is set by the desire to have the variations cover the deviations of the data points and their uncertainties. The normalization factors obtained from the two data sets also are compatible within the systematic

TABLE VI. Extracted Sanford-Wang parameters $c_{1-8}$ (first row), covariance matrix (upper right triangle including diagonals terms), and correlation coefficients (lower left triangle), for $\pi^{+}$secondary production in $p$-Be interactions. There are no entries in the covariance matrix for parameter $c_{3}$, which is fixed in the fit due to its large correlation with $c_{5}$.

\begin{tabular}{lcccccccc}
\hline \hline & $c_{1}$ & $c_{2}$ & $c_{3}$ & $c_{4}$ & $c_{5}$ & $c_{6}$ & $c_{7}$ & $c_{8}$ \\
Value & 213.7 & 0.9379 & 5.454 & 1.210 & 1.284 & 4.781 & 0.07338 & 8.329 \\
\hline$c_{1}$ & 3688.9 & 7.61 & - & -15.666 & -17.48 & -11.329 & -0.9925 & -91.4 \\
$c_{2}$ & 0.636 & 0.0388 & - & -0.0437 & -0.0509 & 0.0102 & -0.0009 & -0.1957 \\
$c_{3}$ & - & - & - & - & - & - & - & 0.0029 \\
$c_{4}$ & -0.889 & -0.765 & - & 0.0841 & 0.0895 & 0.0301 & 0.2588 \\
$c_{5}$ & -0.917 & -0.823 & - & 0.983 & 0.0986 & 0.0375 & 0.0033 \\
$c_{6}$ & -0.467 & 0.130 & - & 0.260 & 0.299 & 0.1595 & 0.0051 \\
$c_{7}$ & -0.731 & -0.204 & - & 0.447 & 0.470 & 0.571 & 0.0005 & 0.1933 \\
$c_{8}$ & -0.362 & -0.239 & - & 0.215 & 0.241 & 0.117 & 0.689 \\
\hline \hline
\end{tabular}


TABLE VII. Summary of $K^{+}$production measurements in $p$-Be interactions used to characterize $K^{+}$production in the BNB. The table includes $P_{\text {beam }}$, the primary proton momenta in the measurement, the momentum and angular ranges of the measurements, as well as the corresponding ranges of the Feynman-scaling variable $x_{F}$ and transverse momentum $p_{T}$. Finally, the quoted overall normalization uncertainty $\sigma_{N}$ is listed.

\begin{tabular}{lccccr}
\hline \hline Dataset & $P_{\text {beam }}(\mathrm{GeV} / c)$ & $P_{K^{+}}(\mathrm{GeV} / c)$ & $\theta_{K^{+}}($degrees $)$ & $x_{F}$ & $p_{T}(\mathrm{GeV} / c)$ \\
\hline Abbott [48] & 14.6 & $2-8$ & $20-30$ & $-0.12-0.07$ & $0.2-0.7$ \\
Aleshin [44] & 9.5 & $3-6.5$ & 3.5 & $0.3-0.8$ & $0.2-0.4$ \\
Allaby [49] & 19.2 & $3-16$ & $0-7$ & $0.3-0.9$ & $0.1-1.0$ \\
Dekkers [50] & $18.8,23.1$ & $4-12$ & 0,5 & $0.1-0.5$ & $0.0-1.2$ \\
Eichten [51] & 24.0 & $4-18$ & $0-6$ & $0.1-0.8$ & $0.1-1.2$ \\
Lundy [52] & 13.4 & $3-6$ & $2,4,8$ & $0.1-0.6$ & $20 \%$ \\
Marmer [53] & 12.3 & $0.5-1.0$ & $0,5,10$ & $-0.3-1.0$ & $0.1-1.2$ \\
Vorontsov [54] & 10.1 & $1-4.5$ & 3.5 & $0.03-0.5$ & $20 \%$ \\
\hline \hline
\end{tabular}

uncertainties quoted by the two experiments $\left(N_{\mathrm{HARP}}=\right.$ $\left.0.966, N_{\mathrm{E} 910}=1.048\right)$.

Likewise, the fit to the HARP [47] and E910 $\pi^{-}$production data with nominal errors resulted in a best-fit $\chi^{2}$ of 1.16/DOF. The experimental uncertainties are scaled by this factor to achieve a $\chi^{2} / \mathrm{DOF}$ of unity. The resulting parameters and covariance matrix are shown in Table VI, where the covariances are shown in the upper right triangle of the matrix (including the diagonal terms) and the correlation coefficients are shown in the lower left triangle of the matrix. The parametrizations using these best-fit parame- ters, along with the expected variation due to the parameter uncertainties, are shown along with the production data in Figs. 19-24.

\section{C. $K^{+}$production measurements}

For charged kaons, whose decays result in a significant contribution to the $\nu_{\mu}$ flux at high energies as well as the $\nu_{e}$ flux through the $K_{e 3}$ decay mode, there are no measurements from the HARP or BNL E910. As a result, measurements reported by other experiments measuring $\mathrm{K}^{+}$

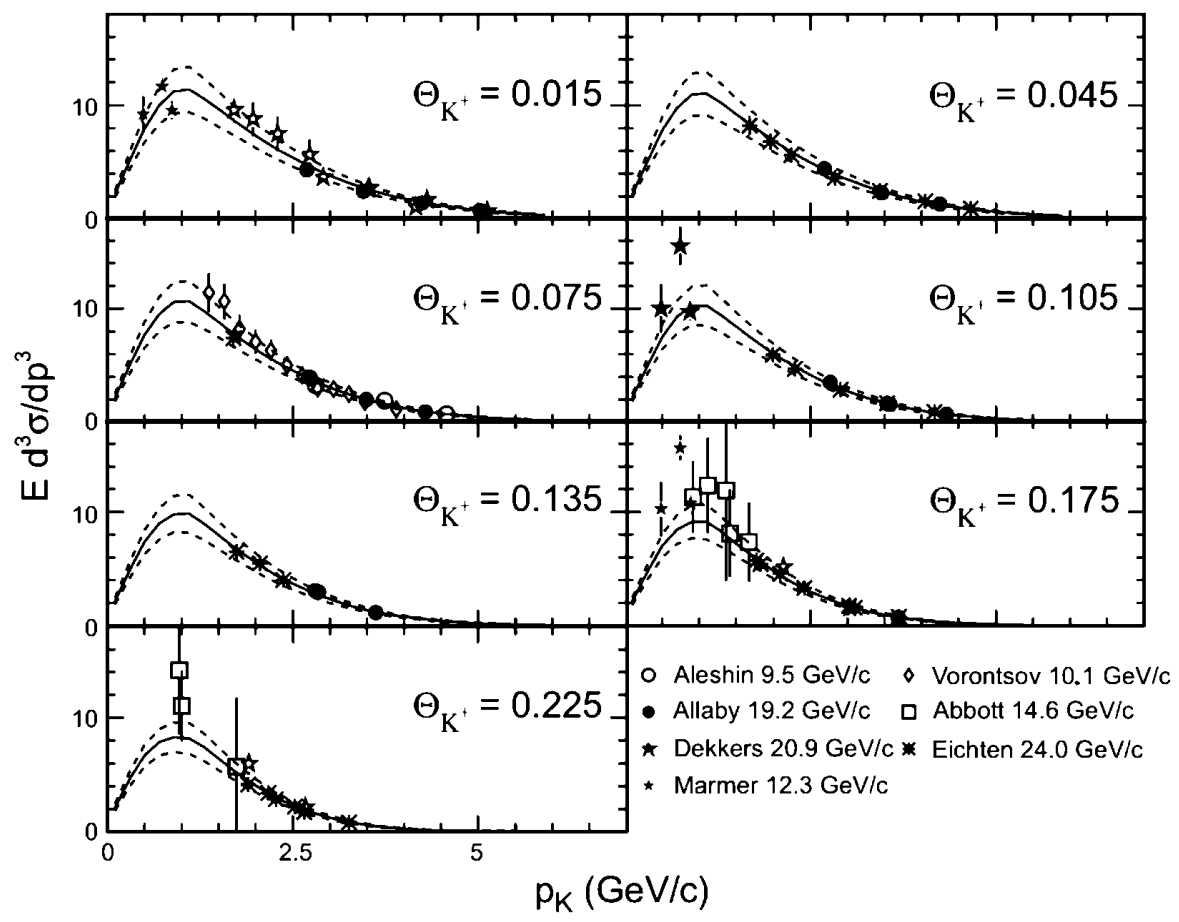

FIG. 25. Comparison of $K^{+}$invariant cross section data (points) as a function of $p_{K^{+}}$, the $K^{+}$momentum, in bins of $\Theta_{K^{+}}$, the $K^{+}$ production angle (in radians), with the Feynman-scaling based parametrization with best-fit parameters shown as a solid line. The scaling has been used to relate the measurements at different primary beam momenta to the $8.89 \mathrm{GeV} / c$ primary momentum in the BNB. Normalization factors from the best-fit are applied to the data. The dashed lines represent the uncertainty band resulting from varying the parameters within their correlated uncertainties. The uncertainty bands include the factor 1.5 error inflation to set $\chi^{2} / \mathrm{DOF}=1$. 
production in $p$-Be interactions at primary beam momenta close to $8.89 \mathrm{GeV} / c$ are used [44,48-54]. The measurements are summarized in Table VII.

Since no measurements of $K^{+}$exist at the $8.89 \mathrm{GeV} / c$ BNB primary momentum, we employ the Feynman scaling hypothesis to relate $K^{+}$production measurements at different primary energies to the expected production at $8.89 \mathrm{GeV} / c$. Theoretically, Feynman scaling should be a better model for comparing data from different primary beam momenta. This is born out by comparisons of data scaled to the BNB momentum of $8.89 \mathrm{GeV} / c$ as shown in Fig. 25. The hypothesis states that the invariant cross section is a function of only two variables, namely $x_{F}$ and $p_{T}$, where

$$
x_{F}=\frac{p_{\|}^{\mathrm{cm}}}{p_{\|}^{\max , \mathrm{cm}}}
$$

is the Feynman scaling variable, defined as the ratio of the parallel component of the momentum of the produced particle in the center-of-mass frame and the maximum possible value of this quantity for the given reaction, and $p_{T}$ is the transverse component of the momentum of the produced particle. In calculating $x_{F}$, the $p_{\|}^{\max , \mathrm{cm}}$ value is taken from the exclusive channel $p+(p / n) \rightarrow \Lambda^{0}+$ $(p / n)+K^{+}$. A more complete description of the Feynman scaling fit procedure and results can be found in Ref. [55].

The Feynman scaling is used as a basis for parametrizing the production data using the variables $x_{F}$ and $p_{T}$. This motivates a six-parameter model given by:

$$
\frac{d^{2} \sigma}{d p d \Omega}=\frac{p_{K^{+}}^{2}}{E_{K^{+}}}\left(E_{K^{+}} \frac{d^{3} \sigma}{d p_{K^{+}}^{3}}\right)=\frac{p_{K^{+}}^{2}}{E_{K^{+}}} \times c_{1}\left(1-\left|x_{F}\right|\right) \exp \left[-c_{2} p_{T}-c_{3}\left|x_{F}\right|^{c_{4}}-c_{5} p_{T}^{2}-c_{7}\left|p_{T} \times x_{F}\right|^{c_{6}}\right]
$$

The model is basically a translation of the Feynman scaling hypothesis where the invariant cross section is only a function of $x_{F}$ and $p_{T}$. It incorporates an exponentially falling $p_{T}$ distribution, correlations between $p_{T}$ and $x_{F}$, a flat rapidity plateau at $x_{F}=0$ and zero cross section as $x_{F} \rightarrow 1$. The kinematic threshold constraint is imposed by setting the function equal to zero for $\left|x_{F}\right|>1$. Figure 25 shows the momentum distribution of the data scaled to $8.89 \mathrm{GeV} / c$ primary momentum in bins of scaled $K^{+}$ production angle. The data include normalizations factors obtained from the fit procedure described below. The right plot of Fig. 18 shows as boxes the $x_{F}$ versus $p_{T}$ distribution of $K^{+}$, produced in the target that produce neutrinos at the MiniBooNE detector. The colored points indicate the kinematic coverage of the various measurements in these two variables.
The $c_{i}$ parameters are determined in a $\chi^{2}$ fit to the production data for $1.2<p_{K^{+}}^{\mathrm{BNB}}<5.5$, where $p_{K^{+}}^{\mathrm{BNB}}$ is the kaon momentum translated to the BNB primary energy using Feynman scaling. The $p_{K^{+}}^{\mathrm{BNB}}$ requirement eliminates most of the data at negative $x_{F}$, where nuclear effects are expected to be dominant. The $\chi^{2}$ takes the same form as in Eq. (12), where the covariance matrix from the experiments is diagonal, and the quoted normalization uncertainties are used to constrain the normalization factors. The Vorontsov data [54] has indications of an error in the normalization outside of their quoted uncertainties. As a result, a large normalization uncertainty $(500 \%)$ was assigned to these measurements with the effect that the measurements from this experiment contributes only "shape" information without any normalization constraint. The discrepancy is not apparent in Fig. 25 since

TABLE VIII. Best-fit Feynman-scaling model parameters $c_{i}$ from a fit to $K^{+}$production data (first row). The covariance matrix for the parameters with uncertainties inflated by a factor of 1.5 to set $\chi^{2} / \mathrm{DOF}=1$ is in the upper right triangle (including diagonal terms) of the table below the parameters, but does not include the additional inflation by a factor of 4 described in the text. The lower left

\begin{tabular}{|c|c|c|c|c|c|c|c|}
\hline Value & $\begin{array}{c}c_{1} \\
11.70\end{array}$ & $\begin{array}{c}c_{2} \\
0.88\end{array}$ & $\begin{array}{c}c_{3} \\
4.77\end{array}$ & $\begin{array}{c}c_{4} \\
1.51\end{array}$ & $\begin{array}{c}c_{5} \\
2.21\end{array}$ & $\begin{array}{c}c_{6} \\
2.17\end{array}$ & $\begin{array}{c}c_{7} \\
1.51\end{array}$ \\
\hline$c_{1}$ & 1.094 & 0.0502 & $2.99 \cdot 10^{-3}$ & -0.0332 & -0.0375 & 0.125 & 0.0743 \\
\hline$c_{2}$ & 0.378 & 0.0161 & $1.39 \cdot 10^{-3}$ & $-1.44 \cdot 10^{-3}$ & -0.0126 & 0.0322 & 0.022 \\
\hline$c_{3}$ & 0.033 & 0.127 & $7.47 \cdot 10^{-3}$ & $2.06 \cdot 10^{-3}$ & $1.93 \cdot 10^{-3}$ & 0.0135 & $-3.34 \cdot 10^{-3}$ \\
\hline$c_{4}$ & -0.540 & -0.193 & 0.405 & $3.46 \cdot 10^{-3}$ & $2.03 \cdot 10^{-3}$ & $-4.11 \cdot 10^{-3}$ & $-6.28 \cdot 10^{-3}$ \\
\hline$c_{5}$ & -0.297 & -0.822 & 0.185 & 0.286 & 0.0146 & -0.0154 & -0.0244 \\
\hline$c_{6}$ & 0.280 & 0.595 & 0.366 & -0.164 & -0.299 & 0.182 & 0.126 \\
\hline$c_{7}$ & 0.178 & 0.435 & -0.097 & -0.268 & -0.506 & 0.741 & 0.159 \\
\hline
\end{tabular}
triangle shows the correlation coefficients. 


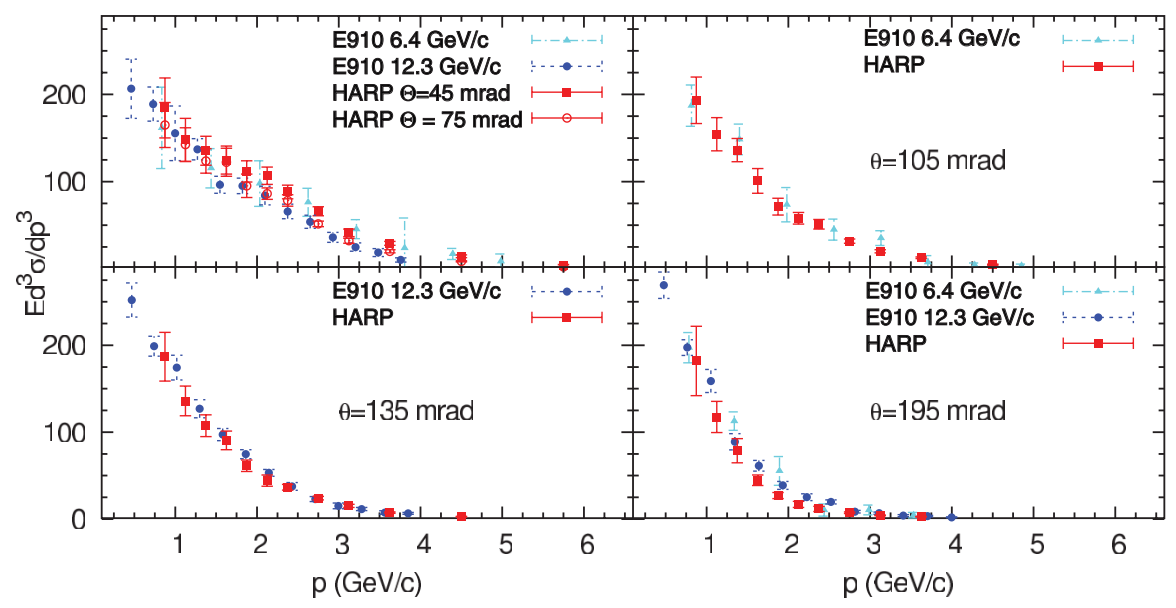

FIG. 26 (color). Invariant pion production cross section from HARP and E910 versus $p_{\pi}$ in bins of $\theta_{\pi}$. The E910 measurements are rescaled to $p_{B}=8.89 \mathrm{GeV} / c$.

the fitted normalization factor is applied to the measured cross sections. The Lundy data [52] were excluded from the fit due to inconsistencies with the other measurements, while the Marmer measurements [53] were excluded by the $p_{K^{+}}^{\mathrm{BNB}}$ requirement.

The $\chi^{2} / \mathrm{DOF}$ for the fit is 2.28 . The errors in the measurements are inflated by a factor of $\sqrt{2.28}=1.5$ to bring the $\chi^{2} / \mathrm{DOF}$ to unity. The covariance matrix of the parameters is extracted in the same way as employed for the pion production fits. Table VIII summarizes the results, with the first row listing the seven best-fit parameters, and the $7 \times 7$ matrix below it listing the covariance. While the parametrization represents the measurements quite well (as shown in Fig. 25), the uncertainties are inflated by a further factor of 4 for the $\nu_{\mu} \rightarrow \nu_{e}$ oscillation analysis to account for inconsistencies within the production data, the use of the Feynman-scaling hypothesis to relate the production measurements from experiments with proton momenta different from the $8.89 \mathrm{GeV} / c$ used at the BNB, and a possible discrepancy in the rate of $\nu_{\mu}$ events observed in the MiniBooNE detector compared with the predictions based on the beam line simulation [3]. The covariance matrix in Table VIII and the uncertainty bands in Fig. 25 do not include this further inflation of the uncertainties by a factor of 4 .

Figure 26 shows a similar comparison of the E910 and HARP $\pi^{+}$production data where the E910 data have been scaled to $8.89 \mathrm{GeV} / c$ primary momentum. The data indicates that the $\pi^{+}$production is also consistent with the Feynman scaling hypothesis. When fit to Eq. (15), a slightly poorer $\chi^{2}$ results than in the SW fit. As a result, the Feynman scaling model is not used in the $\pi^{+}$production model.

\section{Production of $K^{\mathbf{0}}, K^{-}$and other particles}

A scheme similar to that used to parametrize the $\pi^{+}$and $K^{+}$production data is used for neutral kaon production, for which the $K_{e 3}$ decay mode of the $K_{L}^{0}$ is a source of background $\nu_{e}$. Since the kaons are produced in strong interactions as $K^{0}$ and $\bar{K}^{0}$ (primarily the former), the kaons

TABLE IX. Best-fit Sanford-Wang model parameters $c_{i}$ from a fit to $K_{S}^{0}$ production data (first row). The covariance matrix for the parameters is in the upper right triangle (including diagonal terms) of the table below the parameters, while the bottom left corner of the table reports the corresponding correlation coefficients.

\begin{tabular}{lccccccccc}
\hline \hline & $c_{1}$ & $c_{2}$ & $c_{3}$ & $c_{4}$ & $c_{5}$ & $c_{6}$ & $c_{7}$ & $c_{8}$ & $c_{9}$ \\
Value & 15.130 & 1.975 & 4.084 & 0.928 & 0.731 & 4.362 & 0.048 & 13.300 & 1.278 \\
\hline$c_{1}$ & 32.3 & -0.09687 & 0.8215 & -0.1018 & -0.2124 & -0.8902 & -0.1333 & 16.55 & -1.789 \\
$c_{2}$ & -0.055 & 0.09574 & 0.03248 & 0.00131 & -0.01303 & 0.08836 & -0.00031 & -1.536 & -0.2156 \\
$c_{3}$ & 0.199 & 0.144 & 0.5283 & -0.01922 & 0.02267 & -0.0033 & -0.00236 & 0.0391 & -0.08017 \\
$c_{4}$ & -0.062 & 0.015 & -0.091 & 0.08442 & 0.00405 & 0.00071 & -0.00037 & -0.01443 & -0.07301 \\
$c_{5}$ & -0.377 & -0.425 & 0.315 & 0.141 & 0.00982 & 0.00287 & 0.00028 & -0.05777 & 0.02966 \\
$c_{6}$ & -0.261 & 0.476 & -0.008 & 0.004 & 0.048 & 0.3599 & 0.00385 & -4.751 & -0.1577 \\
$c_{7}$ & -0.724 & -0.031 & -0.100 & -0.039 & 0.087 & 0.198 & 0.00105 & 0.05806 & 0.00686 \\
$c_{8}$ & 0.255 & -0.435 & 0.005 & -0.004 & -0.051 & -0.694 & 0.157 & 130.2 & 1.222 \\
$c_{9}$ & -0.183 & -0.406 & -0.064 & -0.146 & 0.174 & -0.153 & 0.123 & 0.062 & 2.948 \\
\hline \hline
\end{tabular}


NEUTRINO FLUX PREDICTION AT MiniBooNE

TABLE X. Average multiplicity per particle-producing reaction for secondary particles produced in the inelastic collisions of $8.89 \mathrm{GeV} / c$ primary protons on beryllium, as well as average momentum $\langle p\rangle$ and angle $\langle\theta\rangle$ with respect to the primary proton direction. Multiplicities and average kinematics refer to particles produced in the forward hemisphere in the laboratory frame and with transverse momentum less than $1 \mathrm{GeV} / c$. *see comment in text.

\begin{tabular}{lccc}
\hline \hline Particle & $\begin{array}{c}\text { Multiplicity } \\
\text { per reaction }\end{array}$ & $\begin{array}{c}\langle p\rangle \\
(\mathrm{GeV} / c)\end{array}$ & $\begin{array}{c}\langle\theta\rangle \\
(\mathrm{mrad})\end{array}$ \\
\hline$p$ & 1.5462 & 2.64 & 441 \\
$n$ & 1.3434 & 1.59 & 586 \\
$\pi^{-}$ & $0.9004^{*}$ & 0.82 & 556 \\
$\pi^{+}$ & $0.8825^{*}$ & 1.11 & 412 \\
$K^{+}$ & 0.0689 & 1.69 & 332 \\
$K^{0}$ & 0.0241 & 1.34 & 414 \\
$K^{-}$ & 0.0024 & 1.26 & 259 \\
Total & 4.7679 & 1.69 & 496 \\
\hline \hline
\end{tabular}

have equal content as $K_{S}^{0}$ and $K_{L}^{0}$. As a result, the production properties of neutral kaons decaying as $K_{L}^{0}$ can be obtained by measuring the $K_{S}^{0}$ production properties. While the $K_{S}^{0}$ can contribute to the neutrino flux via the decay of the charged pions produced in the $K_{S}^{0} \rightarrow \pi^{+}+\pi^{-}$decay, the most important consideration is the production of $\nu_{e}$ from the decay of the $K_{L}^{0}$. The long life time of the $K_{L}^{0}$, together with the fact that they are not focussed, lead to the expectation that the contribution of neutrinos for this source will be small relative to the $K^{+}$.

The primary source of data for the parametrization comes from two measurements of $K_{S}^{0}$ production in $p$-Be interactions in the BNL E910 experiment $\left(p_{\text {beam }}=12.3\right.$ and $17.5 \mathrm{GeV} / c)$ and the measurements of Abe et al. [56] $\left(p_{\text {beam }}=12.3 \mathrm{GeV} / c\right)$ at KEK. Since the neutral kaons are not focused by the magnetic field of the horn, the forward production $\left(<5^{\circ}\right)$ is particularly relevant for predicting the BNB neutrino flux. While the production data from the BNL E910 and KEK measurements do not cover this region, the combination of the two data sets are sufficient to constrain the production cross section in this forward region via the Sanford-Wang parametrization. The extracted parameter values and covariance matrix are summarized in Table IX.

For $K^{-}$production, the scarcity of production measurements in the relevant kinematic regions motivated the use of the MARS hadronic interaction package [57] to determine the absolute double differential cross sections. The cross sections are obtained by simulating $8.89 \mathrm{GeV} / c$ $p$-Be interactions on a thin beryllium target and recording the rate and spectrum of outgoing $K^{-}$. The expected relative contribution of neutrinos of all species from $K^{-}$ decays is expected to be small. Neutrino flux contributions from semileptonic hyperon decays (e.g. $\Lambda$, $\Sigma$, etc.), estimated using a FLUKA [58] simulation, are also negligible.
PHYSICAL REVIEW D 79, 072002 (2009)

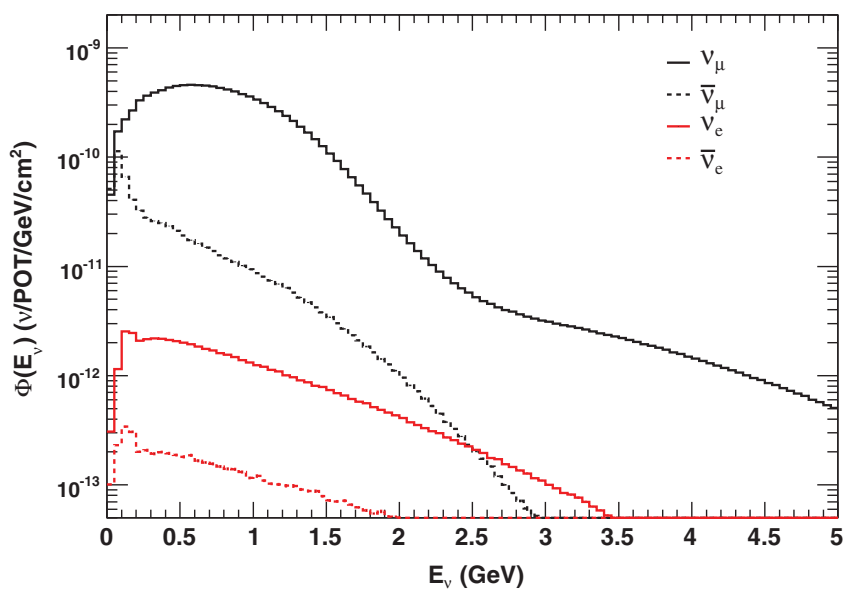

FIG. 27 (color). Total predicted flux at the MiniBooNE detector by neutrino species with horn in neutrino mode.

Secondary protons and neutrons emerging from the $p$-Be inelastic interactions are simulated based on the predictions of the MARS model, with the exception of quasielastic scattering, in which case the final state proton kinematics are handled by a custom model. The production of all other particle species is handled by the default Geant4 hadronic model.

The properties of the particle production model are summarized in Table $\mathrm{X}$. The table shows the average multiplicity per $p$-Be reaction (defined as inelastic interactions excluding quasielastic scattering), along with the mean momentum and production angle. The $\pi^{+}$and $\pi^{-}$ production occur with similar multiplicities, though the former tends to be harder and more forward directed. The larger overall multiplicity for the $\pi^{-}$is due to the extrapolation of the cross sections to large angles that are not covered in the HARP and E910 measurements. Since the contribution to the neutrino flux from such pions is small, the impact of uncertainty in this extrapolation is suppressed. The kaon production is an order of magnitude

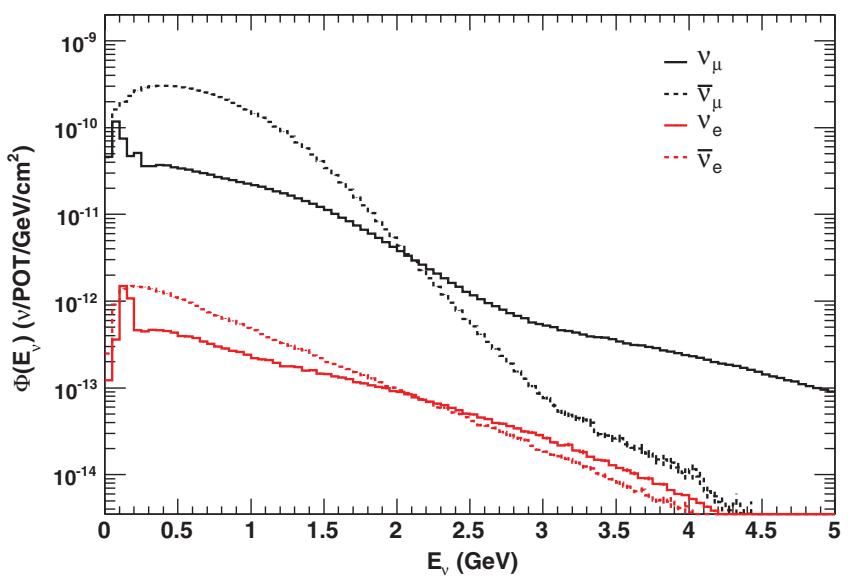

FIG. 28 (color). Total predicted flux at the MiniBooNE detector by neutrino species with horn in anti-neutrino mode. 
TABLE XI. Predicted $\nu_{\mu} / \bar{\nu}_{\mu}$ (top) and $\nu_{e} / \bar{\nu}_{e}$ (bottom) fluxes at the MiniBooNE detector with horn in neutrino mode. The contribution of flux from meson decays where the parent particle in the decay chain is produced by proton or neutron interaction. The "other" category includes channels with contributions less than those shown, along with cases where the parent particle in the decay chain is produced by a meson interaction.

\begin{tabular}{|c|c|c|c|c|}
\hline & \multicolumn{2}{|c|}{$\nu_{\mu}$} & \multicolumn{2}{|c|}{$\bar{\nu}_{\mu}$} \\
\hline Flux $\left(\nu / \mathrm{cm}^{2} /\right.$ POT $)$ & & $5.19 \times 10^{-10}$ & & $3.26 \times 10^{-11}$ \\
\hline Frac. of Total & & $93.6 \%$ & & $5.86 \%$ \\
\hline \multirow[t]{9}{*}{ Composition } & $\pi^{+}:$ & $96.72 \%$ & $\pi^{-}:$ & $89.74 \%$ \\
\hline & $K^{+}:$ & $2.65 \%$ & $\pi^{+} \rightarrow \mu^{+}:$ & $4.54 \%$ \\
\hline & $K^{+} \rightarrow \pi^{+}:$ & $0.26 \%$ & $K^{-}:$ & $0.51 \%$ \\
\hline & $K^{0} \rightarrow \pi^{+}:$ & $0.04 \%$ & $K^{0}:$ & $0.44 \%$ \\
\hline & $K^{0}:$ & $0.03 \%$ & $K^{0} \rightarrow \pi^{-}:$ & $0.24 \%$ \\
\hline & $\pi^{-} \rightarrow \mu^{-}:$ & $0.01 \%$ & $K^{+} \rightarrow \mu^{+}:$ & $0.06 \%$ \\
\hline & Other: & $0.30 \%$ & $K^{-} \rightarrow \pi^{-}:$ & $0.03 \%$ \\
\hline & & & Other: & $4.43 \%$ \\
\hline & \multicolumn{2}{|c|}{$\nu_{e}$} & \multicolumn{2}{|c|}{$\bar{\nu}_{e}$} \\
\hline Flux $\left(\nu / \mathrm{cm}^{2} /\right.$ POT $)$ & & $2.87 \times 10^{-12}$ & & $3.00 \times 10^{-13}$ \\
\hline Frac. of Total & & $0.52 \%$ & & $0.05 \%$ \\
\hline \multirow[t]{6}{*}{ Composition } & $\pi^{+} \rightarrow \mu^{+}:$ & $51.64 \%$ & $K_{L}^{0}:$ & $70.65 \%$ \\
\hline & $K^{+}:$ & $37.28 \%$ & $\pi^{-} \stackrel{L}{\rightarrow} \mu^{-}$ & $19.33 \%$ \\
\hline & $K_{L}^{0}:$ & $7.39 \%$ & $K^{-}:$ & $4.07 \%$ \\
\hline & $\pi^{+}:$ & $2.16 \%$ & $\pi^{-}:$ & $1.26 \%$ \\
\hline & $K^{+} \rightarrow \mu^{+}:$ & $0.69 \%$ & $K^{-} \rightarrow \mu^{-}:$ & $0.07 \%$ \\
\hline & Other: & $0.84 \%$ & Other: & $4.62 \%$ \\
\hline
\end{tabular}

TABLE XII. Predicted $\nu_{\mu} / \bar{\nu}_{\mu}$ (top) and $\nu_{e} / \bar{\nu}_{e}$ (bottom) fluxes at the MiniBooNE detector with horn in anti-neutrino mode. The contribution of flux from meson decays where the parent particle in the decay chain is produced by proton or neutron interaction. The other category includes channels with contributions less than those shown, along with cases where the parent particle in the decay chain is produced by a meson interaction.

\begin{tabular}{|c|c|c|c|c|}
\hline & \multicolumn{2}{|c|}{$\nu_{\mu}$} & \multicolumn{2}{|c|}{$\bar{\nu}_{\mu}$} \\
\hline Flux $\left(\nu / \mathrm{cm}^{2} /\right.$ POT $)$ & & $5.42 \times 10^{-11}$ & & $2.93 \times 10^{-10}$ \\
\hline Frac. of Total & & $15.71 \%$ & & $83.73 \%$ \\
\hline \multirow[t]{8}{*}{ Composition } & $\pi^{+}:$ & $88.79 \%$ & $\pi^{-}:$ & $98.4 \%$ \\
\hline & $K^{+}:$ & $7.53 \%$ & $K^{-}:$ & $0.18 \%$ \\
\hline & $\pi^{-} \rightarrow \mu^{-}:$ & $1.77 \%$ & $K^{0} \rightarrow \pi^{-}:$ & $0.05 \%$ \\
\hline & $K^{0}:$ & $0.26 \%$ & $K^{0}:$ & $0.05 \%$ \\
\hline & Other: & $2.00 \%$ & $\pi^{+} \rightarrow \mu^{+}:$ & $0.03 \%$ \\
\hline & & & $K^{-} \rightarrow \pi^{-}:$ & $0.02 \%$ \\
\hline & & & Other: & $1.30 \%$ \\
\hline & \multicolumn{2}{|c|}{$\nu_{e}$} & \multicolumn{2}{|c|}{$\bar{\nu}_{e}$} \\
\hline Flux $\left(\nu / \mathrm{cm}^{2} /\right.$ POT $)$ & & $6.71 \times 10^{-13}$ & & $1.27 \times 10^{-12}$ \\
\hline Frac. of Total & & $0.2 \%$ & & $0.4 \%$ \\
\hline \multirow[t]{6}{*}{ Composition } & $K^{+}:$ & $51.72 \%$ & $\pi^{-} \rightarrow \mu^{-}:$ & $75.67 \%$ \\
\hline & $K^{0}:$ & $31.56 \%$ & $K^{0}:$ & $16.51 \%$ \\
\hline & $\pi^{+} \rightarrow \mu^{+}$ & $13.30 \%$ & $K^{-}:$ & $3.08 \%$ \\
\hline & $\pi^{+}:$ & $0.83 \%$ & $\pi^{-}:$ & $2.58 \%$ \\
\hline & $K^{+} \rightarrow \mu^{+}:$ & $0.41 \%$ & $K^{-} \rightarrow \mu^{-}:$ & $0.06 \%$ \\
\hline & Other: & $2.17 \%$ & Other & $2.10 \%$ \\
\hline
\end{tabular}



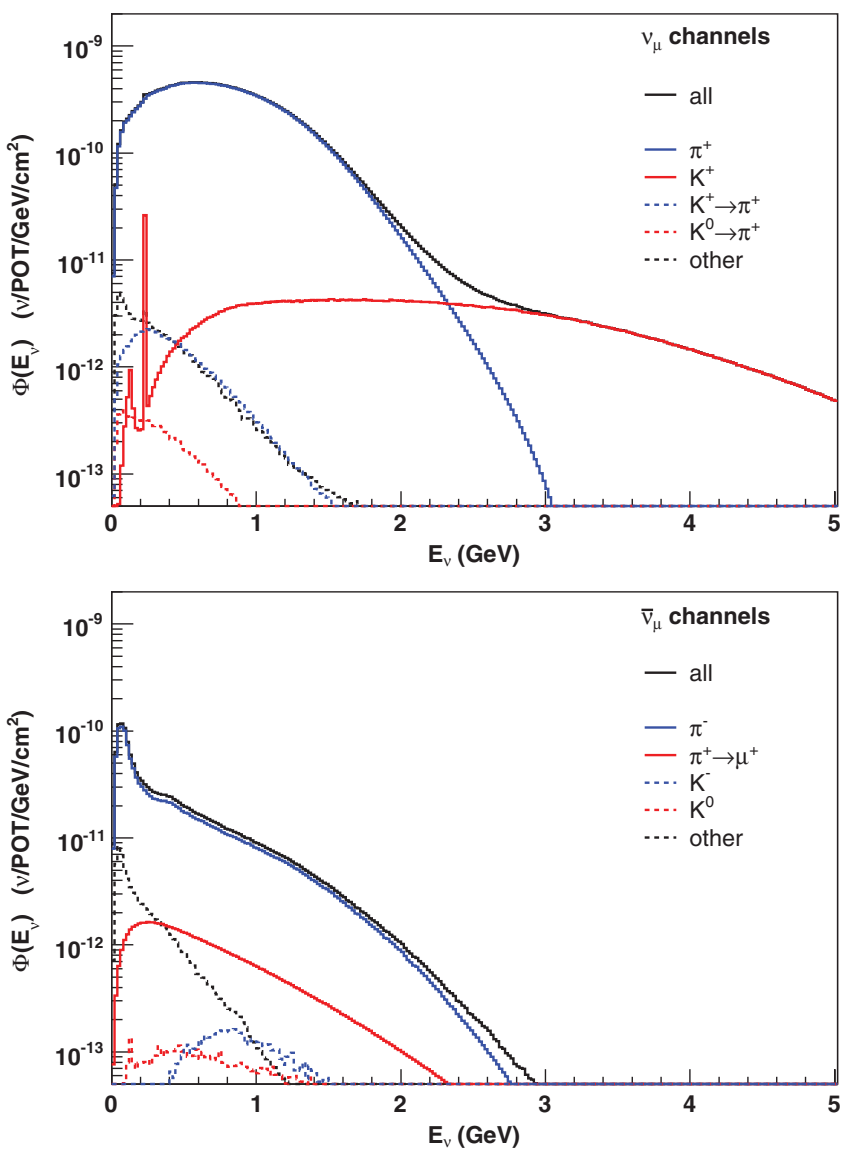

FIG. 29 (color). Predicted $\nu_{\mu}$ (top) and $\bar{\nu}_{\mu}$ (bottom) fluxes at the MiniBooNE detector by parent meson species with horn in neutrino mode. The black line is the total predicted flux, while all the subcomponents apart from the dashed black are from nucleon-induced meson production of the indicated decay chains. The dashed black histogram includes all other contributions, primarily from meson decay chains initiated by mesonnucleus interactions.

smaller than the pion production, with $K^{-}$production particularly suppressed relative to $K^{+}$and $K^{0}$ production.

\section{PREDICTION OF NEUTRINO FLUX AT MINIBOONE}

The results of the simulation are summarized in Figs. 27 and 28 , which show the total predicted flux of each neutrino species at the MiniBooNE detector in neutrino mode and antineutrino modes, respectively. In each case, the $\nu_{e} / \bar{\nu}_{e}$ contribution is less than $1 \%$ at the peak of the $\nu_{\mu} / \bar{\nu}_{\mu}$ flux, though it rises at higher energies. As shown, the predicted fluxes exhibit many features that are better understood by analyzing the sources of each component of the flux.

The integrated contribution of each (anti-)neutrino species, along with their dominant decay chains, are shown in Table XI for the neutrino-mode horn configuration, and
Table XII for the anti-neutrino-mode horn configuration. The dominant contribution from decay chains in which the parent meson is produced by a nucleon is separated from those in which it is produced by a meson interaction. This is due to the qualitatively different level of systematic understanding for the two processes. For the former, the production cross sections are based on the particle production experiments described in Sec. V, with systematic uncertainties propagated from the uncertainties reported by these experiments. For the latter, the simulation relies on the default Geant4 hadronic interaction model to provide the production cross sections. Fortunately, the latter is a small contribution to the flux in all cases.

Figure 29 shows the channels through which the $\nu_{\mu}$ and $\bar{\nu}_{\mu}$ are produced in neutrino mode. For the $\nu_{\mu}$ flux, the $\pi^{+} \rightarrow \nu_{\mu}$ contribution is dominant for energies less than $2 \mathrm{GeV}$, while the $K^{+} \rightarrow \nu_{\mu}$ flux become dominant at higher energies. The two peaks in the $K^{+}$flux at low
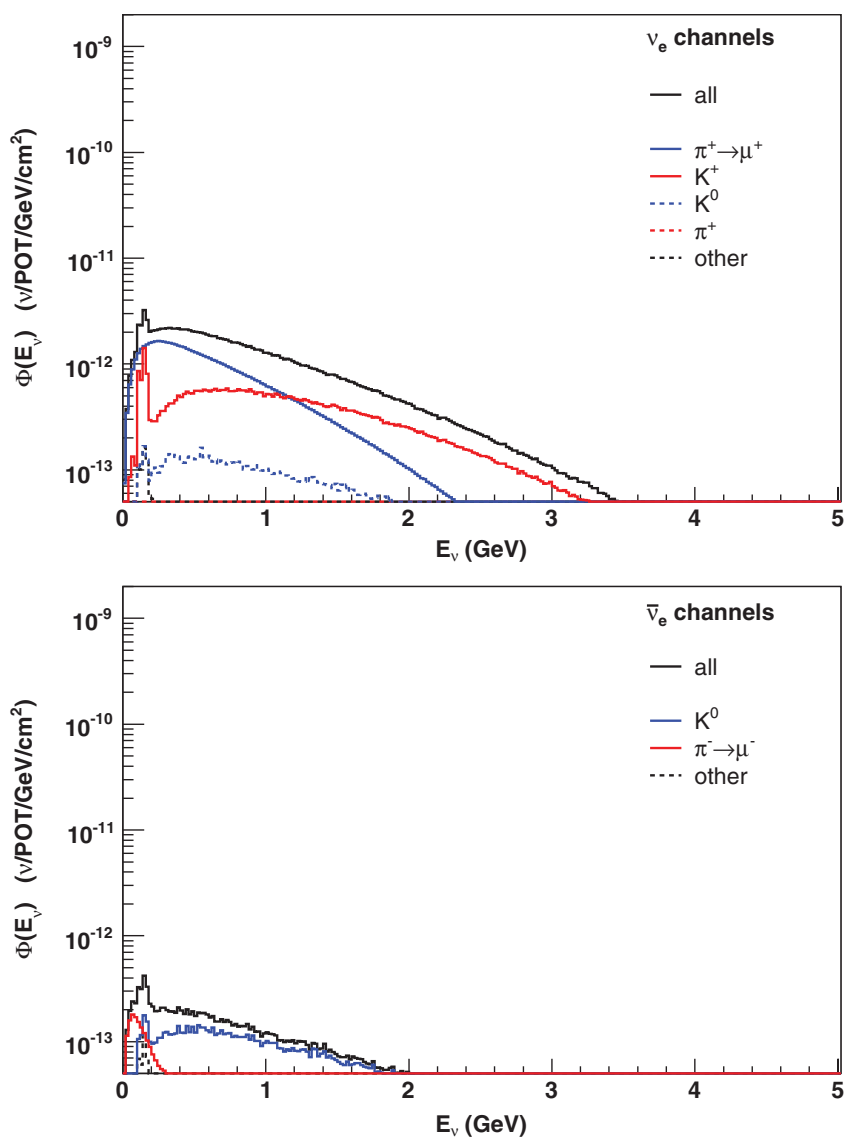

FIG. 30 (color). Predicted $\nu_{e}$ (top) and $\bar{\nu}_{e}$ (bottom) flux at the MiniBooNE detector by parent meson species with horn in neutrino mode. The black line is the total predicted flux, while all the subcomponents apart from the dashed black are from nucleon-induced meson production of the indicated decay chains. The dashed black histogram includes all other contributions, primarily from meson decay chains initiated by mesonnucleus interactions. 

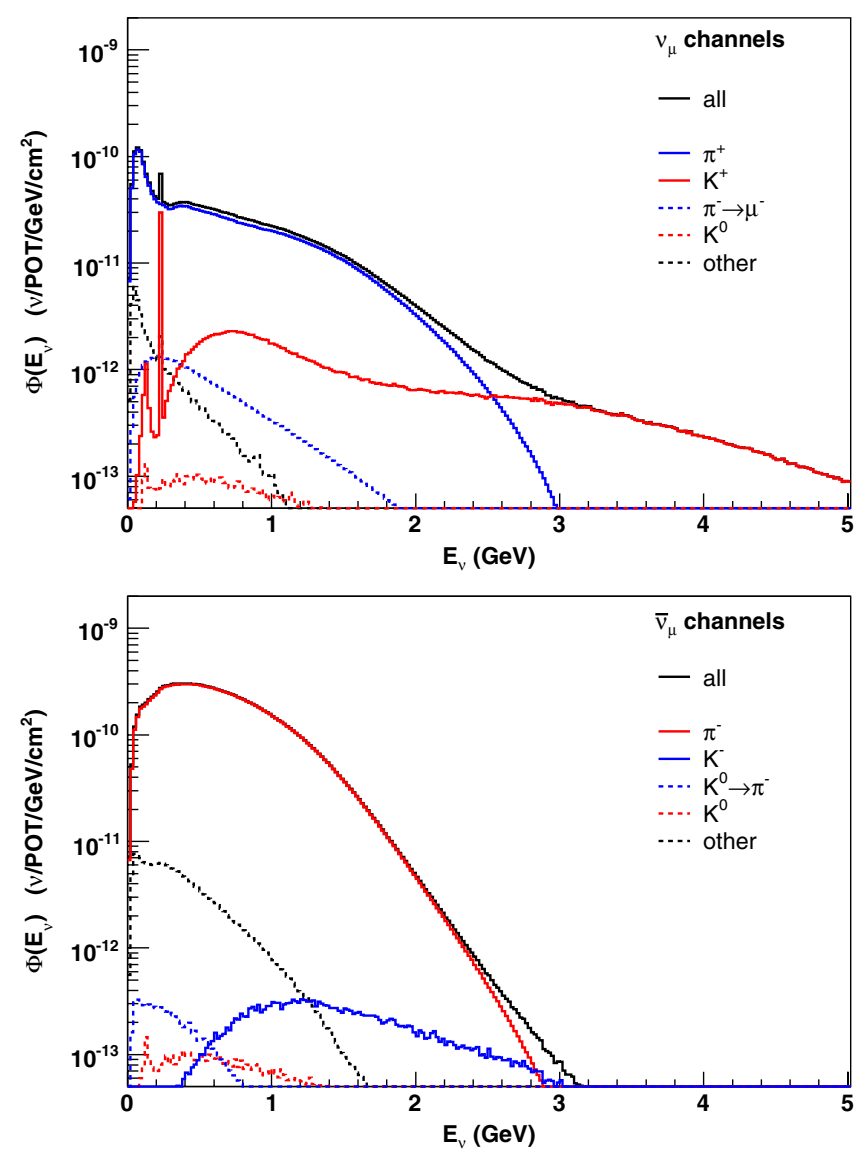

FIG. 31 (color online). Predicted $\nu_{\mu}$ (top) and $\bar{\nu}_{\mu}$ (bottom) fluxes at the MiniBooNE detector by parent meson species with horn in anti-neutrino mode. The black line is the total predicted flux, while all the subcomponents apart from the dashed black are from nucleon-induced meson production. The dashed black histogram includes all other contributions, primarily from meson decay chains initiated by meson-nucleus interactions.

energies are from two- and three-body $K^{+}$decays at rest. Because of the relative size of the $\pi^{+}$flux, however, they are not visible in the total $\nu_{\mu}$ flux. There is a small contribution to the flux from pions produced in the decay of kaons, and a similar contribution from tertiary mesoninduced production of other mesons that decay to produce $\nu_{\mu}$.

For the $\bar{\nu}_{\mu}$ in neutrino mode, $\pi^{-} \rightarrow \bar{\nu}_{\mu}$ flux is dominant at all energies. The next largest contribution comes from the $\pi^{+} \rightarrow \mu^{+} \rightarrow \bar{\nu}_{\mu}$ decay chain. For the $\nu_{\mu}$ flux, the analogous contribution from the $\pi^{-} \rightarrow \mu^{-} \rightarrow \nu_{\mu}$ decay chain is suppressed by the defocusing of the $\pi^{-}$. The kaon contribution is suppressed by the lower rate of $K^{-}$production relative to $K^{+}$production. Apart from low energies $(<200 \mathrm{MeV})$ the predicted $\bar{\nu}_{\mu}$ flux is typically $\sim 6 \%$ of the $\nu_{\mu}$ flux.

The channels through which $\nu_{e}$ and $\bar{\nu}_{e}$ are produced in neutrino mode are shown in Fig. 30. For the $\nu_{e}$ flux, the two dominant components are the $\pi^{+} \rightarrow \mu^{+} \rightarrow \nu_{e}$ decay
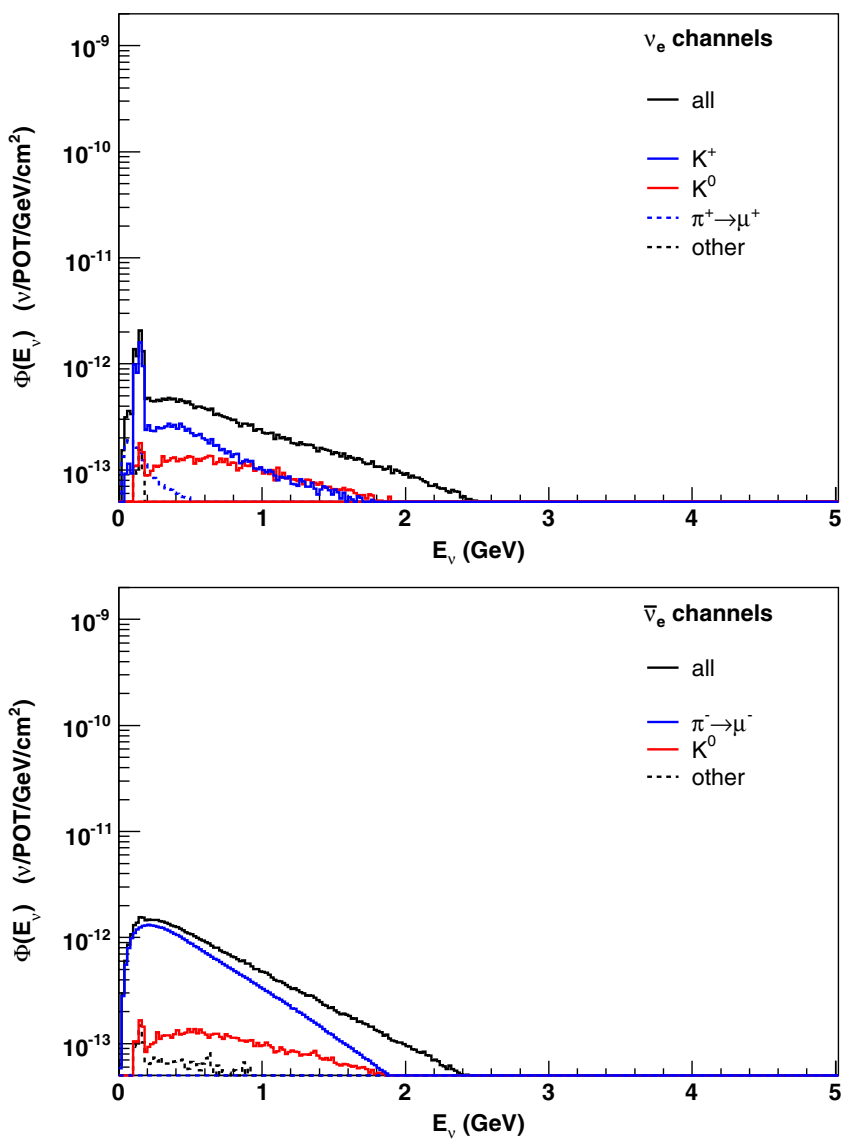

FIG. 32 (color online). Predicted $\nu_{e}$ (top) and $\bar{\nu}_{e}$ (bottom) fluxes at the MiniBooNE detector by parent meson species with horn in anti-neutrino mode. The black line is the total predicted flux, while all the subcomponents apart from the dashed black are from nucleon-induced meson production. The dashed black histogram includes all other contributions, primarily from meson decay chains initiated by meson-nucleus interactions.

chain and three-body $K^{+} \rightarrow \nu_{e}$ decay, where the former is dominant at low energies $(<1 \mathrm{GeV})$ and the latter is dominant at higher energies. The peak in the $K^{+} \rightarrow \nu_{e}$ spectrum at low energies is from the decay of $K^{+}$at rest (the peak from two-body decay is much smaller due to helicity suppression). For $\bar{\nu}_{e}$, the $\pi^{-} \rightarrow \mu^{-} \rightarrow \bar{\nu}_{e}$ flux contributes only at lower energies due to the defocusing of the $\pi^{-}$, and the $K^{-} \rightarrow \bar{\nu}_{e}$ contribution is suppressed both by the lower production rates and the defocusing. The rest of the spectrum is dominated by $K_{L}^{0}$ decay. As in the $\nu_{\mu} / \bar{\nu}_{\mu}$ case, the predicted $\bar{\nu}_{e}$ flux is $\sim 10 \%$ of the $\nu_{e}$ flux.

Figs. 31 and 32 show a similar composition for the predicted anti-neutrino mode flux. The $\bar{\nu}_{\mu}$ flux is dominated at all energies by $\pi^{-} \rightarrow \bar{\nu}_{\mu}$ decays; the suppressed production of $K^{-}$results in the contribution of $K^{-} \rightarrow \bar{\nu}_{\mu}$ being much smaller than the corresponding $K^{+} \rightarrow \nu_{\mu}$ contribution in neutrino mode. Furthermore, since the $K^{-}$that come to rest are captured, the $\bar{\nu}_{\mu}$ flux does not 
show the peaks from two-body and three-body decay-atrest at low energies that are found in the $\nu_{\mu}$ from $K^{+}$decay in both neutrino and anti-neutrino mode. It can also be seen that the high energy flux of $\nu_{\mu}$ is not substantially suppressed relative to the $\bar{\nu}_{\mu}$. In fact, despite the defocusing of $K^{+}$, the $K^{+} \rightarrow \nu_{\mu}$ flux is larger than that of the $K^{-} \rightarrow \bar{\nu}_{\mu}$ decay. This is due to the relative production rates and, at high energies, the leading particle effect where $\pi^{+}$and $K^{+}$ have a harder momentum spectrum relative to their negatively-charged counterparts. The high momentum of the particles that produce these neutrinos, along with their forward angular distribution, result in less defocusing from the horn for the wrong-sign component (positive (negative) particles for (anti-) neutrino mode). A similar effect is seen for the $\nu_{e} / \bar{\nu}_{e}$ components in anti-neutrino mode: while the $\bar{\nu}_{e}$ are dominated by $\mu^{-}$decays at energies below $2 \mathrm{GeV}$, the $K^{+} \rightarrow \nu_{e}$ flux is larger than the $K^{-} \rightarrow \bar{\nu}_{e}$ flux. A related observation is the fact that while the absolute rate of $\nu_{e} / \bar{\nu}_{e}$ from $K_{L}^{0}$ is unchanged from neutrino mode, the relative contribution is much stronger in anti-neutrino mode.

\section{SYSTEMATIC UNCERTAINTIES}

The systematic uncertainties in the neutrino flux prediction come from several sources:

(i) Proton delivery: The simulation determines the rate and spectrum of neutrinos per proton-on-target. This information is combined with the number of protons delivered to the target to determine the number of neutrinos passing through the MiniBooNE detector over the data collection period. As a result, the predicted number of neutrino interactions in the detector varies directly with the uncertainty in the number of protons-on-target. A related uncertainty arises from the optics of the proton beam which can change the expected number of protons interacting in the target (or elsewhere), changing the neutrino flux.

(ii) Particle Production: The uncertainties in the rate and spectrum of secondary particles produced in the $p$-Be interactions likewise affect the rate and spectrum of the neutrinos they produce. This is the dominant uncertainty.

(iii) Hadronic Interactions: The rate of hadronic interactions affect many aspects of the neutrino production, including the rate of $p$-Be interactions and the probability for mesons to survive possible hadronic interactions in the target or horn and decay to produce neutrinos. Uncertainties in the rate of these interactions affect both the rate and shape of the flux.

(iv) Horn magnetic field: The focusing properties of the horn change with the current as well as the distribution of the magnetic field within the conducting elements. Uncertainties in these properties result in spectral distortions of the neutrino flux. (v) Beam line geometry: Misalignments or displacements of the beam line components from their expected orientation and locations can affect the neutrino flux in many ways. For example, a misalignment can result in the detector being exposed to a different part of the neutrino flux than expected. A displacement of the target with respect to the horn can result in a variation in the focusing properties.

\section{A. Proton delivery}

The systematic uncertainties associated with the delivery of the primary proton beam to the beryllium target can be divided into two parts: the uncertainty in the number of protons delivered to the beam line and the uncertainty in the number which actually strike the target. Having entered the target, there are further uncertainties associated with how often the protons will interact to produce secondary particles based on the assumed hadronic cross sections; we consider these uncertainties in Sec. VII C.

As mentioned in Sec. II, the protons delivered to the BNB are measured by two toroids upstream of the target. The systematic uncertainty in the resulting spill-by-spill measurements has been estimated to be $2 \%$ based on uncertainties in the toroid circuit elements and uncertainties in the calibration procedure. Since the overall neutrino flux scales with the delivered protons, this source or error can be treated as an overall normalization uncertainty. The toroid measurements have been cross checked by measuring the activation on a gold foil inserted into the beam. The number of protons striking the foil inferred from this measurement agree with the toroid measurements within the $\sim 10 \%$ uncertainty of the measurement.

The effect of uncertainties in the primary beam optics, most notably the transverse profile and focusing and divergence properties, have been estimated by simulating the effects introduced by perturbing the default beam parameters. A number of different configurations, including varying the focal point across the length of the target, a "pin" configuration with no transverse spread or angular divergence, and a "pencil" configuration with transverse spread but no angular divergence, have been considered. The resulting changes to the number of protons expected to interact in the target is less than $1 \%$, which is taken as a systematic uncertainty in the overall normalization of the neutrino flux.

\section{B. Particle production}

The uncertainties in the particle production are summarized as a covariance matrix in the fitted parameters of the functions parametrizing the double differential cross section as described in Sec. V. The effect of these uncertainties is propagated to the neutrino flux by drawing random parameter vectors according to the covariance matrix via the Cholesky decomposition [21]. The resulting variation in the double differential meson production cross section at 

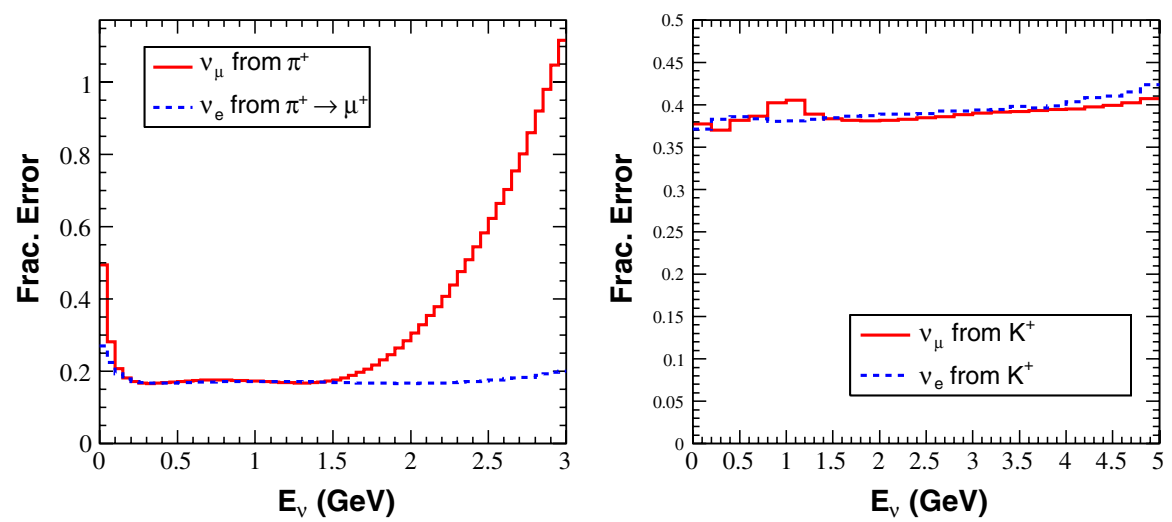

FIG. 33 (color online). Left: The fractional uncertainties in the $\pi^{+} \rightarrow \nu_{\mu}$ and $\pi^{+} \rightarrow \mu^{+} \rightarrow \nu_{e}$ flux with the horn in neutrino mode due to uncertainties in the $\pi^{+}$production in $p$-Be interactions. Right: Same for the $K^{+} \rightarrow \nu_{\mu}$ and $K^{+} \rightarrow \nu_{e}$ flux from uncertainties in the $K^{+}$production in $p$-Be interactions.

any point in $(p, \theta)$ can be evaluated with respect to the default value. The change in the neutrino flux can then be recalculated by assigning a weight corresponding to the ratio of the double differential cross section of the secondary particle producing the neutrino with the varied and default parameters.

In this way, the flux resulting from different production distributions summarized by alternate parameters can be calculated without rerunning the flux simulation. By accumulating the covariance of the flux distribution as the parameters are varied according to their covariance matrix, the uncertainties are propagated into the neutrino flux. This procedure is repeated for each parent particle species $\left(\pi^{+}, \pi^{-}, K^{+}, K_{L}^{0}\right)$, and for each neutrino species $\left(\nu_{\mu}, \nu_{e}, \bar{\nu}_{\mu}, \bar{\nu}_{e}\right)$ to obtain the total flux uncertainty, accounting for the correlated variations in the different neutrino species. This results in a covariance matrix for the predicted flux of each neutrino species from each of the meson species.
Figure 33 shows the fractional uncertainty in the neutrino flux from $\pi^{+}$and $K^{+}$production uncertainties, corresponding to the square-root of the diagonal entries of the covariance matrix resulting from the procedure described above divided by the predicted flux. In the left plot, the solid histogram shows the fractional uncertainty in the flux of neutrinos at the MiniBooNE detector from $\pi^{+} \rightarrow \nu_{\mu}$ produced in $p$-Be interactions due to the uncertainties in the $\pi^{+}$production. The strong correlation between the energy of the $\nu_{\mu}$ and the energy of the $\pi^{+}$which decayed to produce it results in a large rise in the fractional uncertainty at neutrino energies greater than $2 \mathrm{GeV}$ reflecting the large uncertainties in high-momentum pion production. Likewise, the uncertainty rises at low neutrino energies $(<200 \mathrm{MeV})$ due to the rise in the uncertainties for low momentum $\pi^{+}$production. Fortunately, relatively few neutrinos are produced in this region by the $\pi^{+}$decays; in the region below $1 \mathrm{GeV}$ where the $\pi^{+} \rightarrow \nu_{\mu}$ contribution is dominant, the uncertainty is approximately $17 \%$.
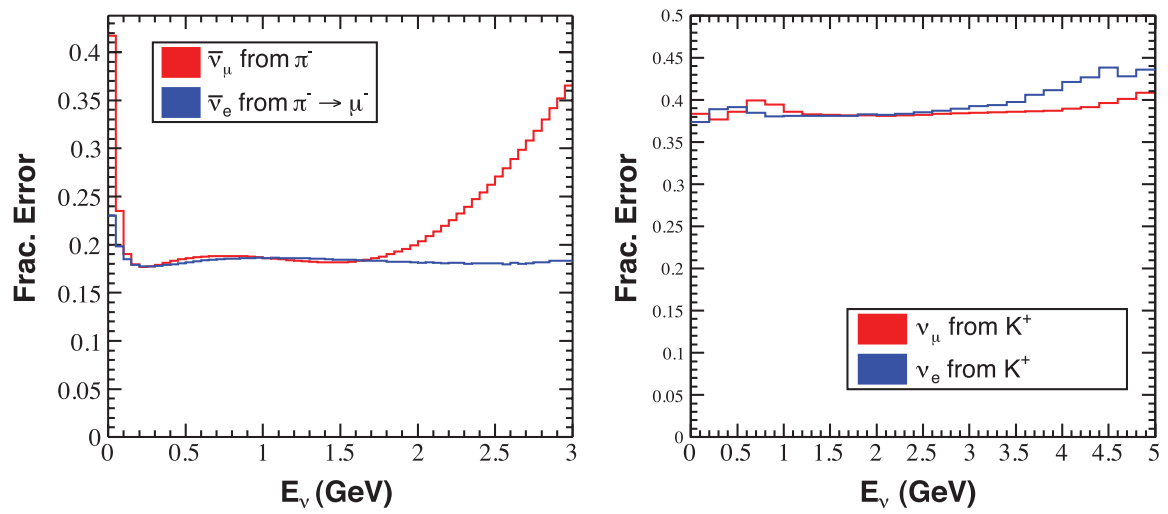

FIG. 34 (color). Left: The fractional uncertainties in the neutrino flux $\pi^{-} \rightarrow \bar{\nu}_{\mu}$ and $\pi^{-} \rightarrow \mu^{-} \rightarrow \bar{\nu}_{e}$ flux with the horn in antineutrino mode due to uncertainties in the $\pi^{-}$production in $p$-Be interactions. Right: Same for the $K^{+} \rightarrow \nu_{\mu}$ and $K^{+} \rightarrow \nu_{e}$ flux from uncertainties in the $K^{+}$production in $p$-Be interactions. 

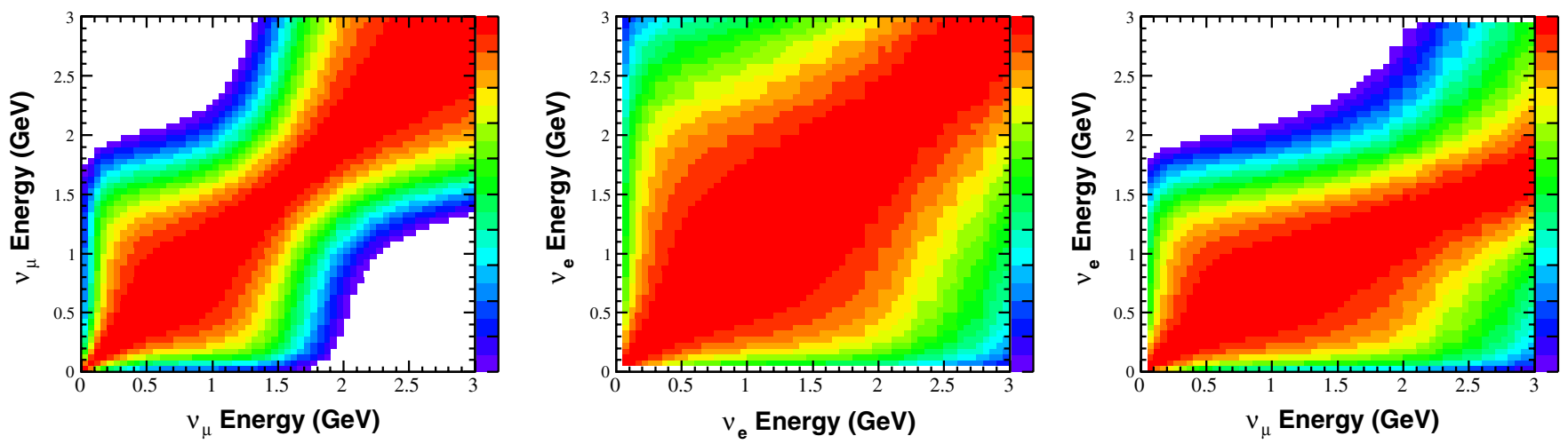

FIG. 35 (color online). Left: Correlation matrix for variations in the $\pi^{+} \rightarrow \nu_{\mu}$ flux due to uncertainties in $\pi^{+}$production in $p$-Be interactions with the horn in neutrino mode. Center/Right: Same for variations in the $\pi^{+} \rightarrow \mu^{+} \rightarrow \nu_{e}$ flux (center) and correlations between the $\pi^{+} \rightarrow \nu_{\mu}$ flux and the $\pi^{+} \rightarrow \mu^{+} \rightarrow \nu_{e}$ flux (right). The color scale on each plot ranges from 0 to 1 .

The dashed histogram shows the fractional uncertainty in the $\nu_{e}$ from the $\pi^{+} \rightarrow \mu^{+} \rightarrow \nu$ decay chain resulting from the uncertainties in the $\pi^{+}$production, the primary channel for low energy $(<1 \mathrm{GeV}) \nu_{e}$ flux. Since the correlation between the energy of the $\nu_{e}$ and the $\pi^{+}$which produces it is weak due to the three-body decay of the muon, the uncertainties are more uniform as a function of energy.

The right plot in Fig. 33 likewise shows the fractional uncertainty for the flux of $\nu_{\mu}$ and $\nu_{e}$ resulting from the decay of $K^{+}$produced in $p$-Be interactions due to the uncertainties in the $K^{+}$production. These channels are the primary contribution for $\nu_{\mu}$ with energy greater than $2.3 \mathrm{GeV}$ and $\nu_{e}$ with energy greater than $1.2 \mathrm{GeV}$. Because of the larger $K^{+}$mass, the correlation between the momentum of the $\mathrm{K}^{+}$and the neutrinos from its decay is also weak.

Figure 34 shows the corresponding plots for the horn in anti-neutrino mode, where the $\pi^{-} \rightarrow \bar{\nu}_{\mu}$ flux is dominant. While the corresponding charged kaon channel would be $K^{-} \rightarrow\left(\bar{\nu}_{\mu} / \bar{\nu}_{e}\right)$, the $K^{+} \rightarrow\left(\nu_{\mu} / \nu_{e}\right)$ uncertainties are shown instead, since the contribution of this channel is larger.

Figure 35 shows the bin-to-bin correlations in the uncertainties related to the pion production. The left and center plots show the correlation matrix associated with the fractional uncertainties in the $\pi^{+} \rightarrow \nu_{\mu}$ and $\pi^{+} \rightarrow$ $\mu^{+} \rightarrow \nu_{e}$ flux in $p$-Be interactions, respectively. The $\pi^{+} \rightarrow \nu_{\mu}$ flux exhibit correlations that are strongest between nearby bins, with the correlations steadily weakening for bins separated by more than several hundred $\mathrm{MeV}$. The $\pi^{+} \rightarrow \mu^{+} \rightarrow \nu_{e}$ flux, however, shows correlation between energies which are more widely separated, as would be expected from the three-body decay of the $\mu^{+}$that produces this flux. The right plot in Fig. 35 shows the correlations between the uncertainties in the two components of the $\pi^{+}$flux. As expected, the $\pi^{+} \rightarrow \nu_{\mu}$ flux at a given energy is most strongly correlated with $\pi^{+} \rightarrow$ $\mu^{+} \rightarrow \nu_{e}$ flux at lower energies.

Figure 36 shows similar correlations for the $K^{+} \rightarrow \nu_{\mu}$ (left) and $K^{+} \rightarrow \nu_{e}$ (center) fluxes. The situation is quite different from the $\pi^{+}$flux; the uncertainties are correlated
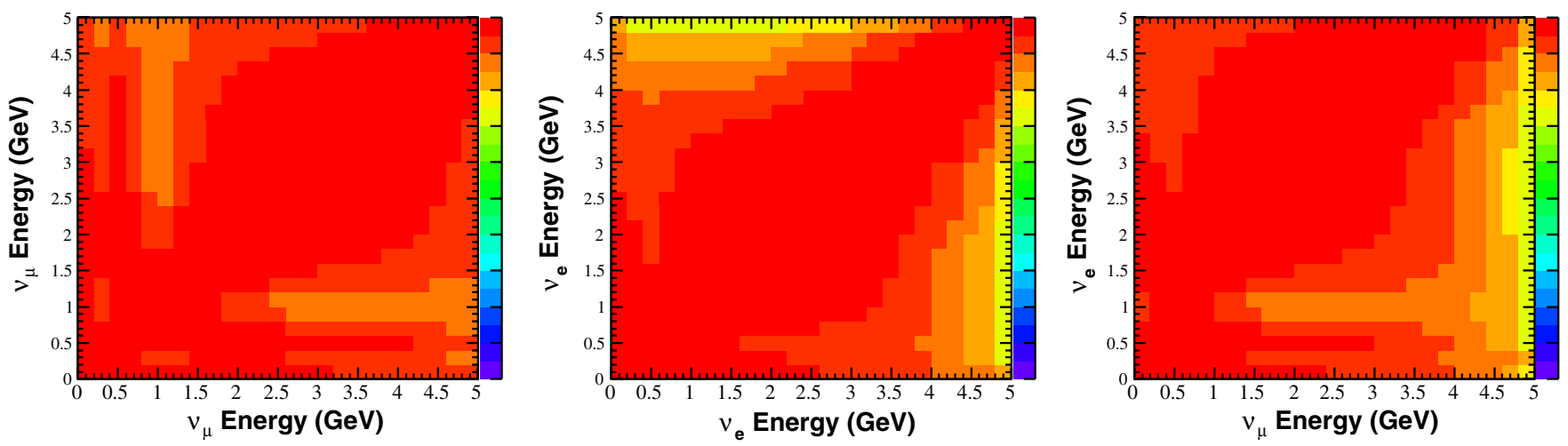

FIG. 36 (color online). Left: Correlation matrix for variations in the $K^{+} \rightarrow \nu_{\mu}$ flux due to uncertainties in $K^{+}$production in $p$-Be interactions with the horn in neutrino mode. Center/Right: Same for variations in the $K^{+} \rightarrow \nu_{e}$ flux (center) and correlations between the $K^{+} \rightarrow \nu_{\mu}$ flux and the $K^{+} \rightarrow \nu_{e}$ flux (right). The color scale on each plot ranges from 0 to 1 . 

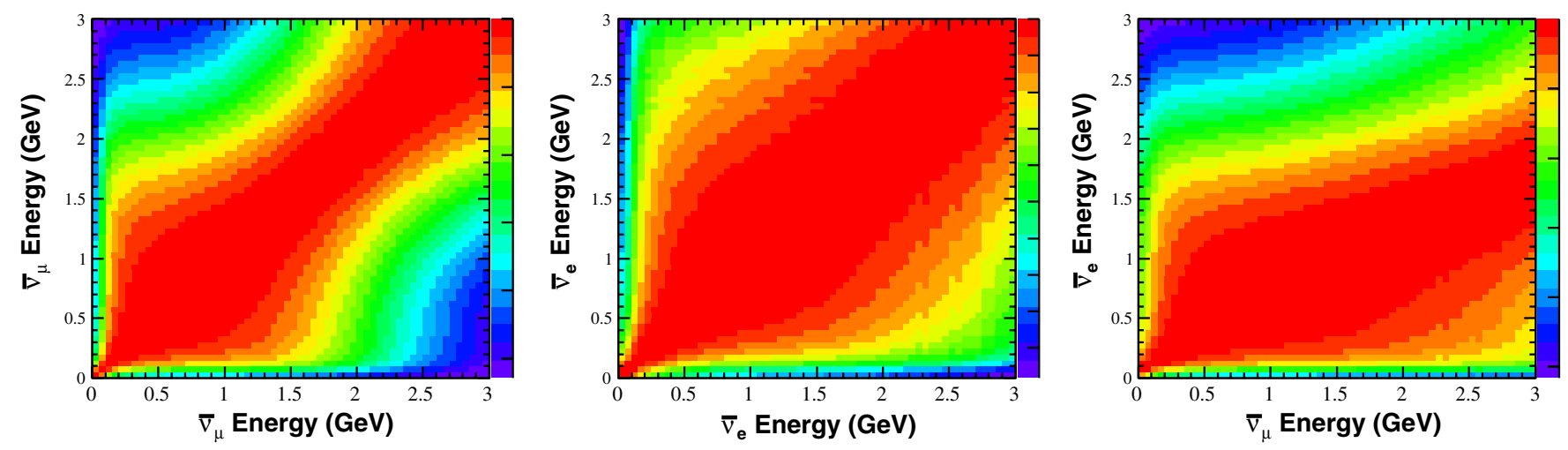

FIG. 37 (color online). Left: Correlation matrix for variations in the $\pi^{-} \rightarrow \bar{\nu}_{\mu}$ flux due to uncertainties in $\pi^{-}$production in $p$-Be interactions with the horn in anti-neutrino mode. Center/Right: Same for variations in the $\pi^{-} \rightarrow \mu^{-} \rightarrow \bar{\nu}_{e}$ flux (center) and correlations between the $\pi^{-} \rightarrow \bar{\nu}_{\mu}$ flux and the $\pi^{-} \rightarrow \mu^{-} \rightarrow \bar{\nu}_{e}$ flux (right). The color scale on each plot ranges from 0 to 1 .
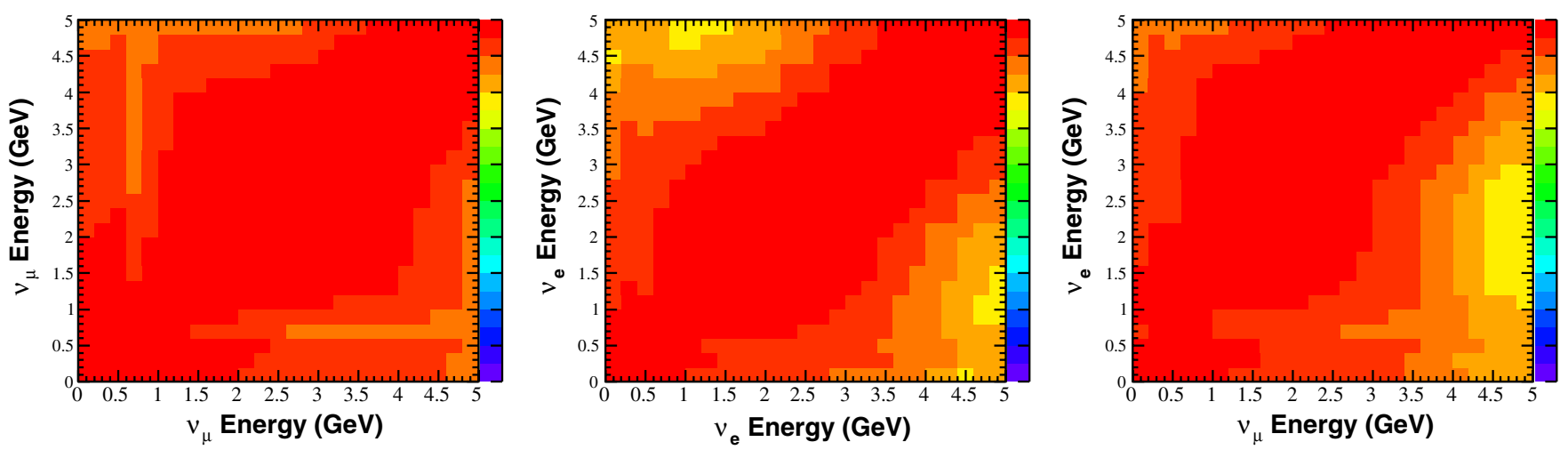

FIG. 38 (color online). Left: Correlation matrix for variations in the $K^{+} \rightarrow \nu_{\mu}$ flux due to uncertainties in $K^{+}$production in $p$-Be interactions with the horn in anti-neutrino mode. Center/Right: Same for variations in the $K^{+} \rightarrow \nu_{e}$ flux (center) and correlations between the $K^{+} \rightarrow \nu_{\mu}$ flux and the $K^{+} \rightarrow \nu_{e}$ flux (right). The color scale on each plot ranges from 0 to 1 .

across energies for each flux. Likewise, the right plot, which shows the correlations between the uncertainties in the two sources of neutrinos, is also strongly correlated. This reflects the large normalization uncertainty assigned to the $K^{+}$production; the variations in the $K^{+}$production correspond mainly to shifts in the overall rate.

Figs. 37 and 38 show the corresponding plots for the horn in anti-neutrino mode. Once again, the correlations for the $K^{+} \rightarrow\left(\nu_{\mu} / \nu_{e}\right)$ uncertainties are shown instead of the corresponding $K^{-} \rightarrow\left(\bar{\nu}_{\mu} / \bar{\nu}_{e}\right)$. The results show a similar pattern of correlations to that observed in the neutrino mode.

Because of the large uncertainties in the flux prediction from particle production, which result not from uncertainties in the measured particle production but from the parametrizations used to describe the measurements, an alternative description of the measurements was investigated. In this method, the particle production data are interpolated as splines using the DCSPLN routine from CERNLIB [13]. The two-dimensional measurements of the double differential cross section in $p$ and $\theta$ are inter- polated as splines first in $\theta$ at fixed values of $p$. The resulting splines in $\theta$ are then interpolated to produce values as a function of $p$. The splines also extrapolate the double differential cross section into region where measurements do not exist, most notably at low pion momentum $(<700 \mathrm{MeV} / c)$.

Uncertainties in the particle production are derived by varying the measured double differential cross sections according to the $78 \times 78$ covariance matrix describing the uncertainties in the measurements. Each variation results in an alternate set of double differential cross sections that is interpolated. By repeating this process (in practice 1000 times) a covariance matrix describing the pion production variations can be derived that can be used to propagate the uncertainties into the predicted neutrino flux. As a result of this procedure, the pion production uncertainties can be derived in a manner that is directly connected with the experimental uncertainties, circumventing the difficulties associated with parametrizing the double differential cross section and reducing the uncertainties in the neutrino flux significantly. In the core of the 


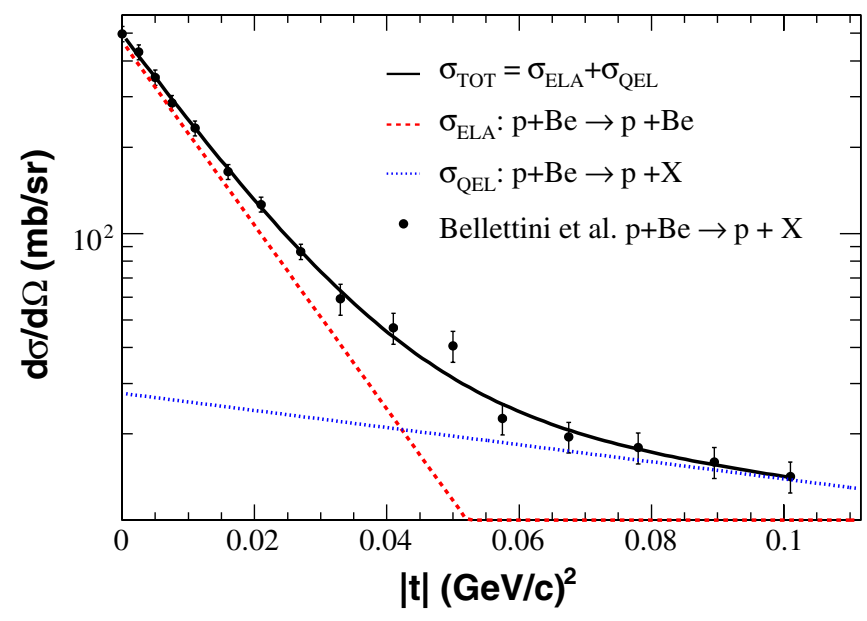

FIG. 39 (color online). Observed $q^{2}$ distribution from Bellettini et al. [59] for $20 \mathrm{GeV} / c$ p-Be scattering. The solid line represents the fit to two exponential distributions with the dashed and dotted lines showing the elastic and quasielastic contributions, respectively.

$\nu_{\mu}$ energy distribution $(0.5-1.0 \mathrm{GeV})$ the $\nu_{\mu}$ flux uncertainty resulting from this method is 5-7\%. At low energies $(<500 \mathrm{MeV})$, where measurements do not exist, the uncertainties rise to $\sim 20 \%$. At higher energies $>1 \mathrm{GeV}$, where the measurements have larger uncertainties, the $\nu_{\mu}$ flux uncertainty rises to $\sim 10 \%$. This method was not used for the neutrino oscillation results reported in Ref. [2] but illustrates how the additional uncertainties associated with the parametrization of particle production measurements can be avoided in future analyses.

\section{Hadronic interactions}

Uncertainties due to hadronic interactions are considered by varying the components of the hadronic cross sections. First, the total hadronic cross section $\sigma_{\mathrm{TOT}}$, the total inelastic $\sigma_{\mathrm{INE}}$ and the quasielastic $\sigma_{\mathrm{QEL}}$ cross sections are separately varied for nucleons on beryllium and aluminum. Second, the same is done for the pion cross sections. In each case, the variations are a flat, momentumindependent offset. Because of the various relations between the cross sections, the variation of $\sigma_{\mathrm{TOT}}$ results in a variation of $\sigma_{\mathrm{ELA}}\left(\sigma_{\mathrm{INE}}\right.$ is fixed). When $\sigma_{\mathrm{INE}}$ is varied, the balance between $\sigma_{\mathrm{ELA}}$ and $\sigma_{\mathrm{INE}}$ is changed, while keeping their total at $\sigma_{\mathrm{TOT}}$. Finally, when $\sigma_{\mathrm{QEL}}$ is varied, the relative proportion of $\sigma_{\mathrm{QEL}}$ to the cross section for all other inelastic processes is changed, while keeping their sum $\left(\sigma_{\mathrm{INE}}\right)$ fixed.

The variations for $\sigma_{\mathrm{TOT}}$ are based on the agreement of the Glauber model calculations with the available $n$-nucleus measurements, shown in Fig. 11. The deviations of the measurements from the model are used to set the magnitude of the variation. These variations are also applied to $\sigma_{\mathrm{TOT}}$ for $\pi^{ \pm}$-nucleus measurements by scaling the
TABLE XIII. Cross section variations for systematic studies. For each hadron-nucleus cross section type, the momentumdependent cross section is offset by the amount shown.

\begin{tabular}{lcccccc}
\hline \hline & \multicolumn{2}{c}{$\Delta \sigma_{\mathrm{TOT}}(\mathrm{mb})$} & \multicolumn{2}{c}{$\Delta \sigma_{\mathrm{INE}}(\mathrm{mb})$} & \multicolumn{2}{c}{$\Delta \sigma_{\mathrm{QEL}}(\mathrm{mb})$} \\
& $\mathrm{Be}$ & $\mathrm{Al}$ & $\mathrm{Be}$ & $\mathrm{Al}$ & $\mathrm{Be}$ & $\mathrm{Al}$ \\
\hline$(p / n)-(\mathrm{Be} / \mathrm{Al})$ & \pm 15.0 & \pm 25.0 & \pm 5 & \pm 10 & \pm 20 & \pm 45 \\
$\pi^{ \pm}-(\mathrm{Be} / \mathrm{Al})$ & \pm 11.9 & \pm 28.7 & \pm 10 & \pm 20 & \pm 11.2 & \pm 25.9 \\
\hline \hline
\end{tabular}

variations from the nuclear case by the ratio of $\sigma_{\mathrm{TOT}}$ at high momentum in the two cases. Since there are no $\pi^{ \pm}$-nucleus $\sigma_{\text {TOT }}$ data above the $\Delta(1232)$ resonance to verify the model, this assumes that the model works as well for $\pi^{ \pm}$-nucleus interactions as it does for nucleonnucleus interactions.

The variations for $\sigma_{\text {INE }}$ are similarly set by the deviations of the measurements from the parametrization. Here the deviations are smaller since the parametrizations are derived directly from the data; the variations are intended to incorporate the uncertainties in the measurements.

The uncertainties for $\sigma_{\mathrm{QEL}}$ are set by comparing the calculated cross section to the inferred $\sigma_{\mathrm{QEL}}$ from the measured $q^{2}$ distribution in $p$-Be scattering from Ref. [59] shown in Fig. 39. This distribution is fit to the sum of two exponentials corresponding to the elastic and quasielastic scattering components. The fitted slope of the quasielastic component is consistent with free nucleonnucleon elastic scattering and leads a ratio $\sigma_{\mathrm{QEL}} / \sigma_{\mathrm{ELA}}$ of 0.6. Since the free nucleon cross sections and $\sigma_{\mathrm{TOT}}$ do not change appreciably from $8.89 \mathrm{GeV} / c$ to $20 \mathrm{GeV} / c$, the value and uncertainty of $\sigma_{\mathrm{ELA}}$ at $8.89 \mathrm{GeV} / c$ can be used to obtain $\sigma_{\mathrm{QEL}}=44 \pm 9 \mathrm{mb}$ at the same beam momentum. This can be compared to the $34.9 \mathrm{mb}$ obtained from the shadowed scattering model. An uncertainty of $20 \mathrm{mb}$ is assigned to $\sigma_{\mathrm{QEL}}$ to account for both the difference and the uncertainty from the Bellettini measurement. The uncertainty for nucleon-aluminum scattering is obtained by scaling this uncertainty by the ratio of the predicted $\sigma_{\mathrm{QEL}}$ in aluminum and beryllium. The variation for $\sigma_{\mathrm{QEL}}$ in $\pi^{ \pm}$-nucleus scattering is obtained by scaling the variation in nucleon-nucleus scattering by the ratio of $\sigma_{\mathrm{QEL}}$ for $\pi^{ \pm}$ and nucleons.

Table XIII summarizes the variations in all six hadronnucleus combinations. Of these variations, the variations in $\sigma_{\mathrm{QEL}}$ have the largest effect.

\section{Horn magnetic field modeling}

Uncertainties on two properties of the horn magnetic field result in systematic uncertainties in the neutrino flux. The first is the horn current. The commercial current transformers (Stangenes Industries 3-0.002) have a rated accuracy of $0.5 \%$. The effect of a $1 \mathrm{kA}$ variation in the nominal horn current $(174 \pm 1 \mathrm{kA})$ is simulated to set the systematic uncertainty. 
A second source of uncertainty arises from the modeling of the current within the inner cylinder due to the so-called "skin effect". The skin effect allows for the magnetic field to penetrate into the conductor, increasing the effective magnetic field experienced by particles traversing through the inner conductor into the horn cavity. This is important for particles produced at small angles (particularly high momentum) that barely penetrate into the horn cavity before exiting the front of the horn into the collimator region. For these particles, the bulk of the magnetic field seen by the particle in this trajectory may come from the field within the inner conductor.

The expected current distribution in a cylindrically symmetric configuration was numerically evaluated and found to be well-approximated by a current density exponentially decreasing from the outer surface of the inner conductor with a decay length set by the skin depth $(1.4 \mathrm{~mm})$. The magnetic field configuration corresponding to this current density is simulated and taken as the default configuration. The simulation is also run without the skin effect, simulating the situation where the current density lies entirely on the outer surface of the inner conductor of the horn, resulting in no magnetic field penetration. The difference between these two configurations, a few percent in the predicted neutrino flux for $\nu_{\mu}$ with energies $<1 \mathrm{GeV}$ and up to $18 \%$ for $\nu_{\mu}$ with energies $\sim 2 \mathrm{GeV}$, is considered a systematic uncertainty.

TABLE XIV. Variations in the total flux of each neutrino species in neutrino mode due to the systematic uncertainties.

\begin{tabular}{lrrrr}
\hline \hline Source of Uncertainty & \multicolumn{1}{c}{$\nu_{\mu}$} & \multicolumn{1}{c}{$\bar{\nu}_{\mu}$} & \multicolumn{1}{c}{$\nu_{e}$} & \multicolumn{1}{c}{$\bar{\nu}_{e}$} \\
\hline Proton delivery & $2.0 \%$ & $2.0 \%$ & $2.0 \%$ & $2.0 \%$ \\
Proton optics & $1.0 \%$ & $1.0 \%$ & $1.0 \%$ & $1.0 \%$ \\
$\pi^{+}$production & $14.7 \%$ & $1.0 \%$ & $9.3 \%$ & $0.9 \%$ \\
$\pi^{-}$production & $0.0 \%$ & $16.5 \%$ & $0.0 \%$ & $3.5 \%$ \\
$K^{+}$production & $0.9 \%$ & $0.2 \%$ & $11.5 \%$ & $0.3 \%$ \\
$K^{0}$ production & $0.0 \%$ & $0.2 \%$ & $2.1 \%$ & $17.6 \%$ \\
Horn field & $2.2 \%$ & $3.3 \%$ & $0.6 \%$ & $0.8 \%$ \\
Nucleon cross sections & $2.8 \%$ & $5.7 \%$ & $3.3 \%$ & $5.6 \%$ \\
Pion cross sections & $1.2 \%$ & $1.2 \%$ & $0.8 \%$ & $0.7 \%$ \\
\hline \hline
\end{tabular}

TABLE XV. Variations in the total flux of each neutrino species in anti-neutrino mode due to the systematic uncertainties.

\begin{tabular}{lrrrr}
\hline \hline Source of Uncertainty & \multicolumn{1}{c}{$\nu_{\mu}$} & \multicolumn{1}{c}{$\bar{\nu}_{\mu}$} & \multicolumn{1}{c}{$\nu_{e}$} & \multicolumn{1}{c}{$\bar{\nu}_{e}$} \\
\hline Proton delivery & $2.0 \%$ & $2.0 \%$ & $2.0 \%$ & $2.0 \%$ \\
Proton optics & $1.0 \%$ & $1.0 \%$ & $1.0 \%$ & $1.0 \%$ \\
$\pi^{+}$production & $13.8 \%$ & $0.1 \%$ & $2.1 \%$ & $0.1 \%$ \\
$\pi^{-}$production & $0.5 \%$ & $17.5 \%$ & $0.0 \%$ & $13.6 \%$ \\
$K^{+}$production & $3.1 \%$ & $0.0 \%$ & $22.3 \%$ & $0.4 \%$ \\
$K^{0}$ production & $0.1 \%$ & $0.0 \%$ & $6.1 \%$ & $3.9 \%$ \\
Horn field & $1.5 \%$ & $1.0 \%$ & $3.2 \%$ & $1.5 \%$ \\
Nucleon cross sections & $6.2 \%$ & $2.1 \%$ & $6.2 \%$ & $2.5 \%$ \\
Pion cross sections & $1.5 \%$ & $1.2 \%$ & $1.6 \%$ & $1.5 \%$ \\
\hline \hline
\end{tabular}

\section{E. Geometry uncertainties}

Variations in the geometric configuration of the beam line are simulated to investigate their effect on the neutrino flux. These variations include moving the target position relative to the rest of the beam line (in particular the horn), varying the radius of the decay pipe, and moving the collimator along the beam axis and changing its aperture. The magnitudes of the geometric perturbations which are required to effect a substantial $(>1 \%)$ change in the flux are well outside of what are considered the tolerances and precision of the constructed beam line. As a result, no significant systematic uncertainty is assigned to the beam line geometry.

\section{F. Summary of systematic uncertainties}

Tables XIV and XV summarize the variations in the total flux for each neutrino species resulting from the systematic uncertainties discussed in this section. By far the largest uncertainty arises from the particle production uncertainties. Much of this uncertainty arises not from the accuracy of the measurements, but from the parametrizations used to summarize the double differential cross sections. These latter contributions manifest as inflated uncertainties on the parameters resulting from the $\chi^{2} / \mathrm{DOF}$ at the best-fit values and other considerations such as the dependence on the choice of parametrization.

The flux-averaged uncertainties provide a rough gauge to the relative size of the various uncertainties; they are not used in the $\nu_{\mu} \rightarrow \nu_{e}$ oscillation analysis. As seen in Sec. VII B, the uncertainties can vary significantly with energy and exhibit correlations across energies and neutrino species. In the $\nu_{\mu} \rightarrow \nu_{e}$ oscillation analysis, uncertainties are propagated with covariance matrices where the energy-dependent variations in uncertainties and correlations are taken into account.

The top two plots of Fig. 40 illustrate the effects from the largest sources of systematic uncertainty, apart from the particle production uncertainties which have already been discussed, on the predicted $\pi^{+} \rightarrow \nu_{\mu}$ flux at the MiniBooNE detector with the horn in neutrino mode. The largest effect comes from the presence or absence of the skin effect in the conduction of the horn current along the inner conductor of the horn. The effect is particularly large for high energy neutrinos $(>1 \mathrm{GeV})$ due to the correlation of the pion momentum with angle (higher momentum $\pi^{+}$tend to be produced in the forward direction). As mentioned in Sec. VIID, these particles will usually have the largest change in the amount of magnetic field experienced in traversing from the target, through the horn, and into the decay region. However, for very high momentum $\pi^{+}(>4 \mathrm{GeV} / c)$, the production is collimated to such an extent that an increasing part of the production never enters the horn, and instead travels alongside the target into the decay region without traversing the inner conductor. For these $\pi^{+}$the skin effect is 

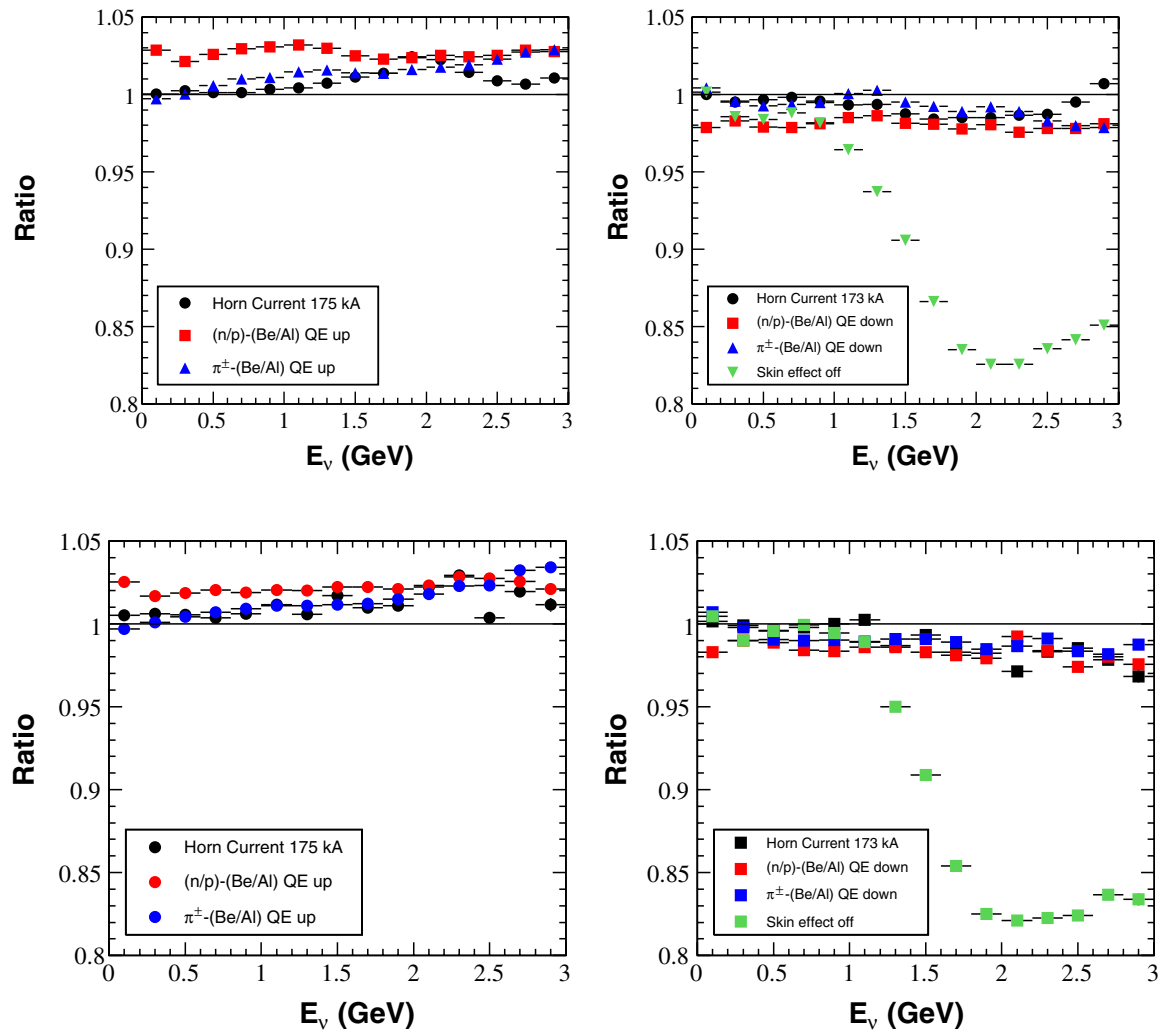

FIG. 40 (color online). Top: Change in the $\nu_{\mu}$ flux from $\pi^{+}$due to the dominant sources of systematic uncertainty apart from particle production. Left: Ratio of flux from increased horn current (circles), increased nucleon-nucleus quasielastic cross section (squares) and increased pion-nucleus quasielastic cross section (upward triangles) to the default flux. Right: Ratio of flux from decreased horn current (circles), decreased nucleon-nucleus quasielastic cross section (squares), decreased pion-nucleus quasielastic cross section (upward triangles), and turning off the skin effect (downward trianges), to the default flux. Bottom: Same for predicted $\bar{\nu}_{\mu}$ flux from $\pi^{-}$in antineutrino mode.

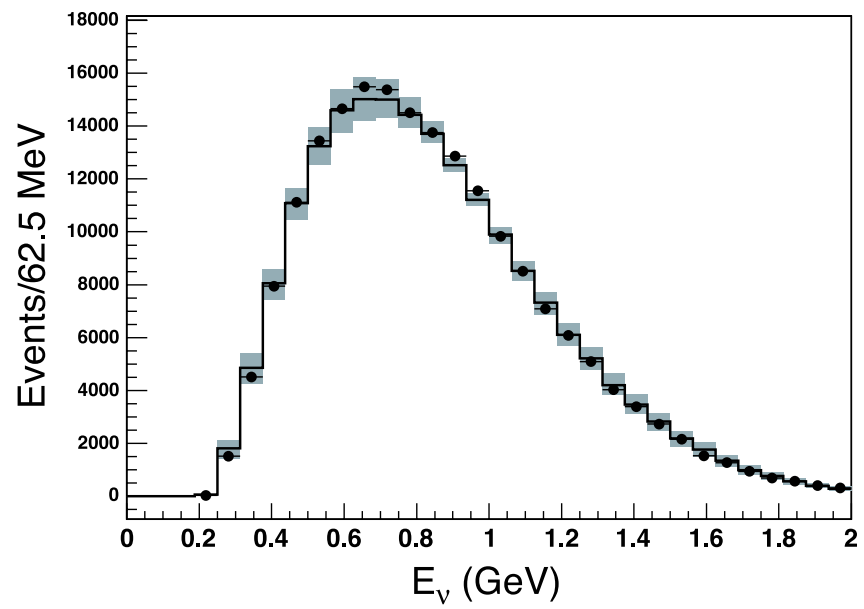

FIG. 41 (color online). Comparison of the observed (points) and predicted (histogram) energy distribution in $\nu_{\mu} \mathrm{CCQE}$ events selected in the MiniBooNE data. A normalization factor of 1.21 has been applied to the predicted distribution as described in the text. The error bars on the predicted distribution are the estimated uncertainties in the shape of the spectrum once the normalization has been fixed to match the data. irrelevant; as a result, the effect diminishes for the high energy neutrinos associated with the decay of these pions.

The next largest source of systematic uncertainty is from the magnitude of the hadron-nucleus quasielastic cross section. This effect is investigated for nucleon-nucleus and pion-nucleus cross sections separately. As discussed in Sec. VII C, in the nucleon-nucleus case, larger quasielastic cross section results in more protons emerging from the inelastic interactions with energies close to the primary energies. The interactions of these secondary protons is much like that of the primary protons. As a result, there is an overall increase in the particle production and the neutrino flux. For pions, an increase in the quasielastic cross section increases the effective hadronic transparency of the material which intervenes between the production of the pion and its decay (primarily the target and the horn). The effect is largest for forward particles (which tend to be at higher momentum) which traverse more material. As a result, an increase in the pion quasielastic cross section increases the neutrino flux with an energy dependence that favors high energy neutrinos. These trends for nucleon and 
pion quasielastic cross section variations are evident in Fig. 40. The bottom plot of Fig. 40 shows the same summary for the predicted $\pi^{-} \rightarrow \bar{\nu}_{\mu}$ flux at MiniBooNE with the horn in anti-neutrino mode. The pattern of systematic uncertainties is similar to that observed for the $\pi^{+} \rightarrow \nu_{\mu}$ flux in neutrino mode.

\section{COMPARISON TO OBSERVED NEUTRINO EVENTS}

The observed energy spectrum of $\nu_{\mu}$ charged-current quasielastic (CCQE) events at MiniBooNE in $5.6 \times 10^{20}$ protons-on-target taken in neutrino-enhanced mode is compared to the predicted spectrum in Fig. 41. The sample of $\nu_{\mu}$ CCQE interactions is obtained by selecting events consistent with a single muon-like Cherenkov ring in the detector, and with activity consistent with muon decay-atrest following the primary neutrino interaction. The predicted neutrino energy spectrum results from generating simulated neutrino interactions with the NUANCE neutrino event generator [60] according to the predicted neutrino fluxes and propagating the predicted final state particles through a detailed Monte Carlo simulation of the MiniBooNE detector [61]. The neutrino energy is calculated using the reconstructed muon energy and angle relative to the beam axis, assuming that the event is CCQE $\left(\nu_{\mu}+n \rightarrow \mu^{-}+p\right)$. Identical selection criteria are applied to the data. Details of the event selection and tuning of the NUANCE event generator can be found in Ref. [3]. Systematic uncertainties in the shape of the spectrum from the neutrino flux prediction, cross section model, and detector model are represented by the grey squares. The predicted purity of the selected events in $\nu_{\mu} \mathrm{CCQE}$ interactions is $74 \%$ with charged-current single pion production channels as the dominant source of background.

The observed rate of interactions is factor of $1.21 \pm 0.24$ higher than the rate predicted by the nominal simulation; the predicted spectrum in Fig. 41 has been scaled by this factor to facilitate the comparison of the spectrum shapes. While this is a sizable discrepancy, the uncertainties resulting from the predicted flux, the neutrino cross section model and the detector simulation are such that the scale factor is compatible with unity as indicated by its uncertainty. We note that the neutrino cross section parameters measured in Ref. [3] are derived from the $Q^{2}$ distribution and not the rate or energy spectrum of the observed events and that the neutrino flux prediction has not be adjusted in any way in response to the observed neutrino data apart from this normalization factor.

\section{CONCLUSIONS}

The neutrino flux at MiniBooNE is predicted by a detailed Geant4-based neutrino flux simulation. The Geant4 framework allows for a realistic representation of the beam line geometry and accounting of the electromagnetic and hadronic effects experienced by particles as they traverse the beam line. The software framework incorporates a number of custom features that have been tailored to the needs of the analyses at MiniBooNE. In particular, the properties of key hadronic processes, most notably the cross sections of nucleons and pions on beryllium and aluminum, and the particle production properties of $p-\mathrm{Be}$ interactions, have been tuned based on external measurements wherever possible. These have been summarized in a number of parametrizations that describe the momentum dependence of the overall cross sections, as well as the multiplicity and kinematic properties of the relevant secondary particle species in the primary $p$-Be interactions. The simulation also accounts for the measured properties of the primary proton beam.

A separate model controls the kinematics of neutrinos resulting from the decays of mesons propagated to their point of decay in the Geant 4 simulation. This model accounts for polarization effects as well as nontrivial decay form factors, and reflects the latest knowledge of key kaon branching fractions. The geometric acceptance of the neutrinos at the MiniBooNE detector is also handled. Both software frameworks employ a number of statistical enhancement techniques that reduce the uncertainties overall and enhance the statistical precision in kinematic regions and channels where important contributions to the flux may come from processes that are small in relation to the dominant channels.

The flexibility of the framework allows the determination of a number of systematic uncertainties by varying parameters within the simulation. In this way, the effect of varying hadronic cross sections and different horn currents can be estimated. By recording the kinematics of the secondary mesons at production, the uncertainties in the production differential cross sections can be propagated through reweighting without rerunning the simulation. The study of systematic uncertainties indicate that the dominant uncertainty arises from the particle production. These uncertainties arise not only from the intrinsic uncertainties in the particle production measurements, but also from the parametrizations used to model the differential cross sections. The resulting neutrino flux predictions and uncertainties are a critical element of the Monte Carlo simulation chain at MiniBooNE, where they are combined with the NUANCE neutrino event generator [60] and a detector Monte Carlo simulation [61] to determine the rate and properties of neutrino interactions in the detector. Comparison of the predicted event rate and spectrum with a sample of $\nu_{\mu}$ charged-current quasielastic events observed at MiniBooNE indicate that the spectrum is reproduced well by the simulation while there is a sizable discrepancy in the overall rate, with the observed data rate a factor $1.21 \pm 0.24$ higher than predicted. Because of the large uncertainty in the predicted rates, the observed and predicted rates are compatible. 


\section{ACKNOWLEDGMENTS}

The MiniBooNE collaboration acknowledges support from the Department of Energy and the National Science Foundation of the United States. We are grateful to Fermilab for hosting the experiment and thank the Accelerator Division for the excellent accelerator performance. We thank Los Alamos National Laboratory for
LDRD funding. We acknowledge Bartoszek Engineering for the design of the focusing horn. This research was done using resources provided by the Open Science Grid, which is supported by the NSF and DOE-SC. We also acknowledge the use of the LANL PINK cluster and CONDOR software in the analysis of the data.
[1] A. Aguilar et al. (LSND), Phys. Rev. D 64, 112007 (2001).

[2] A. A. Aguilar-Arevalo et al. (MiniBooNE), Phys. Rev. Lett. 98, 231801 (2007).

[3] A. A. Aguilar-Arevalo et al. (MiniBooNE), Phys. Rev. Lett. 100, 032301 (2008).

[4] J. R. Monroe, Report No. FERMILAB-THESIS-2006-44, 2006.

[5] R. B. Patterson, Report No. FERMILAB-THESIS-200719.

[6] $8 \mathrm{GeV}$ Beam Technical Design Report, http://www-boone. fnal.gov/publicpages/8gevtdr_2.0.ps.gz.

[7] T. R. Kobilarcik (MiniBooNE), Presented at 5th International Workshop on Neutrino Beams and Instrumentation (NBI 2005), Batavia, Illinois, 2005.

[8] C. Moore et al., Particle Accelerator Conference (PAC 03) 2003, Portland, Oregon, 2003.

[9] Addendum to the MiniBooNE Run Plan: MiniBooNE Physics in 2006 (2006), http://www-boone.fnal.gov/ publicpages/loi.ps.gz.

[10] M. Sorel, FERMILAB Report No. FERMILAB-THESIS2005-07, 2005.

[11] R. H. Nelson, AIP Conf. Proc. 842, 831 (2006).

[12] S. Agostinelli et al. (GEANT4), Nucl. Instrum. Methods Phys. Res., Sect. A 506, 250 (2003).

[13] The CERN Program Library (CERNLIB), see cernlib. web.cern.ch/cernlib.

[14] D. C. Carey, K. L. Brown, and F. Rothacker, SLAC Report No SLAC-R-0530, 1998.

[15] L. M. Chounet, J. M. Gaillard, and M. K. Gaillard, Phys. Rep. 4199 (1972).

[16] K. Hagiwara et al. (Particle Data Group), Phys. Rev. D 66, 010001 (2002).

[17] T. K. Gaisser, Cosmic Rays and Particle Physics (Univ. Pr., Cambridge, UK, 1990), p. 279.

[18] R. J. Glauber, in Lectures in Theoretical Physics, edited by W. E. Britten et al. (1959), Vol. 1.

[19] V. Franco, Phys. Rev. C 6, 748 (1972).

[20] R.D. Woods and D.S. Saxon, Phys. Rev. 95, 577 (1954).

[21] S. Eidelman et al. (Particle Data Group), Phys. Lett. B 592, 1 (2004).

[22] D. Harting et al., Nuovo Cimento 38, 60 (1965).

[23] K. J. Foley, R. S. Gilmore, S. J. Lindenbaum, W. A. Love, S. Ozaki, E.H. Willen, R. Yamada, and L.C.L. Yuan, Phys. Rev. Lett. 15, 45 (1965).

[24] K. J. Foley, S. J. Lindenbaum, W. A. Love, S. Ozaki, J. J. Russell, and L.C. L. Yuan, Phys. Rev. Lett. 11, 425
(1963).

[25] R. Clyde, Thesis, UCRL 16275.

[26] S. Colleti et al., Nuovo Cimento A 49, 479 (1967).

[27] G. Alexander, O. Benary, G. Czapek, B. Haber, N. Kidron, B. Reuter, A. Shapira, E. Simopoulou, and G. Yekutieli, Phys. Rev. 154, 1284 (1967).

[28] M. L. Perl, J. Cox, M. J. Longo, and M. N. Kreisler, Phys. Rev. D 1, 1857 (1970).

[29] B. G. Gibbard, L. W. Jones, M. J. Longo, J. R. O'Fallon, J. Cox, M. L. Perl, W.T. Toner, and M. N. Kreisler, Phys. Rev. Lett. 24, 22 (1970).

[30] C. T. Coffin, N. Dikmen, L. Ettlinger, D. Meyer, A. Saulys, K. Terwilliger, and D. Williams, Phys. Rev. 159, 1169 (1967).

[31] T. Lasinski, R. Levi Setti, B. Schwarzschild, and P. Ukleja, Nucl. Phys. B37, 1 (1971).

[32] A. S. Carroll et al., Phys. Rev. C 14, 635 (1976).

[33] V. V. Gachurin et al., Report No. ITEP-59-1985, 1985.

[34] B. M. Bobchenko et al., Sov. J. Nucl. Phys. 30, 805 (1979).

[35] D. Ashery et al., Phys. Rev. C 23, 2173 (1981).

[36] B. W. Allardyce et al., Nucl. Phys. A209, 1 (1973).

[37] R. M. Barnett et al. (Particle Data Group), Phys. Rev. D 54, 1 (1996).

[38] M. G. Catanesi et al., Eur. Phys. J. C 52, 29 (2007), (Note: The HARP data used in the MiniBooNE analysis result from a preliminary analysis of the data prior to publication. The cross sections therefore differ slightly from those reported in this publication.), arXiv:hep-ex/0702024.

[39] I. Chemakin et al. (E910), Phys. Rev. C 77, 015209 (2008).

[40] Y. Cho, M. Derrick, G. Marmer, T. P. Wangler, J. L. Day, P. Kalbaci, M. L. Marshak, J. K. Randolph, and A. W. Key, Phys. Rev. D 4, 1967 (1971).

[41] I. A. Vorontsov et al., Report No. ITEP-83-085, 1983.

[42] J. Allaby, A. Diddens, R. Glauber, A. Klovning, O. Kofoed-Hansen, E. J. Sacharidis, K. Schlpmann, A. M. Thorndike, and A. M. Wetherell, Phys. Lett. B 30, 549 (1969).

[43] G. J. Marmer and D. E. Lundquist, Phys. Rev. D 3, 1089 (1971).

[44] Y. D. Aleshin, I. A. Drabkin, and V. V. Kolesnikov, Report No. ITEP-80-1977, 1977.

[45] M. H. Ahn et al. (K2K), Phys. Rev. D 74, 072003 (2006).

[46] J. R. Sanford and C. L. Wang, BNL Note 11299, 1967.

[47] HARP Collaboration (unpublished).

[48] T. Abbott, Y. Akiba, D. Beavis, M. A. Bloomer, P. D. Bond, C. Chasman, Z. Chen, Y. Y. Chu, B. A. Cole, J. B. 
Costales et al., Phys. Rev. D 45, 3906 (1992).

[49] J. V. Allaby et al., CERN Report No. CERN 70-12 (unpublished).

[50] D. Dekkers, J. A. Geibel, R. Mermod, G. Weber, T. R. Willitts, K. Winter, B. Jordan, M. Vivargent, N. M. King, and E. J. N. Wilson, Phys. Rev. 137, B962 (1965).

[51] T. Eichten et al., Nucl. Phys. B44, 333 (1972).

[52] R. A. Lundy, T. B. Novey, D. D. Yovanovitch, and V.L. Telegdi, Phys. Rev. Lett. 14, 504 (1965).

[53] G. J. Marmer, K. Reibel, D. M. Schwartz, A. Stevens, R. Winston, D. Wolfe, C.J. Rush, P. R. Phillips, E.C. Swallow, and T. A. Romanowski, Phys. Rev. 179, 1294 (1969).

[54] I. A. Vorontsov, G. A. Safronov, A. A. Sibirtsev, G. N. Smirnov, and Y. V. Trebukhovsky, Report No. ITEP-88011, 1988.

[55] J. M. Conrad, J. R. Monroe, and M.H. Shaevitz (unpublished).
[56] F. Abe et al., Phys. Rev. D 36, 1302 (1987).

[57] N. V. Mokhov et al., arXiv:nucl-th/9812038.

[58] A. Ferrari, P. R. Sala, A. Fasso, and J. Ranft, CERN Report No. CERN-2005-010, FLUKA: A multiparticle transport code (Program version 2005).

[59] G. Bellettini et al., Nucl. Phys. B79, 609 (1966).

[60] D. Casper, Nucl. Phys. B, Proc. Suppl. 112, 161 (2002).

[61] A.A. Aguilar-Arevalo et al. (MiniBooNE), Nucl. Instrum. Methods Phys. Res., Sect. A 599, 28 (2009).

[62] W.F. Schimmerling et al., Phys. Lett. B 37, 177 (1971).

[63] T. Coor, D. A. Hill, W. F. Hornyak, L. W. Smith, and G. Snow, Phys. Rev. 98, 1369 (1955).

[64] W. L. Lakin et al., Phys. Lett. B 31, 677 (1970).

[65] E. F. Parker et al., Phys. Lett. B 31, 246 (1970).

[66] J. Engler et al., Phys. Lett. B 27, 599 (1968). 